Supporting Information

\title{
Electrophilic Substitution of Asymmetrically Distorted Benzenes within Triptycene Derivatives
}

Keisuke Ueno, ${ }^{\dagger}$ Yuji Nishii, ${ }^{*}{ }^{\dagger}$ and Masahiro Miura*,†

${ }^{\dagger}$ Department of Applied Chemistry, Graduate School of Engineering, Osaka University, Suita, Osaka 565-0871, Japan

y-nishii@chem.eng.osaka-u.ac.jp

miura@chem.eng.osaka-u.ac.jp

\section{Contents}

1. General Information $\quad$ S2

2. Experimental Procedure and Identification Data S3

3. Additional Data $\quad \mathrm{S} 10$

4. X-Ray Crystallographic Analysis $\quad \mathrm{S} 16$

5. Computational Study $\quad \mathrm{S} 18$

6. Copy of NMR Spectra $\quad$ S21

7. References $\quad$ S58 


\section{General Information}

All manipulations were performed under $\mathrm{N}_{2}$ using standard Schlenk techniques unless otherwise noted. Toluene and $\mathrm{MeCN}$ were dried and deoxygenated by a Glass Counter Solvent Dispending System (Nikko Hansen \& Co., Ltd.). 1,2-Dichloroethane (DCE) and 1,4-dioxane were distilled from $\mathrm{CaH}_{2}$ and stored under $\mathrm{N}_{2}$ with molecular sieves $4 \mathrm{~A}$. THF was purchased as dehydrated solvent and used as received. Silica gel column chromatography was performed using Wakosil ${ }^{\circledR}$ C-200 $(64-210 \mu \mathrm{m})$. Trip-SMe (1b) ${ }^{1}$ and Trip-OMe (1c) ${ }^{2}$ were prepared according to the literature. $N$-Nitrosaccharin was prepared according to the literature. ${ }^{3}$

Nuclear magnetic resonance spectra were measured at $400 \mathrm{MHz}\left({ }^{1} \mathrm{H} \mathrm{NMR}\right)$, at $100 \mathrm{MHz}\left({ }^{13} \mathrm{C}\right.$ $\mathrm{NMR})$, and at $376 \mathrm{MHz}\left({ }^{19} \mathrm{~F} \mathrm{NMR}\right)$ in $5 \mathrm{~mm}$ NMR tubes. ${ }^{1} \mathrm{H}$ NMR chemical shifts were reported in ppm relative to the resonance of TMS $(\delta 0.00)$ or the residual solvent signals at $\delta 7.26$ for $\mathrm{CDCl}_{3}$. ${ }^{13} \mathrm{C}$ NMR chemical shifts were reported in ppm relative to the residual solvent signals at $\delta 77.2$ for $\mathrm{CDCl}_{3}$. Melting points were measured with Mettler Toledo MP90. High resolution mass spectra (HRMS) were recorded by APCI-TOF or EI-TOF. Single crystal X-ray diffraction analyses were conducted with Rigaku XtaLAB Synergy diffractometer and HyPix-6000 detector. Preparative gel permeation chromatography (GPC) was conducted with Showa Denko H-2001, H-2002 column (eluent: $\mathrm{CHCl}_{3}$ ) column. 


\section{Experimental Procedures and Identification Data}

\section{2-1. Preparation of Trip-TMS (1e)}

To a two-neck round-bottom flask equipped with an $\mathrm{N}_{2}$ balloon were added 9-bromotriptycene $(1.67 \mathrm{~g}, 5.0 \mathrm{mmol})$ and THF $(25 \mathrm{~mL})$. The solution was cooled to $-78{ }^{\circ} \mathrm{C}$ and $n-\mathrm{BuLi}(1.57 \mathrm{~mol} / \mathrm{L}$ in $n$-hexane, $6.0 \mathrm{mmol}$ ) was added dropwise, and the resulting suspension was stirred for $1 \mathrm{~h}$ at this temperature. Chlorotrimethylsilane $(0.76 \mathrm{~mL}, 6.0 \mathrm{mmol})$ was added via syringe. The mixture was allowed to warm to room temperature with continuous stirring over $2 \mathrm{~h}$. The resulting solution was poured into aqueous $\mathrm{NH}_{4} \mathrm{Cl}$ solution and extracted with $\mathrm{Et}_{2} \mathrm{O}$ three times. The combined organic layers was dried over $\mathrm{Na}_{2} \mathrm{SO}_{4}$, concentrated in vacuo, and purified by silica gel chromatography (hexane/EtOAc $=10 / 1)$ to give Trip-TMS as white solid (1.60 g, 98\% yield).

9-(trimethylsilyl)triptycene (1e)

m.p. $>300{ }^{\circ} \mathrm{C} ;{ }^{1} \mathrm{H}$ NMR $\left(400 \mathrm{MHz}, \mathrm{CDCl}_{3}\right) \delta$ 7.46-7.44 (m, 3H), 7.39-7.37 (m, 3H), 7.00-6.93 (m, $6 \mathrm{H}), 5.33(\mathrm{~s}, 1 \mathrm{H}), 0.76(\mathrm{~s}, 9 \mathrm{H}) ;{ }^{13} \mathrm{C} \mathrm{NMR}\left(100 \mathrm{MHz}, \mathrm{CDCl}_{3}\right) \delta 148.5,146.6,125.3,124.8,124.5$, 124.1, 55.3, 47.8, 2.0; HRMS (APCI) $m / z$ : $[\mathrm{M}+\mathrm{H}]^{+}$Calcd for $\mathrm{C}_{23} \mathrm{H}_{23} \mathrm{Si} 327.1564$; Found 327.1568.

\section{2-2. Preparation of Trip-t-Bu (1f)}

9-t-Butylanthracene was prepared according to the literature. ${ }^{4}$ To a two-neck round-bottom flask equipped with an $\mathrm{N}_{2}$ balloon was added $t$ - $\mathrm{BuMgCl}(1.0 \mathrm{~mol} / \mathrm{L}$ in THF, $30 \mathrm{mmol})$. Anthrone (1.94 $\mathrm{g}, 10 \mathrm{mmol})$ was added to the flask dropwise as a THF solution $(24 \mathrm{~mL})$ at $0{ }^{\circ} \mathrm{C}$. The mixture was allowed to warm to room temperature with continuous stirring over $1 \mathrm{~h}$. The resulting solution was poured into aqueous $\mathrm{NH}_{4} \mathrm{Cl}$ solution and extracted with EtOAc three times. The combined organic layers was dried over $\mathrm{Na}_{2} \mathrm{SO}_{4}$, concentrated in vacuo, and purified by silica gel chromatography (hexane/EtOAc $=20 / 1)$ to give 9-t-butyl-9,10-dihydroanthracen-9-ol (472 mg, 25\% yield).

The obtained alcohol $(252.7 \mathrm{mg}, 1.0 \mathrm{mmol}), \mathrm{P}_{2} \mathrm{O}_{5}(250 \mathrm{mg})$, and $\mathrm{CCl}_{4}(4.0 \mathrm{~mL})$ were placed in a round-bottom flask. The suspension was stirred at room temperature for $6 \mathrm{~h}$. The resulting mixture was poured into aqueous $\mathrm{NH}_{4} \mathrm{Cl}$ solution and extracted with $\mathrm{CHCl}_{3}$ three times. The combined organic layers was dried over $\mathrm{Na}_{2} \mathrm{SO}_{4}$, concentrated in vacuo, and purified by silica gel chromatography (hexane $/ \mathrm{CHCl}_{3}=1 / 1$ ) to give 9-t-butylanthracene $(163.4 \mathrm{mg}, 70 \%$ yield).

To a two-neck round-bottom flask equipped with an $\mathrm{N}_{2}$ balloon were added 9-t-butylanthracene (140.6 mg, $0.6 \mathrm{mmol})$ and $\mathrm{CsF}$ (182.3 mg, $2.0 \mathrm{mmol}) . \mathrm{MeCN}$ (4.0 mL) and 2(trimethylsilyl)phenyl triflate $(291 \mu \mathrm{L}, 2.0 \mathrm{mmol})$ were added to the flask via syringe. The mixture 
was heated at $70{ }^{\circ} \mathrm{C}$ with an oil bath for $23 \mathrm{~h}$. The resulting solution was poured into aqueous $\mathrm{NH}_{4} \mathrm{Cl}$ solution and extracted with $\mathrm{CHCl}_{3}$ three times. The combined organic layers was dried over $\mathrm{Na}_{2} \mathrm{SO}_{4}$, concentrated in vacuo, and purified by $\mathrm{GPC}\left(\mathrm{CHCl}_{3}\right)$ to Trip- $t$ - $\mathrm{Bu}(98.1 \mathrm{mg}, 86 \%$ yield).

9-t-butyltriptycene (1f $)^{5}$

white solid; m.p. 188.6-190.6 ${ }^{\circ} \mathrm{C} ;{ }^{1} \mathrm{H}$ NMR $\left(400 \mathrm{MHz}, \mathrm{CDCl}_{3}\right) \delta$ 7.81-7.77 (m, 3H), 7.38-7.34 (m, 3H), 6.98-6.94 (m, 6H), $5.23(\mathrm{~s}, 1 \mathrm{H}), 2.09(\mathrm{~s}, 9 \mathrm{H}) ;{ }^{13} \mathrm{C} \mathrm{NMR}\left(100 \mathrm{MHz}, \mathrm{CDCl}_{3}\right) \delta 148.5,144.8$, 126.8, 124.7, 123.9, 123.9, 65.9, 56.1, 33.7, 33.0; HRMS (APCI) $m / z:[\mathrm{M}+\mathrm{H}]^{+}$Calcd for $\mathrm{C}_{24} \mathrm{H}_{23}$ 311.1794; Found 311.1796.

\section{2-3. General Procedure for the Bromination (Table 1)}

To an oven-dried Schlenk tube were added Trip-R (1, $0.2 \mathrm{mmol})$, Trip-SMe (0.6 mg, $1.0 \mathrm{~mol} \%)$, NBS (128.1 mg, $0.72 \mathrm{mmol})$, and $\mathrm{AgSbF}_{6}(0.7 \mathrm{mg}, 1.0 \mathrm{~mol} \%)$. DCE (2.0 mL) was added via syringe, and the mixture was stirred at room temperature for $15 \mathrm{~h}$. The resulting solution was poured into aqueous $\mathrm{NaHCO}_{3}$ solution and extracted with $\mathrm{CHCl}_{3}$ three times. The combined organic layers was dried over $\mathrm{Na}_{2} \mathrm{SO}_{4}$, concentrated in vacuo, and purified by silica gel chromatography to give the corresponding product. Single crystals of $\mathbf{2 e}$ and $\mathbf{2 f}$ suitable for X-ray analysis were obtained by $n$-pentane vapor diffusion into the $\mathrm{CHCl}_{3}$ solution.

2,7,14-tribromo-9-(trimethylsilyl)triptycene (2e)
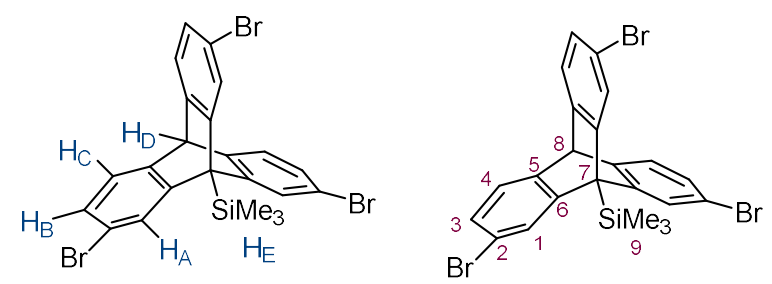

Purified by silica gel chromatography (hexane/EtOAc $=20 / 1)$ as white solid $(68.7 \mathrm{mg}, 61 \%$ yield); m.p. 211.9-213.9 ${ }^{\circ} \mathrm{C} ;{ }^{1} \mathrm{H}$ NMR $\left(400 \mathrm{MHz}, \mathrm{CDCl}_{3}\right) \delta 7.55\left(\mathrm{H}_{\mathrm{A}}, \mathrm{d}, J=1.7 \mathrm{~Hz}, 3 \mathrm{H}\right), 7.24\left(\mathrm{H}_{\mathrm{C}}, \mathrm{d}, J=\right.$ $7.9 \mathrm{~Hz}, 3 \mathrm{H}), 7.15\left(\mathrm{H}_{\mathrm{B}}, \mathrm{dd}, J=7.9,1.7 \mathrm{~Hz}, 3 \mathrm{H}\right), 5.24\left(\mathrm{H}_{\mathrm{D}}, \mathrm{s}, 1 \mathrm{H}\right), 0.78\left(\mathrm{H}_{\mathrm{E}}, \mathrm{s}, 9 \mathrm{H}\right) ;{ }^{13} \mathrm{C} \mathrm{NMR}(100$ $\mathrm{MHz}, \mathrm{CDCl}_{3}$ ) $\delta 147.8(\mathrm{C} 6), 146.7(\mathrm{C} 5), 129.0(\mathrm{C} 1), 128.2(\mathrm{C} 3), 125.5(\mathrm{C} 4), 118.6(\mathrm{C} 2), 53.5$ (C8), 47.5 (C7), 1.6 (C9); HRMS (APCI) $m / z$ : $[\mathrm{M}+\mathrm{H}]^{+}$Calcd for $\mathrm{C}_{23} \mathrm{H}_{20}{ }^{79} \mathrm{Br}^{81} \mathrm{Br}_{2} \mathrm{Si}$ 564.8839; Found 564.8820 . 
2,7,14-tribromo-9-t-butyltriptycene (2f)
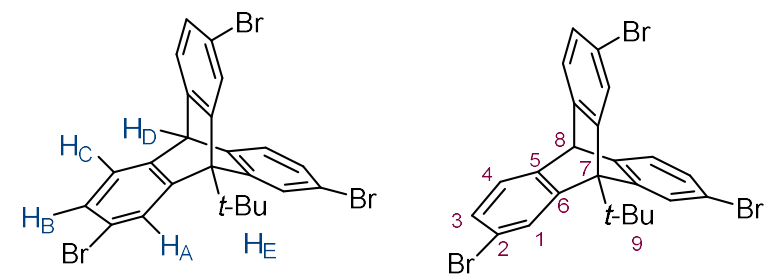

Purified by silica gel chromatography (hexane/EtOAc $=20 / 1)$ as white solid $(27.9 \mathrm{mg}, 51 \%$ yield); m.p. $>300{ }^{\circ} \mathrm{C} ;{ }^{1} \mathrm{H} \mathrm{NMR}\left(400 \mathrm{MHz}, \mathrm{CDCl}_{3}\right) \delta 7.91\left(\mathrm{H}_{\mathrm{A}}, \mathrm{d}, J=1.7 \mathrm{~Hz}, 3 \mathrm{H}\right), 7.21\left(\mathrm{H}_{\mathrm{C}}, \mathrm{d}, J=7.8 \mathrm{~Hz}\right.$, $3 \mathrm{H}), 7.15\left(\mathrm{H}_{\mathrm{B}}, \mathrm{dd}, J=7.8,1.7 \mathrm{~Hz}, 3 \mathrm{H}\right), 5.14\left(\mathrm{H}_{\mathrm{D}}, \mathrm{s}, 1 \mathrm{H}\right), 2.06\left(\mathrm{H}_{\mathrm{E}}, \mathrm{s}, 9 \mathrm{H}\right) ;{ }^{13} \mathrm{C} \mathrm{NMR}(100 \mathrm{MHz}$, $\mathrm{CDCl}_{3}$ ) $\delta 146.6(\mathrm{C} 5), 145.9(\mathrm{C} 6), 130.4(\mathrm{C} 1), 128.1$ (C3), 125.2 (C4), 118.2 (C2), 66.0 (C7), 54.2 (C8), 33.4 (C9), 32.8 (C10); HRMS (APCI) $m / z$ : [M+H] $]^{+}$Calcd for $\mathrm{C}_{24} \mathrm{H}_{20}{ }^{79} \mathrm{Br}^{81} \mathrm{Br}_{2}$ 547.8991; Found 547.8973 .

\section{2-4. TMS Group Removal of $2 \mathrm{e}$ (Scheme 2)}

To a screw-top tube was added $2 \mathrm{e}(56.3 \mathrm{mg}, 0.1 \mathrm{mmol})$ and filled with $\mathrm{N}_{2}$. THF $(2.0 \mathrm{~mL})$ and TBAF $(1.0 \mathrm{~mol} / \mathrm{L}$ in THF, $0.15 \mathrm{mmol})$ were added via syringe. The mixture heated at $80{ }^{\circ} \mathrm{C}$ with an oil bath for $18 \mathrm{~h}$. The resulting solution was poured into water, extracted with $\mathrm{CHCl}_{3}$, and dried over $\mathrm{Na}_{2} \mathrm{SO}_{4}$. Volatiles were removed in vacuo to give 2,7,14-tribromotriptycene (4) as white solid (48.8 mg, 99\% yield).

2,7,14-tribromotriptycene $(4)^{6}$

m.p. $>300{ }^{\circ} \mathrm{C}$; ${ }^{1} \mathrm{H}$ NMR $\left(400 \mathrm{MHz}, \mathrm{CDCl}_{3}\right) \delta 7.51(\mathrm{~d}, J=1.7 \mathrm{~Hz}, 3 \mathrm{H}), 7.23(\mathrm{~d}, J=7.8 \mathrm{~Hz}, 3 \mathrm{H})$, $7.15(\mathrm{dd}, J=7.8,1.7 \mathrm{~Hz}, 3 \mathrm{H}), 5.33(\mathrm{~s}, 1 \mathrm{H}), 5.28(\mathrm{~s}, 1 \mathrm{H}) ;{ }^{13} \mathrm{C} \mathrm{NMR}\left(100 \mathrm{MHz}, \mathrm{CDCl}_{3}\right) \delta 146.2$, 143.6, 128.6, 127.4, 125.3, 119.2, 53.0, 52.5; HRMS (APCI) $m / z$ : $[\mathrm{M}+\mathrm{H}]^{+}$Calcd for $\mathrm{C}_{20} \mathrm{H}_{11} \mathrm{Br}_{3}$ 492.8443; Found 492.8448.

\section{2-5. Scale-Up for the Bromination of 1e (Scheme 2)}

To an oven-dried Schlenk tube were added Trip-TMS (1e) (653 mg, $2.0 \mathrm{mmol})$, Trip-SMe (6.0 mg, $1.0 \mathrm{~mol} \%)$, NBS (1.1 g, $6.1 \mathrm{mmol})$, and $\mathrm{AgSbF}_{6}(6.9 \mathrm{mg}, 1.0 \mathrm{~mol} \%)$. DCE (25 mL) was added via syringe, and the mixture was stirred at room temperature for $15 \mathrm{~h}$. The resulting solution was poured into aqueous $\mathrm{NaHCO}_{3}$ solution and extracted with $\mathrm{CHCl}_{3}$ three times. The combined organic layers was dried over $\mathrm{Na}_{2} \mathrm{SO}_{4}$, filtered through a pad of silica gel, and concentrated in vacuo. The obtained crude material was purified by recrystallization ( $n$-pentane vapor diffusion into the $\mathrm{CHCl}_{3}$ solution) to give analytically pure $2 \mathrm{e}(562 \mathrm{mg}, 50 \%$ yield $)$. 


\section{2-6. Synthesis of 5 via Sonogashira Coupling (Scheme 3)}

To a two-neck round-bottom flask equipped with an $\mathrm{N}_{2}$ balloon were added 4 ( $24.6 \mathrm{mg}, 0.05 \mathrm{mmol}$ ), $\mathrm{CuI}(1.0 \mathrm{mg}, 10 \mathrm{~mol} \%), \mathrm{Pd}\left(\mathrm{PPh}_{3}\right)_{4}(8.7 \mathrm{mg}, 15 \mathrm{~mol} \%)$, and toluene $(2.0 \mathrm{~mL}) . i-\mathrm{Pr}_{2} \mathrm{NH}(53 \mu \mathrm{L}$, $0.38 \mathrm{mmol})$ and trimethylsilylacetylene $(51.9 \mu \mathrm{L}, 0.38 \mathrm{mmol})$ were added dropwise via syringe, and the mixture was heated at $80^{\circ} \mathrm{C}$ with an oil bath for $18 \mathrm{~h}$. The resulting suspension was filtered through a pad of alumina eluting with $\mathrm{CHCl}_{3}$. The filtrate was dried over $\mathrm{Na}_{2} \mathrm{SO}_{4}$, concentrated in vacuo, and purified by silica gel chromatography (hexane/EtOAc $=40 / 1$ to 20/1) to give 5 as white solid (21.3 mg, 78\% yield).

2,7,14-((trimethylsilyl)ethynyl)triptycene $(\mathbf{5})^{7}$

white solid; m.p. 136.6-138.6 ${ }^{\circ} \mathrm{C} ;{ }^{1} \mathrm{H}$ NMR $\left(400 \mathrm{MHz}, \mathrm{CDCl}_{3}\right) \delta 7.44(\mathrm{~d}, J=1.4 \mathrm{~Hz}, 3 \mathrm{H}), 7.27$ (d, $J=8.0 \mathrm{~Hz}, 3 \mathrm{H}), 7.12(\mathrm{dd}, J=8.0,1.4 \mathrm{~Hz}, 3 \mathrm{H}), 5.35(\mathrm{~s}, 1 \mathrm{H}), 5.28(\mathrm{~s}, 1 \mathrm{H}), 0.21(\mathrm{~s}, 27 \mathrm{H}) ;{ }^{13} \mathrm{C} \mathrm{NMR}$ $\left(100 \mathrm{MHz}, \mathrm{CDCl}_{3}\right) \delta 144.7,144.5,129.5,127.3,123.7,120.4,105.2,93.6,53.7,53.3,0.1 ;$ HRMS (APCI) $m / z:[\mathrm{M}+\mathrm{H}]^{+}$Calcd for $\mathrm{C}_{35} \mathrm{H}_{39} \mathrm{Si}_{3}$ 543.2354; Found 543.2360.

\section{2-7. Synthesis of 6 via Buchwald-Hartwig Coupling (Scheme 3)}

To a two-neck round-bottom flask equipped with an $\mathrm{N}_{2}$ balloon were added 4 (49.1 mg, $0.1 \mathrm{mmol}$ ), $\mathrm{Pd}(\mathrm{OAc})_{2}(3.7 \mathrm{mg}, 15 \mathrm{~mol} \%), t$-BuXPhos $(8.5 \mathrm{mg}, 20 \mathrm{~mol} \%), \mathrm{K}_{3} \mathrm{PO}_{4}(127.4 \mathrm{mg}, 0.6 \mathrm{mmol})$, phenol $(33.9 \mathrm{mg}, 0.36 \mathrm{mmol})$, and toluene $(2.0 \mathrm{~mL})$. The mixture was heated at $100{ }^{\circ} \mathrm{C}$ with an oil bath for $24 \mathrm{~h}$. The resulting suspension was filtered through a pad of alumina eluting with $\mathrm{CHCl}_{3}$. The filtrate was dried over $\mathrm{Na}_{2} \mathrm{SO}_{4}$, concentrated in vacuo, and purified by silica gel chromatography (hexane/EtOAc $=20 / 1$ to $10 / 1)$ to give 6 as white solid $(22.3 \mathrm{mg}, 42 \%$ yield).

2,7,14-triphenyloxytriptycene (6)

m.p. 246.7-248.7 ${ }^{\circ} \mathrm{C} ;{ }^{1} \mathrm{H}$ NMR $\left(400 \mathrm{MHz}, \mathrm{CDCl}_{3}\right) \delta$ 7.32-7.28 (m, 9H), $7.08(\mathrm{tt}, J=7.3,1.1 \mathrm{~Hz}$, $3 \mathrm{H}), 7.02(\mathrm{~d}, J=2.3 \mathrm{~Hz}, 3 \mathrm{H}), 6.95(\mathrm{dt}, J=7.6,1.0 \mathrm{~Hz}, 6 \mathrm{H}), 6.64(\mathrm{dd}, J=8.1,2.3 \mathrm{~Hz}, 3 \mathrm{H}), 5.36$ (s, $1 \mathrm{H}), 5.18(\mathrm{~s}, 1 \mathrm{H}) ;{ }^{13} \mathrm{C} \mathrm{NMR}\left(100 \mathrm{MHz}, \mathrm{CDCl}_{3}\right) \delta 157.4,154.8,146.7,140.6,129.8,124.3$, 123.3, 119.1, 115.1, 54.3, 52.2 (1 peak overlapped); HRMS (APCI) $\mathrm{m} / z$ : $[\mathrm{M}+\mathrm{H}]^{+}$Calcd for $\mathrm{C}_{38} \mathrm{H}_{27} \mathrm{O}_{3}$ 531.1955; Found 531.1939.

\section{2-8. Synthesis of 7 via Buchwald-Hartwig Coupling (Scheme 3)}

To a two-neck round-bottom flask equipped with an $\mathrm{N}_{2}$ balloon were added 4 (49.1 mg, $0.1 \mathrm{mmol}$ ), $\mathrm{Pd}(\mathrm{OAc})_{2}(3.7 \mathrm{mg}, 15 \mathrm{~mol} \%)$, dppf (24.9 mg, $\left.45 \mathrm{~mol} \%\right), t$-BuONa (86.5 mg, $\left.0.9 \mathrm{mmol}\right),(p-$ 
$\left.\mathrm{MeC}_{6} \mathrm{H}_{4}\right)_{2} \mathrm{NH}(118.4 \mathrm{mg}, 0.6 \mathrm{mmol})$, and toluene $(2.0 \mathrm{~mL})$. The mixture was heated at $100{ }^{\circ} \mathrm{C}$ with an oil bath for $18 \mathrm{~h}$. The resulting suspension was filtered through a pad of alumina eluting with $\mathrm{CHCl}_{3}$. The filtrate was dried over $\mathrm{Na}_{2} \mathrm{SO}_{4}$, concentrated in vacuo, and purified by silica gel chromatography (hexane/EtOAc $=20 / 1)$ and $\mathrm{GPC}\left(\mathrm{CHCl}_{3}\right)$ to give 7 as grayish white solid (44.4 $\mathrm{mg}, 78 \%$ yield).

2,7,14-tris(di-p-tolylamino)triptycene (7)

m.p. $>300{ }^{\circ} \mathrm{C} ;{ }^{1} \mathrm{H}$ NMR $\left(400 \mathrm{MHz}, \mathrm{CDCl}_{3}\right) \delta 7.13(\mathrm{~d}, J=8.0 \mathrm{~Hz}, 3 \mathrm{H}), 7.00(\mathrm{~d}, J=8.2 \mathrm{~Hz}, 12 \mathrm{H})$, $6.95(\mathrm{~d}, J=2.1 \mathrm{~Hz}, 3 \mathrm{H}), 6.90(\mathrm{~d}, J=8.4 \mathrm{~Hz}, 12 \mathrm{H}), 6.64(\mathrm{dd}, J=8.0,2.1 \mathrm{~Hz}, 3 \mathrm{H}), 5.17(\mathrm{~s}, 1 \mathrm{H})$, 4.85 (s, 1H), 2.28 (s, 18H); $\left.{ }^{13} \mathrm{C} \mathrm{NMR} \mathrm{(100} \mathrm{MHz,} \mathrm{CDCl}_{3}\right) \delta 146.3,145.7,145.3,139.7,132.1,129.9$, 124.5, 123.6, 119.6, 119.2, 54.3, 52.3, 20.9; HRMS (APCI) $m / z:[\mathrm{M}+\mathrm{H}]^{+}$Calcd for $\mathrm{C}_{62} \mathrm{H}_{54} \mathrm{~N}_{3}$ 840.4312 ; Found 840.4305 .

\section{2-9. Synthesis of 8 via Direct Borylation (Scheme 3)}

To a two-neck round-bottom flask equipped with an $\mathrm{N}_{2}$ balloon were added 4 ( $24.5 \mathrm{mg}, 0.05 \mathrm{mmol}$ ), $\mathrm{PdCl}_{2}\left(\mathrm{PPh}_{3}\right)_{2}\left(3.5 \mathrm{mg}, 10 \mathrm{~mol} \%\right.$ ), B $\mathrm{pin}_{2}$ (41.9 mg, $\left.0.17 \mathrm{mmol}\right), \mathrm{KOAc}(16.2 \mathrm{mg}, 0.17 \mathrm{mmol})$, and 1,4-dioxane $(2.0 \mathrm{~mL})$. The mixture was heated at $100{ }^{\circ} \mathrm{C}$ with an oil bath for $18 \mathrm{~h}$. The resulting suspension was filtered through a pad of alumina eluting with $\mathrm{CHCl}_{3}$. The filtrate was dried over $\mathrm{Na}_{2} \mathrm{SO}_{4}$, concentrated in vacuo, and purified by silica gel chromatography (hexane/EtOAc $=20 / 1$ ) and $\mathrm{GPC}\left(\mathrm{CHCl}_{3}\right)$ to give 8 as white solid $(22.6 \mathrm{mg}, 71 \%$ yield).

\section{2,7,14-tris(pinacolatoboryl)triptycene (8)}

m.p. 292.6-294.6 ${ }^{\circ} \mathrm{C} ;{ }^{1} \mathrm{H} \mathrm{NMR}\left(400 \mathrm{MHz}, \mathrm{CDCl}_{3}\right) \delta 7.78(\mathrm{~s}, 3 \mathrm{H}), 7.44(\mathrm{dd}, J=7.3,1.0 \mathrm{~Hz}, 3 \mathrm{H})$, $7.37(\mathrm{~d}, J=7.3 \mathrm{~Hz}, 3 \mathrm{H}), 5.48(\mathrm{~s}, 1 \mathrm{H}), 5.44(\mathrm{~s}, 1 \mathrm{H}), 1.28(\mathrm{~s}, 36 \mathrm{H}) ;{ }^{13} \mathrm{C} \mathrm{NMR}\left(100 \mathrm{MHz}, \mathrm{CDCl}_{3}\right) \delta$ $147.8,144.6,132.4,129.7,123.4,83.7,54.8,53.9,24.9$ (carbon atoms directly bonded to boron atoms were not observed); HRMS (EI) $m / z\left([M]^{+}\right.$) calcd for $\mathrm{C}_{38} \mathrm{H}_{47} \mathrm{~B}_{3} \mathrm{O}_{6}: 632.3665$, found: 632.3656 .

\section{2-10. Synthesis of 9 (Scheme 3)}

To a two-neck round-bottom flask equipped with an $\mathrm{N}_{2}$ balloon were added $\mathbf{8}$ (31.6 $\mathrm{mg}, 0.05 \mathrm{mmol}$ ), $\mathrm{PdCl}_{2}\left(\mathrm{PPh}_{3}\right)_{2}$ (3.5 mg, $10 \mathrm{~mol} \%$ ), and tetrabutylammonium bromide (1.6 mg, $10 \mathrm{~mol} \%$ ). 1 Bromo-2-(methylsulfinyl)benzene $(42.7 \mathrm{md}, 0.2 \mathrm{mmol}$ ) was added to the flask as THF solution $\left(2.0 \mathrm{~mL}\right.$ ), and aqueous $\mathrm{K}_{2} \mathrm{CO}_{3}$ solution (degassed by $\mathrm{N}_{2}$ bubbling, $2.0 \mathrm{~mol} / \mathrm{L}, 0.13 \mathrm{~mL}$ ) was added 
dropwise via syringe. The mixture was heated at $80{ }^{\circ} \mathrm{C}$ with an oil bath for $24 \mathrm{~h}$. The resulting suspension was filtered through a pad of alumina eluting with $\mathrm{CHCl}_{3}$. The filtrate was dried over $\mathrm{Na}_{2} \mathrm{SO}_{4}$, concentrated in vacuo, and subjected to $\mathrm{GPC}\left(\mathrm{CHCl}_{3}\right)$ to give the crude coupling product, which used for the next step without further purification.

The obtained crude material was dissolved in DCE (5.0 mL), and TfOH (360 mg, $2.4 \mathrm{mmol})$ was added dropwise. The mixture was stirred at room temperature for $24 \mathrm{~h}$. The resulting solution was poured into aqueous pyridine solution (pyridine $2.0 \mathrm{~mL}+\mathrm{H}_{2} \mathrm{O} 10 \mathrm{~mL}$ ), and the mixture was refluxed with an oil bath for $4 \mathrm{~h}$. The resulting suspension was poured into $\mathrm{HCl}(1.0 \mathrm{~mol} / \mathrm{L})$ and extracted with $\mathrm{CHCl}_{3}$ three times. The combined organic layers was dried over $\mathrm{Na}_{2} \mathrm{SO}_{4}$, filtered through a pad of silica gel, concentrated in vacuo, and purified by silica gel chromatography (hexane/EtOAc $=5 / 1)$ to give 9 as white solid $(4.6 \mathrm{mg}, 16 \%$ yield).

benzo $[b]$ thiophene-fused triptycene $(9)$

m.p. 269.9-271.9 ${ }^{\circ} \mathrm{C} ;{ }^{1} \mathrm{H}$ NMR $\left(400 \mathrm{MHz}, \mathrm{CDCl}_{3}\right) \delta 8.24(\mathrm{~s}, 3 \mathrm{H}), 8.09-8.07$ (m, 3H), $7.90(\mathrm{~s}, 3 \mathrm{H})$, 7.78-7.76 (m, 3H), 7.43-7.34 (m, 6H), $5.88(\mathrm{~s}, 1 \mathrm{H}), 5.69(\mathrm{~s}, 1 \mathrm{H}) ;{ }^{13} \mathrm{C} \mathrm{NMR}\left(100 \mathrm{MHz}, \mathrm{CDCl}_{3}\right) \delta$ 143.5, 142.0, 139.9, 137.0, 135.5, 133.1, 126.3, 124.4, 123.0, 121.3, 118.3, 116.8, 54.3, 54.2; HRMS (APCI) $m / z:[\mathrm{M}+\mathrm{H}]^{+}$Calcd for $\mathrm{C}_{38} \mathrm{H}_{21} \mathrm{~S}_{3}$ 573.0800; Found 573.0787.

\section{2-11. Trifluoromethylthiolation of 1e (Scheme 4)}

To an oven-dried Schlenk tube were added Trip-TMS (1e) $(65.3 \mathrm{mg}, 0.2 \mathrm{mmol})$ and $\mathrm{N}-\mathrm{SCF}_{3}$ saccharin (203.9 mg, $0.72 \mathrm{mmol})$. TfOH $(27 \mathrm{mg}, 0.18 \mathrm{mmol})$ in DCE $(5.0 \mathrm{~mL})$ was added to the tube via syringe, and the mixture was stirred at room temperature for $15 \mathrm{~h}$. The resulting solution was poured into aqueous $\mathrm{NaHCO}_{3}$ solution and extracted with $\mathrm{CHCl}_{3}$ three times. The combined organic layers was dried over $\mathrm{Na}_{2} \mathrm{SO}_{4}$, and concentrated in vacuo. The obtained crude material was subjected to the same reaction conditions again. After workup, the target product was isolated by silica gel chromatography (hexane/EtOAc $=20 / 1)$ to give $\mathbf{1 0}$ as white solid (42.3 $\mathrm{mg}, 35 \%$ yield).

2,7,14-tris(trifluoromethylthio)-9-(trimethylsilyl)triptycene (10)
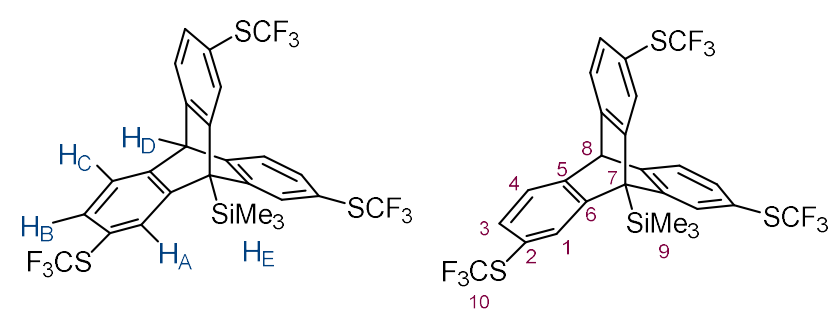
m.p. 231.6-233.6 ${ }^{\circ} \mathrm{C} ;{ }^{1} \mathrm{H}$ NMR $\left(400 \mathrm{MHz}, \mathrm{CDCl}_{3}\right) \delta 7.76\left(\mathrm{H}_{\mathrm{A}}, \mathrm{s}, 3 \mathrm{H}\right), 7.46\left(\mathrm{H}_{\mathrm{C}}, \mathrm{d}, J=7.6 \mathrm{~Hz}, 3 \mathrm{H}\right)$, $7.36\left(\mathrm{H}_{\mathrm{B}}, \mathrm{dd}, J=7.6,1.4 \mathrm{~Hz}, 3 \mathrm{H}\right), 5.45\left(\mathrm{H}_{\mathrm{D}}, \mathrm{s}, 1 \mathrm{H}\right), 0.77\left(\mathrm{H}_{\mathrm{E}}, \mathrm{s}, 9 \mathrm{H}\right) ;{ }^{13} \mathrm{C} \mathrm{NMR}\left(100 \mathrm{MHz}, \mathrm{CDCl}_{3}\right)$ $\delta 149.9$ (C5), 147.0 (C6), 133.9 (C3), 133.5 (C1), 129.7 (C10, q, $J=308.5$ Hz), 125.4 (C4), 121.0 (C2), 54.3 (C8), 47.5 (C7), 1.2 (C9); ${ }^{19} \mathrm{~F}$ NMR (376 MHz, $\mathrm{CDCl}_{3}$ ) $\delta$-42.9; HRMS (APCI) $m / z$ : $[\mathrm{M}+\mathrm{H}]^{+}$Calcd for $\mathrm{C}_{26} \mathrm{H}_{20} \mathrm{~F}_{9} \mathrm{~S} 3 \mathrm{Si}$ 627.0347; Found 627.0319.

\section{2-12. Nitration of 1e (Scheme 4)}

To an oven-dried Schlenk tube were added Trip-TMS (1e) $(32.7 \mathrm{mg}, 0.1 \mathrm{mmol})$ and $\mathrm{N}$ nitrosaccharin ( $82.1 \mathrm{mg}, 0.36 \mathrm{mmol})$. TfOH (22.5 mg, $0.15 \mathrm{mmol})$ in DCE (2.0 mL) was added to the tube via syringe, and the mixture was stirred at room temperature for $15 \mathrm{~h}$. The resulting solution was poured into aqueous $\mathrm{NaHCO}_{3}$ solution and extracted with $\mathrm{CHCl}_{3}$ three times. The combined organic layers was dried over $\mathrm{Na}_{2} \mathrm{SO}_{4}$, concentrated in vacuo, and purified by silica gel chromatography (hexane/EtOAc $=20 / 1)$ to give 11 as white solid $(5.1 \mathrm{mg}, 11 \%$ yield).

2,7,14-trinitro-9-(trimethylsilyl)triptycene (11)
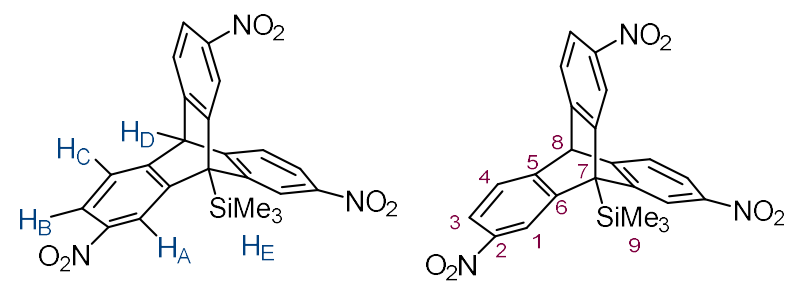

m.p. $>300{ }^{\circ} \mathrm{C} ;{ }^{1} \mathrm{H}$ NMR $\left(400 \mathrm{MHz}, \mathrm{CDCl}_{3}\right) \delta 8.41\left(\mathrm{H}_{\mathrm{A}}, \mathrm{d}, J=1.9 \mathrm{~Hz}, 3 \mathrm{H}\right), 8.04\left(\mathrm{H}_{\mathrm{B}}, \mathrm{dd}, J=8.1\right.$, $1.9 \mathrm{~Hz}, 3 \mathrm{H}), 7.62\left(\mathrm{H}_{\mathrm{C}}, \mathrm{d}, J=8.1 \mathrm{~Hz}, 3 \mathrm{H}\right), 5.72\left(\mathrm{H}_{\mathrm{D}}, \mathrm{s}, 1 \mathrm{H}\right), 0.92\left(\mathrm{H}_{\mathrm{E}}, \mathrm{s}, 9 \mathrm{H}\right) ;{ }^{13} \mathrm{C} \mathrm{NMR}(100 \mathrm{MHz}$, $\mathrm{CDCl}_{3}$ ) $\delta 152.3$ (C5), 146.6 (C2), 145.9 (C6), 125.3 (C4), 122.1 (C3), 120.9 (C1), 54.5 (C8), 47.8 (C7), 1.1 (C9); HRMS (APCI) m/z: [M+H] $]^{+}$Calcd for $\mathrm{C}_{23} \mathrm{H}_{20} \mathrm{~N}_{3} \mathrm{O}_{6} \mathrm{Si} 426.1116$; Found 426.1141. 


\section{Additional Data}

\section{3-1. Trifluoromethylthiolation of 1e (Scheme 4)}

The yield was estimated by ${ }^{1} \mathrm{H}$ NMR and GC-MS analyses. Analytically pure samples of other isomers $(\mathbf{B}, \mathbf{C}, \mathbf{D}$, and $\mathbf{G})$ were isolated by GPC from the combined crude material, and their connectivity was confirmed by HSQC and HMBC analyses.

Table S1. Additional data for the trifluoromethylthiolation of $\mathbf{1 e}$
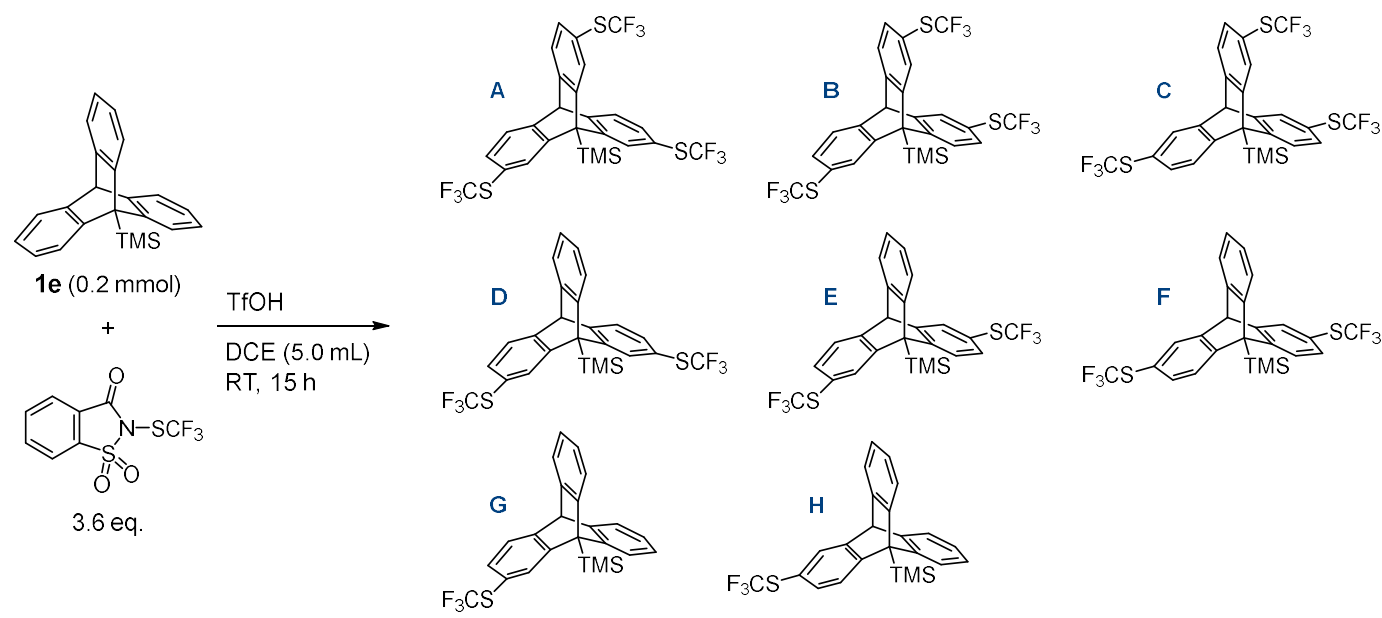

\begin{tabular}{|c|c|c|c|c|c|c|c|c|c|c|}
\hline \multirow[b]{2}{*}{ entry } & \multirow[b]{2}{*}{$\mathrm{TfOH}$ (eq.) } & \multicolumn{9}{|c|}{ NMR yield } \\
\hline & & A & B & C & $\mathrm{D}$ & $E$ & $F$ & G & H & $1 e$ \\
\hline 1 & 1.0 & n.d. & n.d. & n.d. & n.d. & n.d. & n.d. & n.d. & n.d. & $>95 \%$ \\
\hline $2^{a}$ & 1.0 & n.d. & n.d. & n.d. & $11 \%$ & $9 \%$ & $3 \%$ & $67 \%$ & $11 \%$ & $4 \%$ \\
\hline 3 & 3.0 & n.d. & n.d. & n.d. & $27 \%$ & $22 \%$ & $5 \%$ & $37 \%$ & $13 \%$ & n.d. \\
\hline 4 & 6.0 & $3 \%$ & $3 \%$ & trace & $37 \%$ & $27 \%$ & $7 \%$ & $22 \%$ & $8 \%$ & n.d. \\
\hline 5 & 9.0 & $21 \%$ & $26 \%$ & $10 \%$ & $29 \%$ & $20 \%$ & $4 \%$ & n.d. & n.d. & n.d. \\
\hline 6 & 9.0 & $12 \%$ & $14 \%$ & $6 \%$ & $41 \%$ & $30 \%$ & $6 \%$ & n.d. & n.d. & n.d. \\
\hline $6-2^{b}$ & 9.0 & $37 \%$ & $41 \%$ & $15 \%$ & $4 \%$ & $2 \%$ & n.d. & n.d. & n.d. & n.d. \\
\hline
\end{tabular}

[a] With Trip-SMe (1.0 mol\%). [b] The crude mixture of entry 6 was again subjected to the same reaction conditions.

2,6,14-tris(trifluoromethylthio)-9-(trimethylsilyl)triptycene (isomer B)
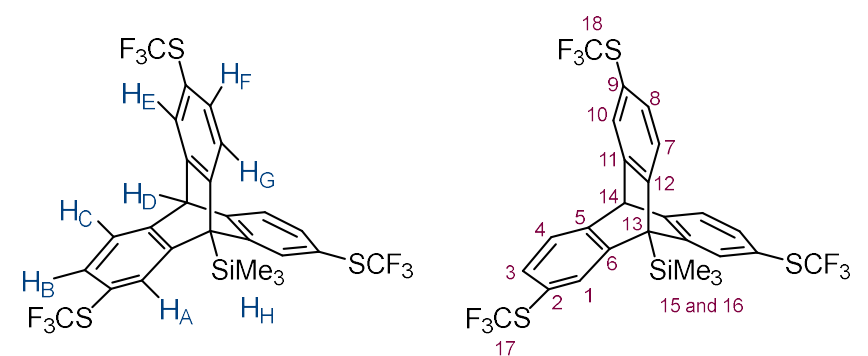

white solid; m.p. 76.6-78.6 ${ }^{\circ} \mathrm{C} ;{ }^{1} \mathrm{H}$ NMR $\left(400 \mathrm{MHz}, \mathrm{CDCl}_{3}\right) \delta 7.76\left(\mathrm{H}_{\mathrm{A}}, \mathrm{s}, 2 \mathrm{H}\right), 7.68\left(\mathrm{H}_{\mathrm{E}}, \mathrm{d}, J=\right.$ $1.7 \mathrm{~Hz}, 1 \mathrm{H}), 7.51\left(\mathrm{H}_{\mathrm{G}}, \mathrm{d}, J=7.9 \mathrm{~Hz}, 1 \mathrm{H}\right), 7.47\left(\mathrm{H}_{\mathrm{C}}, \mathrm{d}, J=7.6 \mathrm{~Hz}, 2 \mathrm{H}\right), 7.37\left(\mathrm{H}_{\mathrm{B}}, \mathrm{dd}, J=5.9,1.5\right.$ 
$\mathrm{Hz}, 2 \mathrm{H}), 7.35\left(\mathrm{H}_{\mathrm{F}}, \mathrm{dd}, J=6.2,1.9 \mathrm{~Hz}, 1 \mathrm{H}\right), 5.44\left(\mathrm{H}_{\mathrm{D}}, \mathrm{s}, 1 \mathrm{H}\right), 0.78\left(\mathrm{H}_{\mathrm{H}}, \mathrm{s}, 9 \mathrm{H}\right) ;{ }^{13} \mathrm{C}$ NMR $(100$ $\mathrm{MHz}, \mathrm{CDCl}_{3}$ ) $\delta 150.0(\mathrm{C} 5), 148.6(\mathrm{C} 11), 148.5$ (C12), 146.9 (C6), 134.0 (C3), 133.6 (C8), 133.5 (C1), 131.8 (C10), 129.7 (C17 or C18, q, $J=308.4 \mathrm{~Hz}$ ), 129.7 (C17 or C18, q, $J=307.7 \mathrm{~Hz}$ ), 126.5 (C7), 125.4 (C4), 121.2 (C9), 120.9 (C2), 54.2 (C14), 47.6 (C13), 1.4 (C15), 1.2 (C16); ${ }^{19} \mathrm{~F}$ NMR (376 MHz, $\mathrm{CDCl}_{3}$ ) $\delta$-42.5, -42.9; HRMS (EI) $\mathrm{m} / z$ : $[\mathrm{M}]^{+}$Calcd for $\mathrm{C}_{26} \mathrm{H}_{19} \mathrm{~F}_{9} \mathrm{~S}_{3} \mathrm{Si}$ 626.0279; Found 626.0263.

3,6,14-tris(trifluoromethylthio)-9-(trimethylsilyl)triptycene (isomer C)
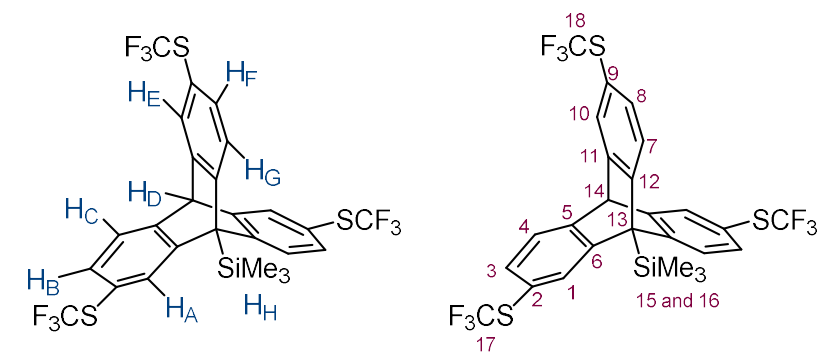

white solid; m.p. 183.2-185.2 ${ }^{\circ} \mathrm{C} ;{ }^{1} \mathrm{H}$ NMR $\left(400 \mathrm{MHz}, \mathrm{CDCl}_{3}\right) \delta 7.75\left(\mathrm{H}_{\mathrm{A}}, \mathrm{s}, 1 \mathrm{H}\right), 7.68\left(\mathrm{H}_{\mathrm{E}}, \mathrm{d}, J=\right.$ $1.8 \mathrm{~Hz}, 2 \mathrm{H}), 7.50\left(\mathrm{H}_{\mathrm{G}}, \mathrm{d}, J=8.0 \mathrm{~Hz}, 2 \mathrm{H}\right), 7.48\left(\mathrm{H}_{\mathrm{C}}, \mathrm{d}, J=7.6 \mathrm{~Hz}, 1 \mathrm{H}\right), 7.37\left(\mathrm{H}_{\mathrm{B}}, \mathrm{dd}, J=7.9,1.5\right.$ $\mathrm{Hz}, 1 \mathrm{H}), 7.43\left(\mathrm{H}_{\mathrm{F}}, \mathrm{dd}, J=8.0,1.8 \mathrm{~Hz}, 2 \mathrm{H}\right), 5.42\left(\mathrm{H}_{\mathrm{D}}, \mathrm{s}, 1 \mathrm{H}\right), 0.77\left(\mathrm{H}_{\mathrm{H}}, \mathrm{s}, 9 \mathrm{H}\right) ;{ }^{13} \mathrm{C} \mathrm{NMR}(100$ $\mathrm{MHz}, \mathrm{CDCl}_{3}$ ) $\delta 150.1$ (C5), 148.6 (C12), 148.5 (C11), 146.8 (C6), 134.0 (C3), 133.6 (C8), 133.5, (C1) 131.8 (C10), 129.7 (C17 or C18, q, $J=308.0 \mathrm{~Hz}$ ), 129.6 (C17 or C18, q, $J=308.3 \mathrm{~Hz}), 126.5$ (C7), 125.4 (C4), 121.3 (C9), 120.8 (C2), 54.0 (C14), 47.6 (C13), 1.7 (C15), 1.5 (C16); ${ }^{19} \mathrm{~F}$ NMR $\left(376 \mathrm{MHz}, \mathrm{CDCl}_{3}\right) \delta-42.5,-42.9$; HRMS (APCI) $m / z$ : $[\mathrm{M}+\mathrm{H}]^{+}$Calcd for $\mathrm{C}_{26} \mathrm{H}_{20} \mathrm{~F}_{9} \mathrm{~S}_{3} \mathrm{Si}$ 627.0347; Found 627.0320 .

2,7-bis(trifluoromethylthio)-9-(trimethylsilyl)triptycene (isomer D)
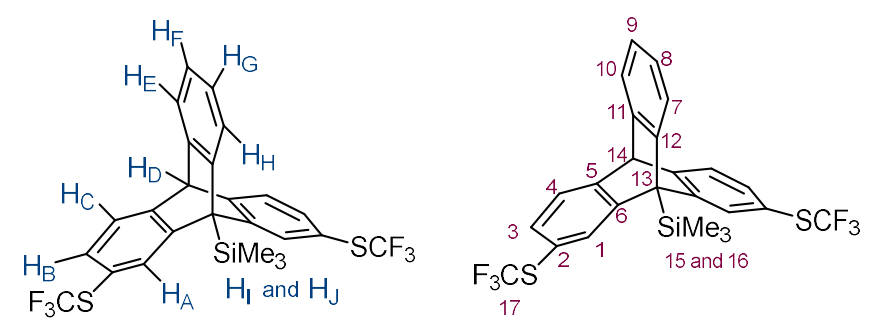

white solid; m.p. 178.6-180.6 ${ }^{\circ} \mathrm{C} ;{ }^{1} \mathrm{H}$ NMR $\left(400 \mathrm{MHz}, \mathrm{CDCl}_{3}\right) \delta 7.75\left(\mathrm{H}_{\mathrm{A}}, \mathrm{s}, 2 \mathrm{H}\right), 7.50-7.48\left(\mathrm{H}_{\mathrm{H}}\right.$, $\mathrm{m}, 1 \mathrm{H}), 7.45-7.41\left(\mathrm{H}_{\mathrm{C}}\right.$ and $\left.\mathrm{H}_{\mathrm{E}}, \mathrm{m}, 3 \mathrm{H}\right), 7.33\left(\mathrm{H}_{\mathrm{B}}, \mathrm{dd}, J=7.6,1.5 \mathrm{~Hz}, 2 \mathrm{H}\right), 7.06-7.04\left(\mathrm{H}_{\mathrm{F}}\right.$ and $\mathrm{H}_{\mathrm{G}}$, m, 2H), $5.41\left(\mathrm{H}_{\mathrm{D}}, \mathrm{s}, 1 \mathrm{H}\right), 0.78\left(\mathrm{H}_{\mathrm{I}}, \mathrm{s}, 6 \mathrm{H}\right), 0.77\left(\mathrm{H}_{\mathrm{J}}, \mathrm{s}, 3 \mathrm{H}\right) ;{ }^{13} \mathrm{C} \mathrm{NMR}\left(100 \mathrm{MHz}, \mathrm{CDCl}_{3}\right) \delta 150.8$ (C5), 147.6 (C6), 146.8 (C11), 145.3 (C12), 133.7 (C3), 133.3 (C1), 129.7 (C17, q, J=307.7 Hz), 
125.7 (C7), 125.5 (C8), 125.4 (C9), 125.2 (C4), 124.6 (C10), 120.4 (C2), 54.7 (C14), 47.6 (C13), 1.5 (C15), $1.3(\mathrm{C} 16) ;{ }^{19} \mathrm{~F}$ NMR (376 MHz, $\mathrm{CDCl}_{3}$ ) $\delta$-43.0; HRMS (APCI) $\mathrm{m} / \mathrm{z}$ : $[\mathrm{M}+\mathrm{H}]^{+} \mathrm{Calcd}$ for $\mathrm{C}_{25} \mathrm{H}_{21} \mathrm{~F}_{6} \mathrm{~S}_{2} \mathrm{Si}$ 527.0753; Found 527.0770.

2-(trifluoromethylthio)-9-(trimethylsilyl)triptycene (isomer G)
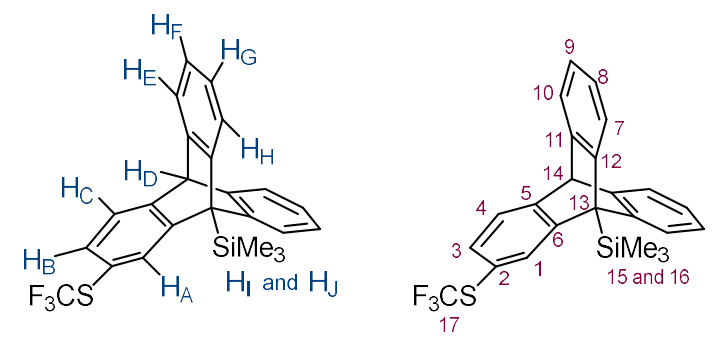

white solid; m.p. 251.2-253.2 ${ }^{\circ} \mathrm{C} ;{ }^{1} \mathrm{H}$ NMR $\left(400 \mathrm{MHz}, \mathrm{CDCl}_{3}\right) \delta 7.73\left(\mathrm{H}_{\mathrm{A}}, \mathrm{s}, 1 \mathrm{H}\right), 7.48-7.46\left(\mathrm{H}_{\mathrm{H}}\right.$, $\mathrm{m}, 2 \mathrm{H}), 7.42-7.39\left(\mathrm{H}_{\mathrm{E}}\right.$ and $\left.\mathrm{H}_{\mathrm{C}}, \mathrm{m}, 3 \mathrm{H}\right), 7.29\left(\mathrm{H}_{\mathrm{B}}, \mathrm{dd}, J=7.6,1.6 \mathrm{~Hz}, 1 \mathrm{H}\right), 7.04-6.98\left(\mathrm{H}_{\mathrm{F}}\right.$ and $\mathrm{H}_{\mathrm{G}}$, m, 4H), $5.37\left(\mathrm{H}_{\mathrm{D}}, \mathrm{s}, 1 \mathrm{H}\right), 0.77\left(\mathrm{H}_{\mathrm{I}}, \mathrm{s}, 3 \mathrm{H}\right), 0.76\left(\mathrm{H}_{\mathrm{J}}, \mathrm{s}, 6 \mathrm{H}\right) ;{ }^{13} \mathrm{C} \mathrm{NMR}\left(100 \mathrm{MHz}, \mathrm{CDCl}_{3}\right) \delta 151.7$ (C5), 148.3 (C6), 147.6 (C11), 145.9 (C12), 133.4 (C3), 133.1 (C1), 129.8 (C17, q, J=308.1 Hz), 125.5 (C7), 125.2 (C9), 124.9 (C4 and C8), 124.4 (C10), 119.7 (C2), 55.0 (C14), 47.7 (C13), 1.8 (C15), $1.6(\mathrm{C} 16) ;{ }^{19} \mathrm{~F}$ NMR (376 MHz, $\left.\mathrm{CDCl}_{3}\right) \delta-43.0$; HRMS (APCI) $m / z$ : $[\mathrm{M}+\mathrm{H}]^{+} \mathrm{Calcd}$ for $\mathrm{C}_{24} \mathrm{H}_{22} \mathrm{~F}_{3} \mathrm{SSi}$ 427.1158; Found 427.1145. 


\section{3-2. Nitration of 1e (Scheme 4)}

The yield was estimated by ${ }^{1} \mathrm{H}$ NMR and GC-MS analyses. Analytically pure samples of other isomers $(\mathbf{J}, \mathbf{K}, \mathbf{O}$, and $\mathbf{P}$ ) were isolated by GPC from the combined crude material, and their connectivity was confirmed by HSQC and HMBC analyses.

Table S2. Additional data for the nitration of 1e
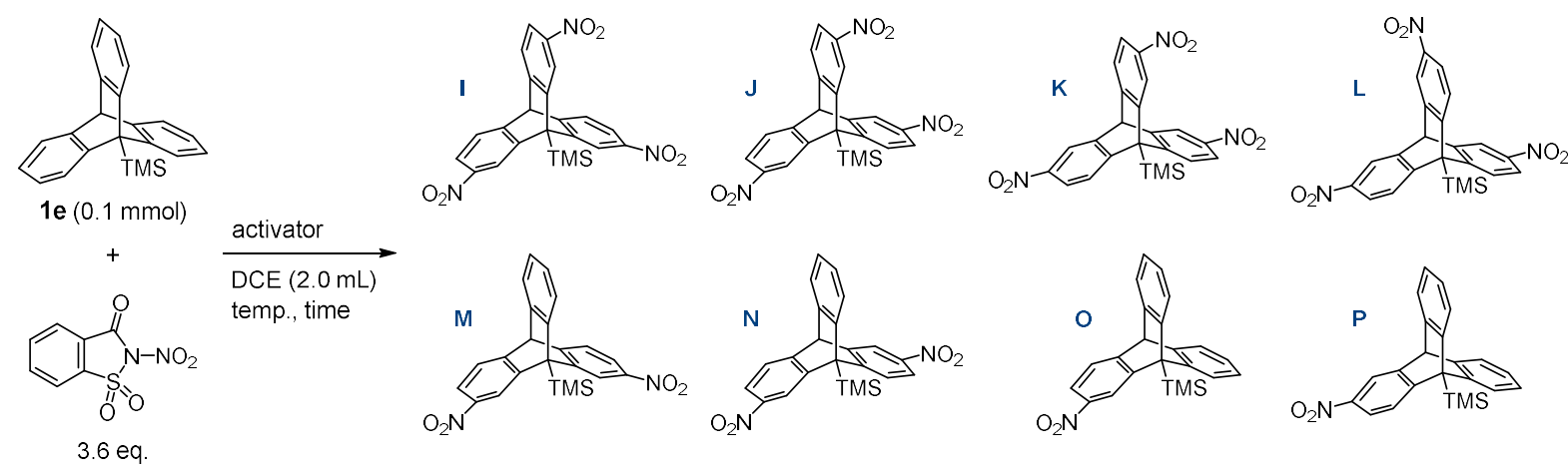

\begin{tabular}{|c|c|c|c|c|c|c|c|c|c|c|c|}
\hline \multirow[b]{2}{*}{ entry } & \multirow[b]{2}{*}{ activator (eq.) } & \multirow[b]{2}{*}{ temp. } & \multirow[b]{2}{*}{ time (h) } & \multicolumn{8}{|c|}{ NMR yield (\%) } \\
\hline & & & & I & J & $\mathrm{K}$ & L & $M+N$ & 0 & $\mathbf{P}$ & $1 e$ \\
\hline 1 & $\mathrm{TfOH}(1.5)$ & $\mathrm{RT}$ & 4 & $15 \%$ & $37 \%$ & $33 \%$ & $11 \%$ & n.d. & n.d. & n.d. & n.d. \\
\hline 2 & $\mathrm{TfOH}(1.5)$ & $0^{\circ} \mathrm{C}$ & 15 & $16 \%$ & $38 \%$ & $33 \%$ & $10 \%$ & n.d. & n.d. & n.d. & n.d. \\
\hline 3 & $\mathrm{TfOH}(1.5)$ & $-20^{\circ} \mathrm{C}$ & 15 & $18 \%$ & $37 \%$ & $32 \%$ & $10 \%$ & n.d. & n.d. & n.d. & n.d. \\
\hline 4 & $\mathrm{TfOH}(1.5)$ & $\mathrm{RT}$ & 15 & $18 \%$ & $38 \%$ & $34 \%$ & $8 \%$ & n.d. & n.d. & n.d. & n.d. \\
\hline 5 & $\mathrm{MsOH}(1.5)$ & RT & 15 & n.d. & n.d. & n.d. & n.d. & $13 \%$ & $52 \%$ & $39 \%$ & trace \\
\hline 7 & $\mathrm{MsOH}(3.0)$ & $\mathrm{RT}$ & 15 & $21 \%$ & $45 \%$ & $37 \%$ & $10 \%$ & n.d. & n.d. & n.d. & n.d. \\
\hline 8 & TFA (1.5) & RT & 15 & n.d. & n.d. & n.d. & n.d. & n.d. & trace & trace & $>95 \%$ \\
\hline
\end{tabular}

2,6,14-trinitro-9-(trimethylsilyl)triptycene (isomer $\mathbf{J}$ )
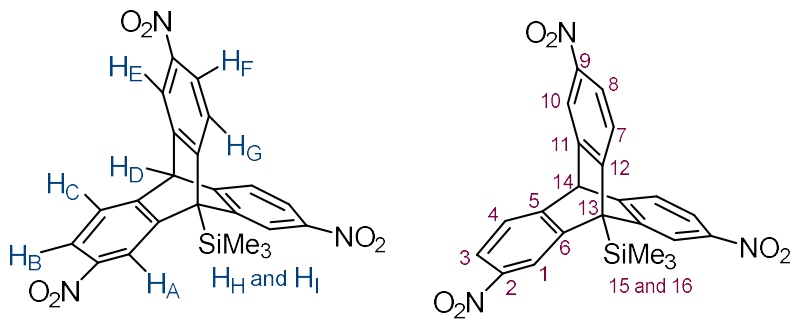

white solid; m.p. 171.4-173.4 ${ }^{\circ} \mathrm{C}$; ${ }^{1} \mathrm{H}$ NMR $\left(400 \mathrm{MHz}, \mathrm{CDCl}_{3}\right) \delta 8.40\left(\mathrm{H}_{\mathrm{A}}, \mathrm{d}, J=2.1 \mathrm{~Hz}, 2 \mathrm{H}\right), 8.31$ $\left(\mathrm{H}_{\mathrm{E}}, \mathrm{d}, J=2.3 \mathrm{~Hz}, 1 \mathrm{H}\right), 8.04\left(\mathrm{H}_{\mathrm{B}}, \mathrm{dd}, J=8.0,2.1 \mathrm{~Hz}, 2 \mathrm{H}\right), 8.02\left(\mathrm{H}_{\mathrm{F}}, \mathrm{dd}, J=8.3,2.3 \mathrm{~Hz}, 1 \mathrm{H}\right), 7.67$ $\left(\mathrm{H}_{\mathrm{G}}, \mathrm{d}, J=8.4 \mathrm{~Hz}, 1 \mathrm{H}\right), 7.64\left(\mathrm{H}_{\mathrm{C}}, \mathrm{d}, J=8.0 \mathrm{~Hz}, 2 \mathrm{H}\right), 5.74\left(\mathrm{H}_{\mathrm{D}}, \mathrm{s}, 1 \mathrm{H}\right), 0.90\left(\mathrm{H}_{\mathrm{H}}, \mathrm{s}, 3 \mathrm{H}\right), 0.88\left(\mathrm{H}_{\mathrm{I}}\right.$, s, 6H); ${ }^{13} \mathrm{C}$ NMR (100 MHz, $\mathrm{CDCl}_{3}$ ) $\delta 152.7$ (C5), 152.0 (C12), 147.4 (C11), 146.2 (C2), 145.9 (C6), 145.6 (C9), 126.3 (C7), 125.3 (C4), 122.1 (C3), 121.8 (C8), 121.0 (C1), 119.8 (C10), 54.4 
(C14), 48.1 (C13), 1.4 (C15), 1.2 (C16); HRMS (APCI) $m / z$ : $[\mathrm{M}+\mathrm{H}]^{+}$Calcd for $\mathrm{C}_{23} \mathrm{H}_{20} \mathrm{~N}_{3} \mathrm{O}_{6} \mathrm{Si}$ 426.1116; Found 426.1140.

3,6,14-trinitro-9-(trimethylsilyl)triptycene (isomer $\mathbf{K}$ )
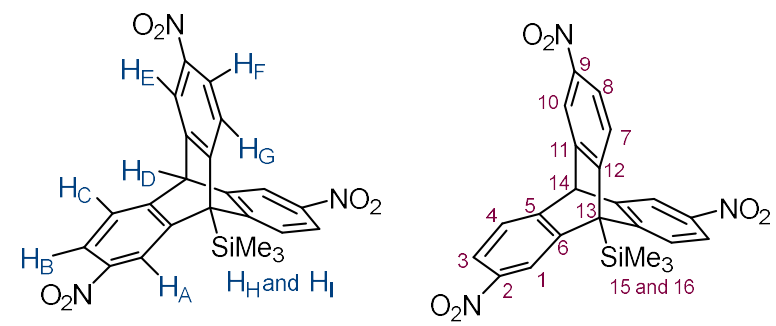

white solid; m.p. $172.3-174.3{ }^{\circ} \mathrm{C} ;{ }^{1} \mathrm{H}$ NMR $\left(400 \mathrm{MHz}, \mathrm{CDCl}_{3}\right) \delta 8.39\left(\mathrm{H}_{\mathrm{A}}, \mathrm{d}, J=2.0 \mathrm{~Hz}, 1 \mathrm{H}\right), 8.32$ $\left(\mathrm{H}_{\mathrm{E}}, \mathrm{d}, J=2.3 \mathrm{~Hz}, 2 \mathrm{H}\right), 8.05\left(\mathrm{H}_{\mathrm{B}}, \mathrm{dd}, J=8.2,2.1 \mathrm{~Hz}, 1 \mathrm{H}\right), 8.01 \mathrm{H}_{\mathrm{F}},(\mathrm{dd}, J=8.4,2.3 \mathrm{~Hz}, 2 \mathrm{H}), 7.66$ $\left(\mathrm{H}_{\mathrm{G}}, \mathrm{d}, J=8.4 \mathrm{~Hz}, 2 \mathrm{H}\right), 7.65\left(\mathrm{H}_{\mathrm{C}}, \mathrm{d}, J=8.1 \mathrm{~Hz}, 1 \mathrm{H}\right), 5.75\left(\mathrm{H}_{\mathrm{D}}, \mathrm{s}, 1 \mathrm{H}\right), 0.87\left(\mathrm{H}_{\mathrm{H}}, \mathrm{s}, 6 \mathrm{H}\right), 0.85\left(\mathrm{H}_{\mathrm{I}}\right.$, s, 3H); ${ }^{13} \mathrm{C}$ NMR (100 MHz, $\left.\mathrm{CDCl}_{3}\right) \delta 153.1$ (C5), 151.5 (C12), 147.7 (C6), 145.9 (C2), 145.8 (C9), 145.6 (C11), 126.4 (C7), 125.3 (C4), 122.2 (C3), 121.7 (C8), 121.0 (C1), 119.7 (C10), 54.2 (C14), 48.4 (C13), 1.6 (C15), 1.4 (C16); HRMS (APCI) $m / z:[\mathrm{M}+\mathrm{H}]^{+}$Calcd for $\mathrm{C}_{23} \mathrm{H}_{20} \mathrm{~N}_{3} \mathrm{O}_{6} \mathrm{Si}$ 426.1116; Found 426.1142.

2-nitro-9-(trimethylsilyl)triptycene (isomer $\mathbf{O}$ )
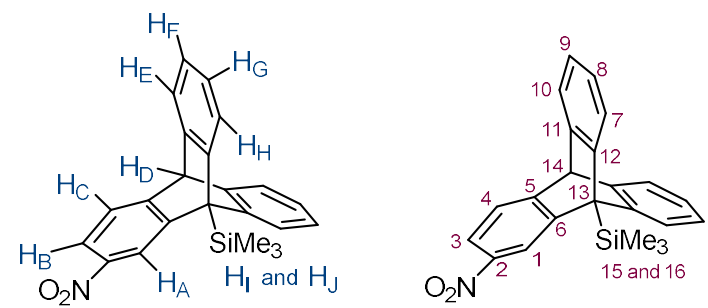

white solid; m.p. 207.8-209.8 ${ }^{\circ} \mathrm{C}$; ${ }^{1} \mathrm{H}$ NMR $\left(400 \mathrm{MHz}, \mathrm{CDCl}_{3}\right) \delta 8.33\left(\mathrm{H}_{\mathrm{A}}, \mathrm{d}, J=2.1 \mathrm{~Hz}, 1 \mathrm{H}\right), 7.93$ $\left(\mathrm{H}_{\mathrm{B}}, \mathrm{dd}, J=8.1,2.1 \mathrm{~Hz}, 1 \mathrm{H}\right), 7.51-7.48\left(\mathrm{H}_{\mathrm{C}}\right.$ and $\left.\mathrm{H}_{\mathrm{H}}, \mathrm{m}, 3 \mathrm{H}\right), 7.43-7.41\left(\mathrm{H}_{\mathrm{E}}, \mathrm{m}, 2 \mathrm{H}\right), 7.06-7.00\left(\mathrm{H}_{\mathrm{F}}\right.$ and $\left.\mathrm{H}_{\mathrm{G}}, \mathrm{m}, 4 \mathrm{H}\right), 5.45\left(\mathrm{H}_{\mathrm{D}}, \mathrm{s}, 1 \mathrm{H}\right), 0.81\left(\mathrm{H}_{\mathrm{I}}, \mathrm{s}, 6 \mathrm{H}\right), 0.80\left(\mathrm{H}_{\mathrm{J}}, \mathrm{s}, 3 \mathrm{H}\right) ;{ }^{13} \mathrm{C} \mathrm{NMR}\left(100 \mathrm{MHz}, \mathrm{CDCl}_{3}\right)$ ઈ 155.9 (C5), 148.5 (C2), 146.8 (C11), 145.5 (C12), 145.1 (C6), 125.6 (C9), 125.5 (C7), 125.2 (C8), 124.6 (C10), 124.2 (C4), 121.1 (C3), 120.2 (C1), 55.1 (C14), 47.8 (C13), 1.7 (C15), 1.7 (C16); HRMS (APCI) $m / z$ : [M+H] $]^{+}$Calcd for $\mathrm{C}_{23} \mathrm{H}_{22} \mathrm{NO}_{2} \mathrm{Si} 372.1414$; Found 372.1429.

3-nitro-9-(trimethylsilyl)triptycene (isomer P) 

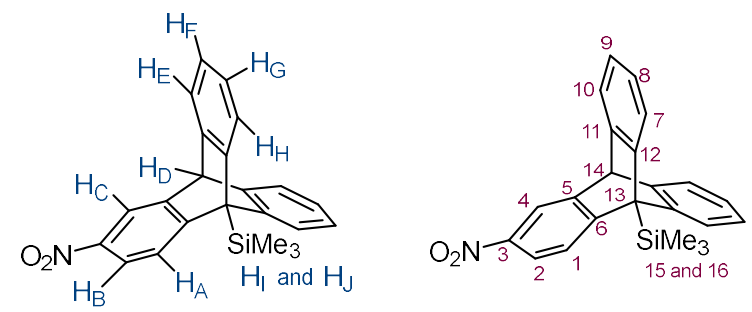

white solid; m.p. 285.6-297.6 ${ }^{\circ} \mathrm{C} ;{ }^{1} \mathrm{H}$ NMR $\left(400 \mathrm{MHz}, \mathrm{CDCl}_{3}\right) \delta 8.21\left(\mathrm{H}_{\mathrm{C}}, \mathrm{d}, J=2.3 \mathrm{~Hz}, 1 \mathrm{H}\right), 7.90$ $\left(\mathrm{H}_{\mathrm{B}}, \mathrm{dd}, J=8.3,2.4 \mathrm{~Hz}, 1 \mathrm{H}\right), 7.56\left(\mathrm{H}_{\mathrm{A}}, \mathrm{d}, J=8.5 \mathrm{~Hz}, 1 \mathrm{H}\right), 7.49-7.47\left(\mathrm{H}_{\mathrm{H}}, \mathrm{m}, 2 \mathrm{H}\right), 7.45-7.43\left(\mathrm{H}_{\mathrm{E}}\right.$, m, 2H), 7.06-6.99 ( $\mathrm{H}_{\mathrm{F}}$ and $\left.\mathrm{H}_{\mathrm{G}}, \mathrm{m}, 4 \mathrm{H}\right), 5.46\left(\mathrm{H}_{\mathrm{D}}, \mathrm{s}, 1 \mathrm{H}\right), 0.78\left(\mathrm{H}_{\mathrm{I}}, \mathrm{s}, 3 \mathrm{H}\right), 0.77\left(\mathrm{H}_{\mathrm{J}}, \mathrm{s}, 6 \mathrm{H}\right) ;{ }^{13} \mathrm{C}$ NMR (100 MHz, $\mathrm{CDCl}_{3}$ ) $\delta 154.4$ (C6), 150.4 (C5), 147.2 (C11), 145.0 (C12), 144.9 (C3), 125.7 (C9), 125.5 (C7), 125.4 (C1), 125.2 (C8), 124.5 (C10), 120.6 (C4), 118.8 (C2), 54.9 (C14), 48.2 (C13), 1.9 (C15), 1.8 (C16); HRMS (APCI) m/z: [M+H] $]^{+}$Calcd for $\mathrm{C}_{23} \mathrm{H}_{22} \mathrm{NO}_{2} \mathrm{Si}$ 372.1414; Found 372.1443 . 


\section{X-Ray Crystallographic Analysis}

Single crystals of $\mathbf{2 e}$ and $\mathbf{2 f}$ suitable for X-ray analysis were obtained by $n$-pentane vapor diffusion into the $\mathrm{CHCl}_{3}$ solution. The structures were refined by full-matrix least-squares method using SHELXL-2016/6. ${ }^{8}$ Hydrogen atoms were included in the refinement on calculated positions riding on their carrier atoms. ORTEP-3 programs were used to draw the molecules. ${ }^{9}$ The CCDC numbers are 2067724 (2e) and 2067725 (2f). These data can be obtained free of charge from the Cambridge Crystallographic Data Centre via www.ccdc.cam.ac.uk/data_request/cif.

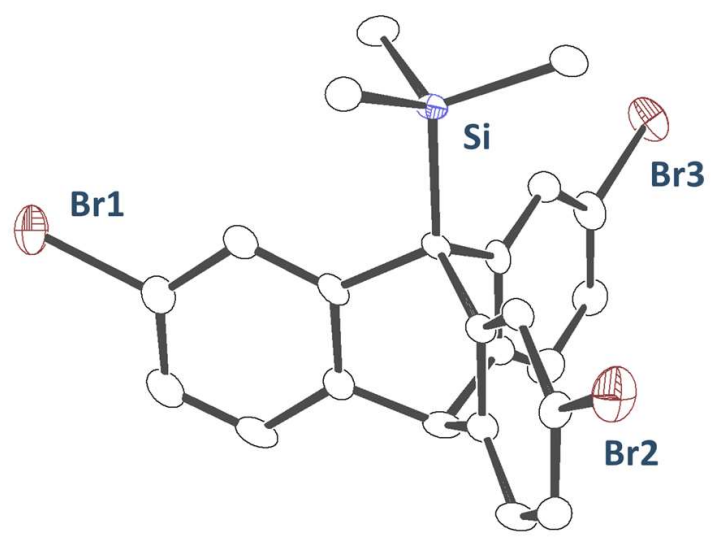

Figure S1. ORTEP drawing for 2e with 50\% thermal ellipsoid. Hydrogen atoms are omitted for clarity.

Table S3. Crystal data for $2 \mathrm{e}$

Crystal system

Space group

Unit cell parameter $[\AA, \operatorname{deg}]$

Cell volume $\left[\AA^{3}\right]$

$\mathrm{Z}$

$\mathrm{R}$ factor $(I>2.0 \sigma(I))$

$\mathrm{R}$ factor (all data)

Rint

Goodness of fit monoclinic

C 2 (No. 5)

$a=23.9934(5)$

$b=9.0853(2), \beta=101.573(2)$

$c=10.0477(2)$

2145.74(8)

4

$R 1=0.0355, w R 2=0.0916$

$R 1=0.0360, w R 2=0.0939$

0.0394

1.078 


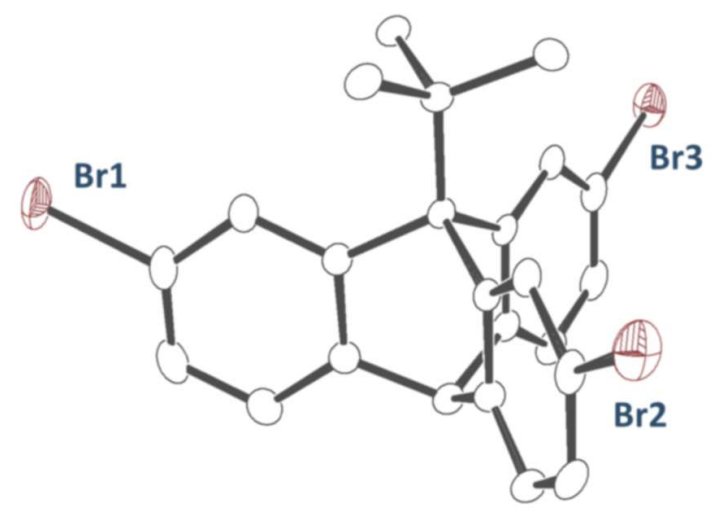

Figure S1. ORTEP drawing for $\mathbf{2 f}$ with $40 \%$ thermal ellipsoid. Hydrogen atoms and solvent molecules are omitted for clarity.

Table S3. Crystal data for $\mathbf{2 f}$

Crystal system

Space group

Unit cell parameter $[\AA$, deg]

Cell volume $\left[\AA^{3}\right]$

$\mathrm{Z}$

$\mathrm{R}$ factor $(I>2.0 \sigma(I))$

$\mathrm{R}$ factor (all data)

Rint

Goodness of fit monoclinic

$P 21 / n$ (No. 14)

$a=10.05210(10)$

$b=8.63450(10), \beta=96.9450(10)$

$c=28.2011(3)$

2429.75(5)

4

$R 1=0.0566, w R 2=0.1584$

$R 1=0.0582, w R 2=0.1607$

0.0941

1.043 


\section{Computational Study}

All calculations were carried out using the Gaussian 16 program, ${ }^{10}$ and geometries of all optimized structures are summarized in an XYZ file. Geometry optimizations and frequency calculations were conducted at the M06-X2/6-311+G(d.p) level of theory, and the solvent effect of DCE was taken account by the integral equation formalism PCM (IEF-PCM). The optimized molecular structures were verified by vibrational analysis to have no imaginary frequency. The Hirshfeld population analysis was conducted at the B3LYP/6-311++G(3df,2pd) level for the optimized geometries.

Table S4. Summary of Hirshfeld charge of triptycene derivatives

\begin{tabular}{|c|c|c|c|c|c|c|c|}
\hline $\mathrm{R}$ & & $q[\mathrm{e}]$ & $\Delta q[\mathrm{e}]$ & $\mathrm{R}$ & & $q[\mathrm{e}]$ & $\Delta q[\mathrm{e}]$ \\
\hline $\mathrm{H}$ & $\mathrm{C} 2$ & -0.042938 & -- & \multirow{2}{*}{ TMS } & $\mathrm{C} 2$ & -0.044136 & -0.001198 \\
\hline \multirow{2}{*}{$\mathrm{Br}$} & $\mathrm{C} 2$ & -0.039380 & +0.003558 & & $\mathrm{C} 3$ & -0.043060 & -0.000122 \\
\hline & C3 & -0.038930 & +0.004008 & \multirow{6}{*}{$\mathrm{tBu}$} & $\mathrm{C} 2$ & -0.045185 & -0.002247 \\
\hline \multirow{4}{*}{ SMe } & $\mathrm{C} 2$ & -0.042670 & +0.000268 & & $C 2^{\prime}$ & -0.044936 & -0.001998 \\
\hline & $C 2^{\prime}$ & -0.040691 & +0.002247 & & $C 2{ }^{\prime \prime}$ & -0.045186 & -0.002248 \\
\hline & C3 & -0.041160 & +0.001778 & & $\mathrm{C} 3$ & -0.042553 & +0.000385 \\
\hline & $\mathrm{C}^{\prime}$ & -0.041029 & +0.001909 & & $C 3^{\prime}$ & -0.042993 & -0.000055 \\
\hline \multirow{4}{*}{ OMe } & $\mathrm{C} 2$ & -0.042709 & +0.000229 & & C3" & -0.042553 & +0.000385 \\
\hline & $C 2^{\prime}$ & -0.041795 & +0.001148 & \multirow{2}{*}{$\mathrm{Me}$} & $\mathrm{C} 2$ & -0.043654 & -0.000716 \\
\hline & C3 & -0.041847 & +0.001091 & & $\mathrm{C} 3$ & -0.043052 & -0.000114 \\
\hline & C3' & -0.043213 & -0.000275 & & & & \\
\hline
\end{tabular}

Figure S3. Relative stability of protonated species of Trip-TMS (1e) and Trip-t-Bu (1f).
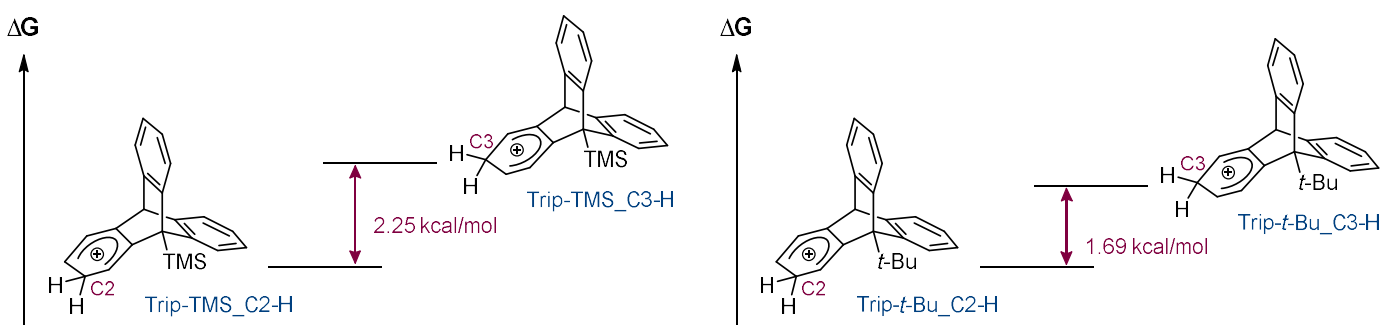
Figure S4. Summary of the change in bond lengths

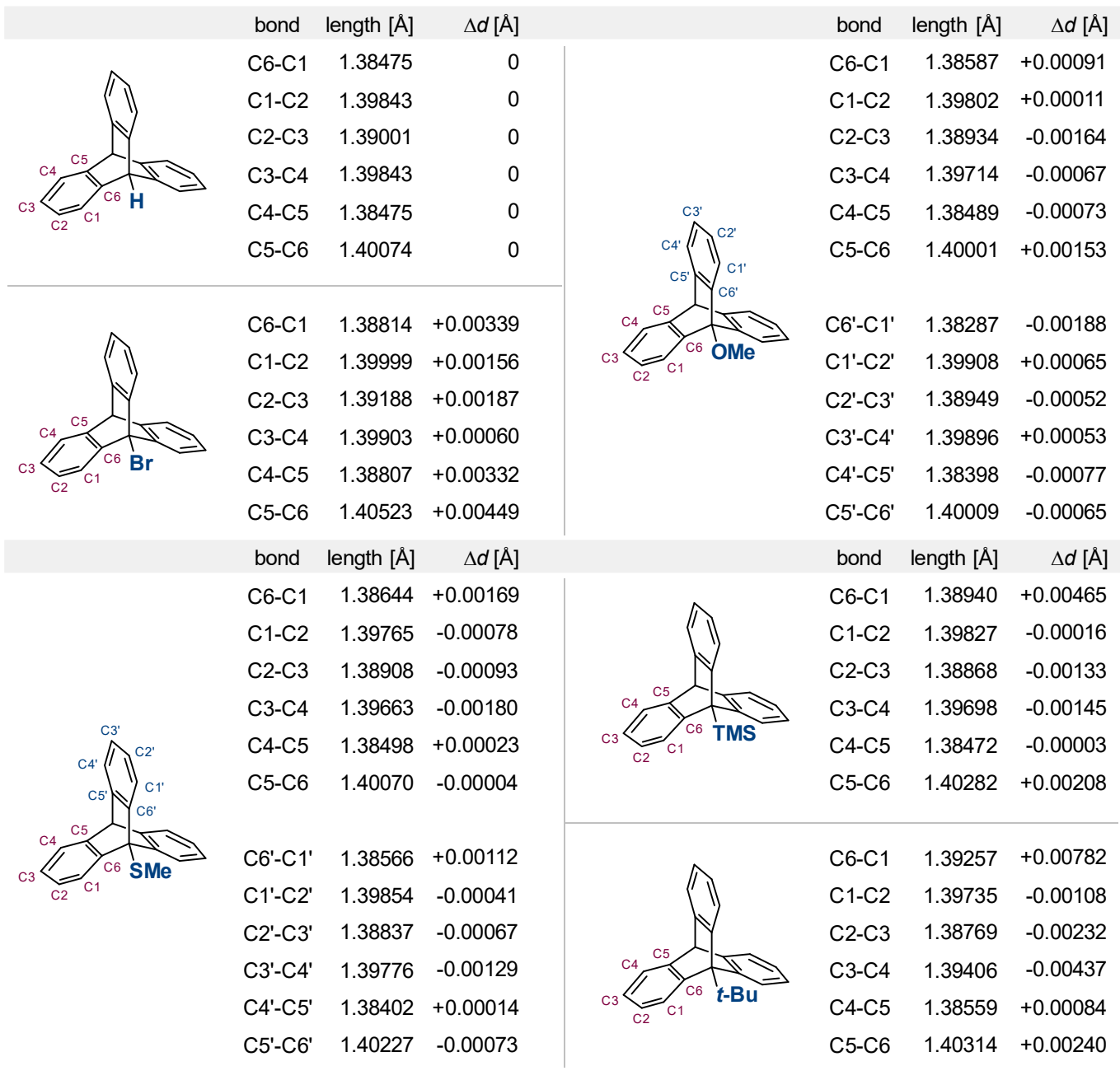

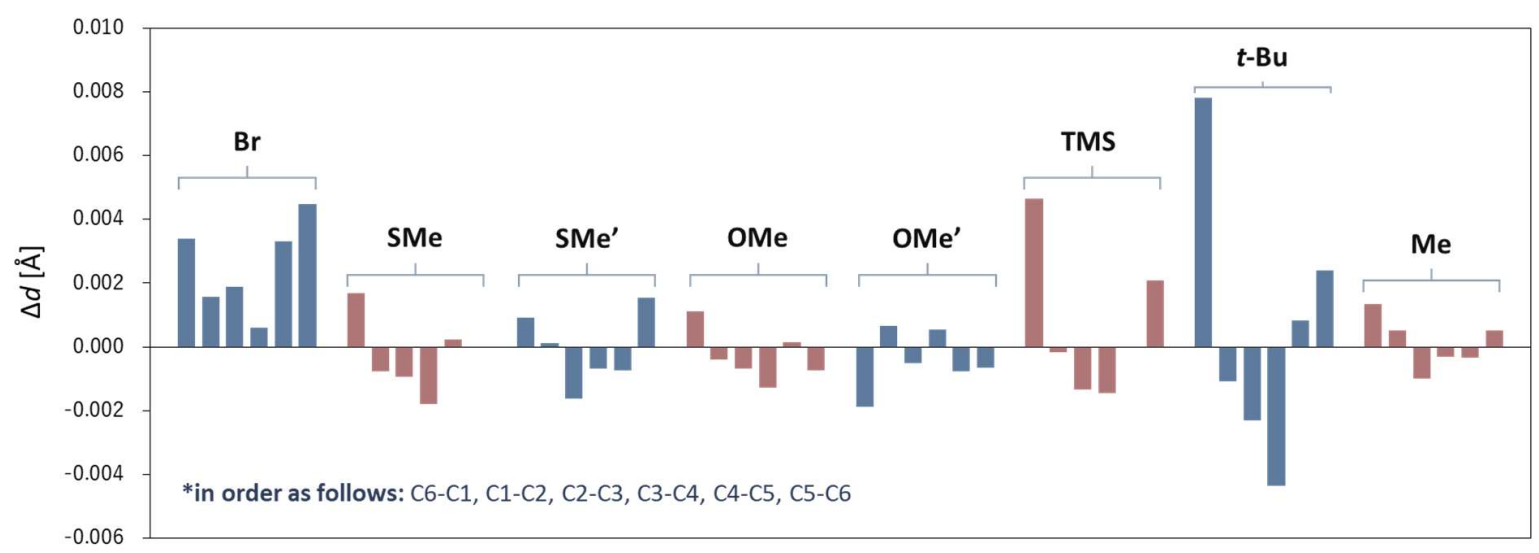


Table S5. Summary of the calculation at the M06-X2/6-311+G(d.p)/PCM(DCE) level.

\begin{tabular}{|l|l|l|}
\hline & $\begin{array}{l}\text { EE }+ \text { Thermal Enthalpy } \\
\text { Correction [Hartree] }\end{array}$ & $\begin{array}{l}\text { EE + Thermal Free Energy } \\
\text { Correction [Hartree] }\end{array}$ \\
\hline Trip-H (1a) & -770.15751 & -770.21047 \\
\hline Trip-SMe (1b) & -1207.6098 & -1207.6700 \\
\hline Trip-OMe (1c) & -884.62782 & -884.68607 \\
\hline Trip-Br (1d) & -3344.0188 & -3344.0765 \\
\hline Trip-TMS (1e) & -1178.6743 & -1178.7441 \\
\hline Trip-t-Bu (1f) & -927.23405 & -927.29775 \\
\hline Trip-Me & -809.4358 & -809.49157 \\
\hline Trip-TMS_C2-H & -1179.0357 & -1179.1088 \\
\hline Trip-TMS_C3-H & -1179.0346 & -1179.1052 \\
\hline Trip-t-Bu_C2-H & -927.59585 & -927.66107 \\
\hline Trip-t-Bu_C3-H & -927.59397 & -927.65846 \\
\hline
\end{tabular}




\section{Copy of NMR Spectra}

${ }^{1} \mathrm{H}$ NMR of Trip-TMS (1e) (400 MHz, $\mathrm{CDCl}_{3}$ )

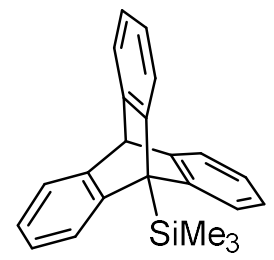

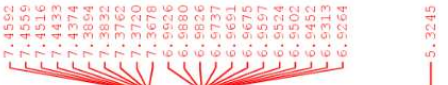

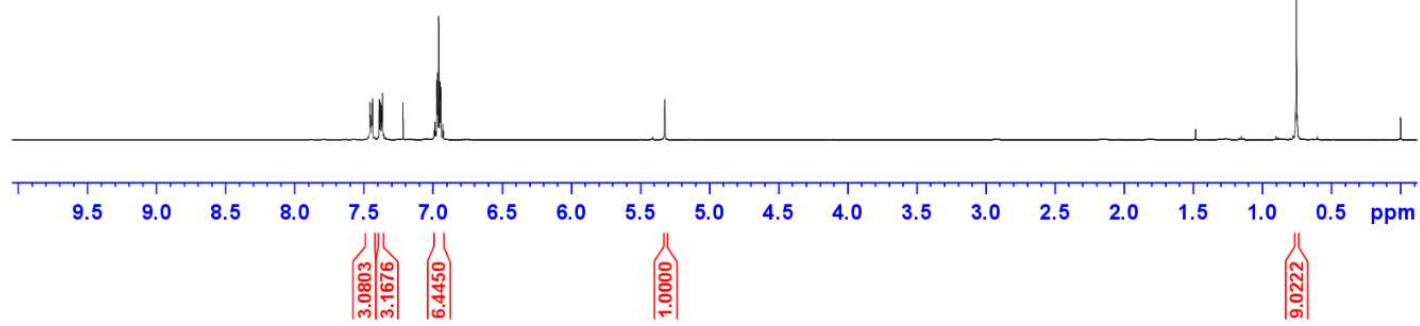

${ }^{13} \mathrm{C}$ NMR of Trip-TMS (1e) $\left(100 \mathrm{MHz}, \mathrm{CDCl}_{3}\right)$
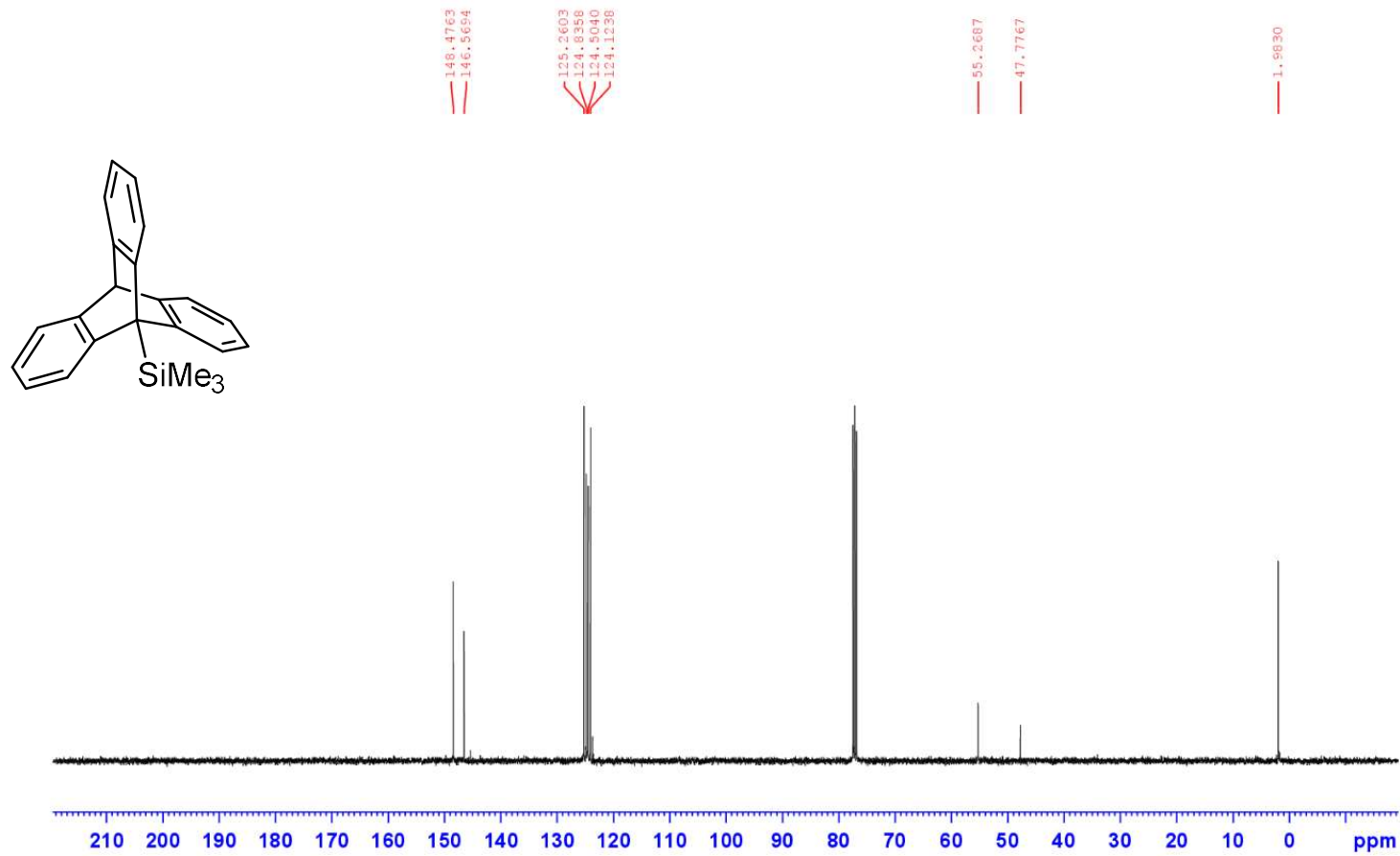
${ }^{1} \mathrm{H}$ NMR of Trip- $t$-Bu (400 MHz, $\left.\mathrm{CDCl}_{3}\right)$

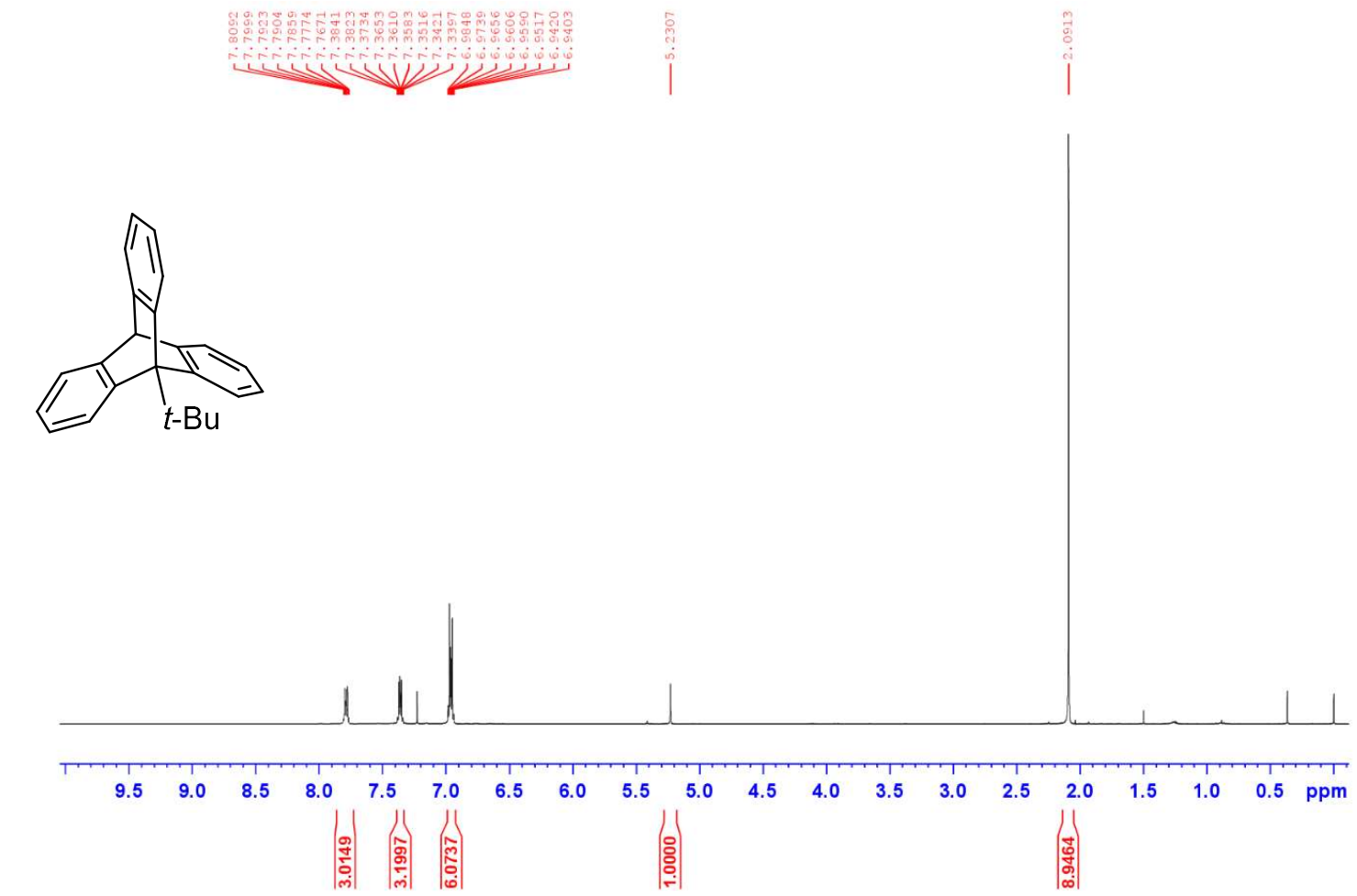

${ }^{13} \mathrm{C}$ NMR of Trip- $t$-Bu $\left(100 \mathrm{MHz}, \mathrm{CDCl}_{3}\right)$
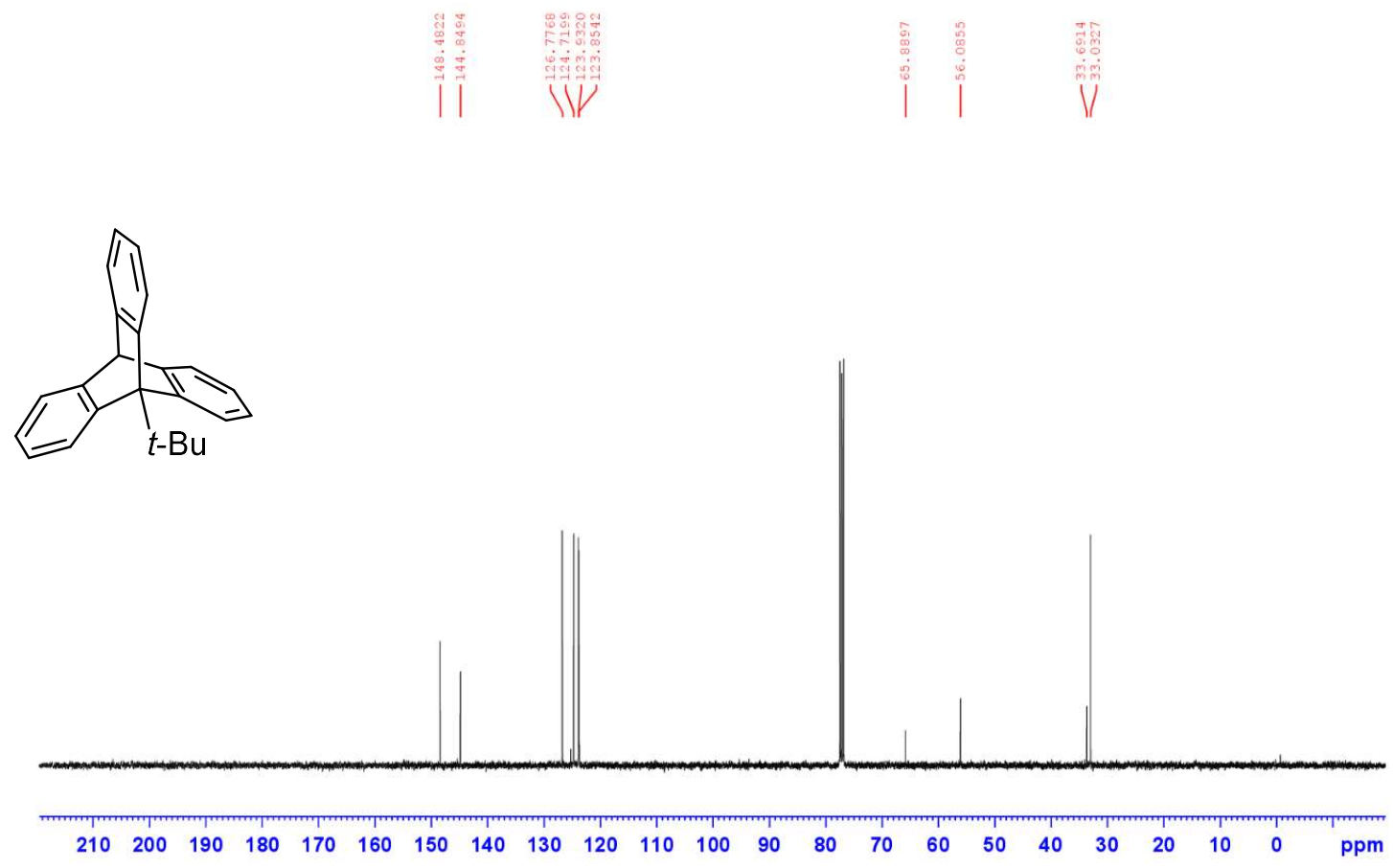
${ }^{1} \mathrm{H} \mathrm{NMR}$ of $2 \mathrm{e}\left(400 \mathrm{MHz}, \mathrm{CDCl}_{3}\right)$

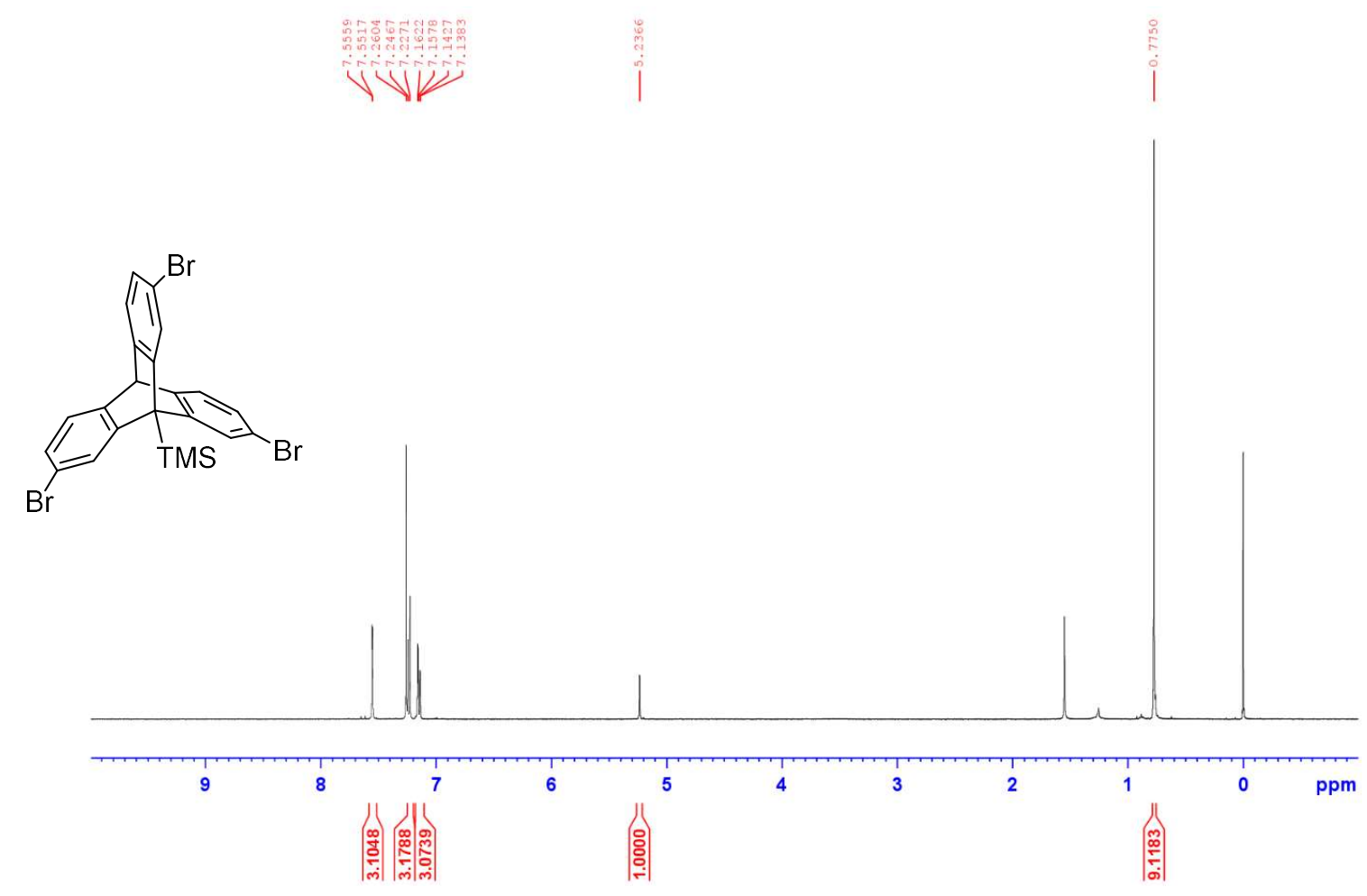

${ }^{13} \mathrm{C}$ NMR of $2 \mathrm{e}\left(100 \mathrm{MHz}, \mathrm{CDCl}_{3}\right)$
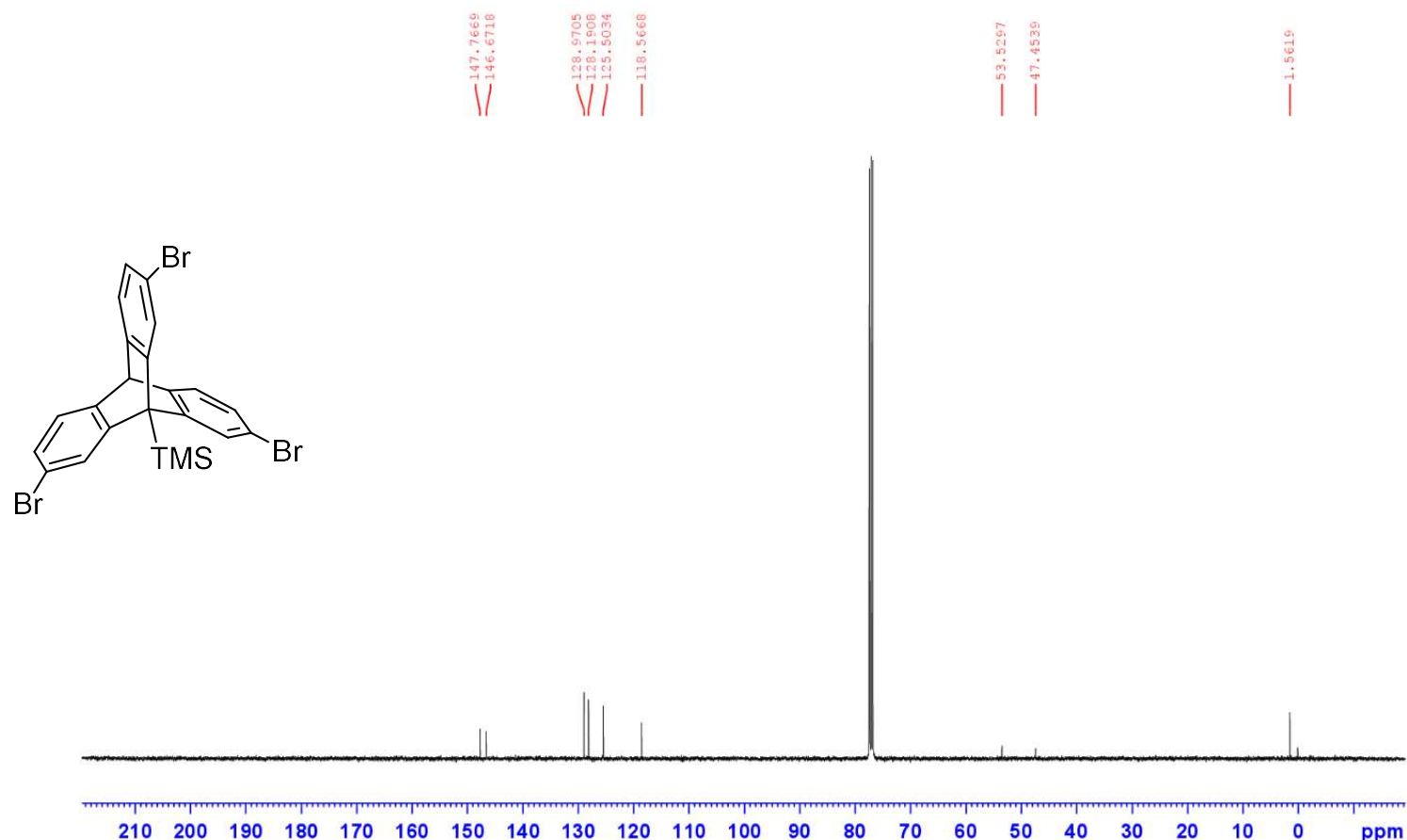
HSQC of $2 \mathbf{e}\left(\mathrm{CDCl}_{3}\right)$

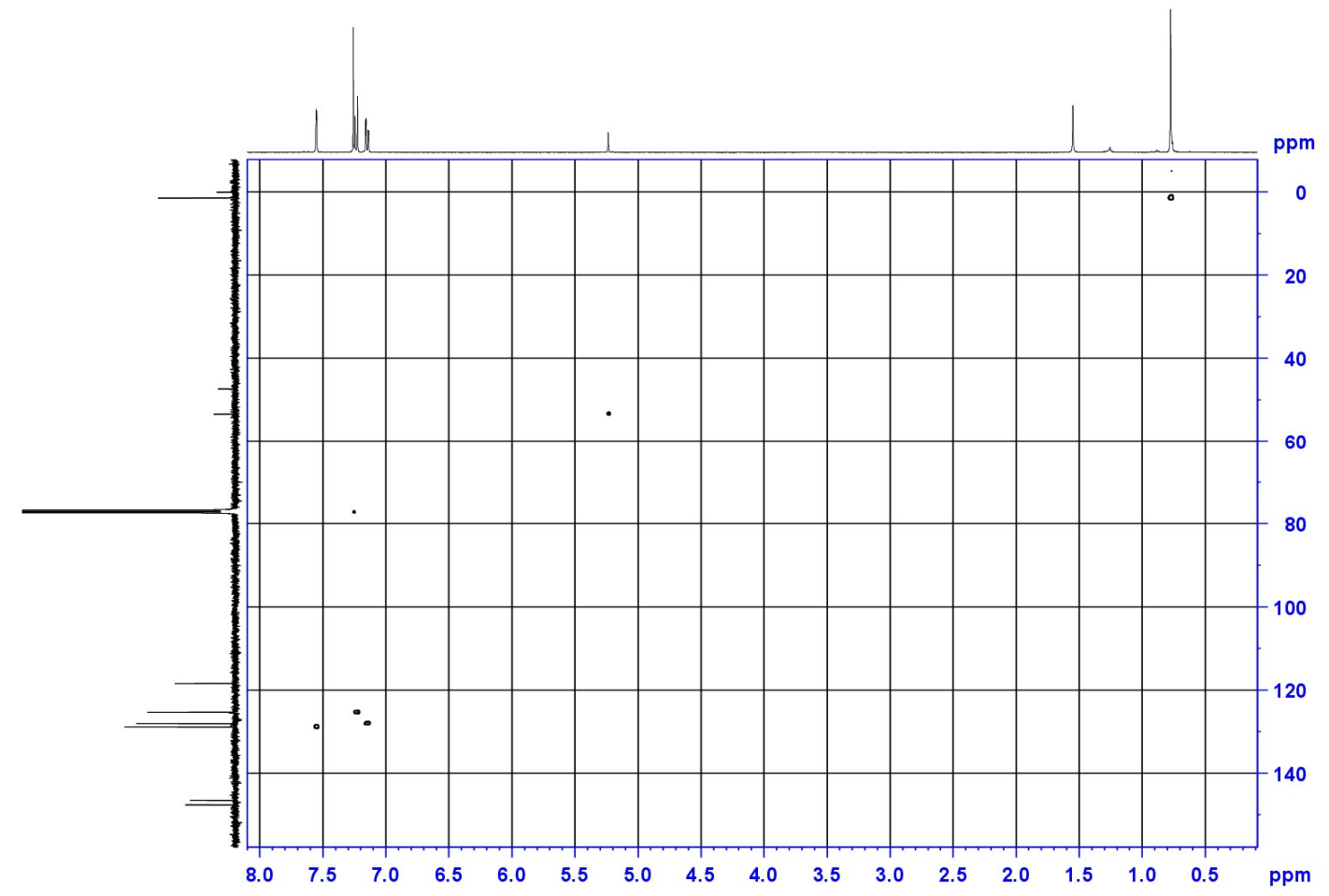

$\mathrm{HMBC}$ of $\mathbf{2 a}\left(\mathrm{CDCl}_{3}\right)$

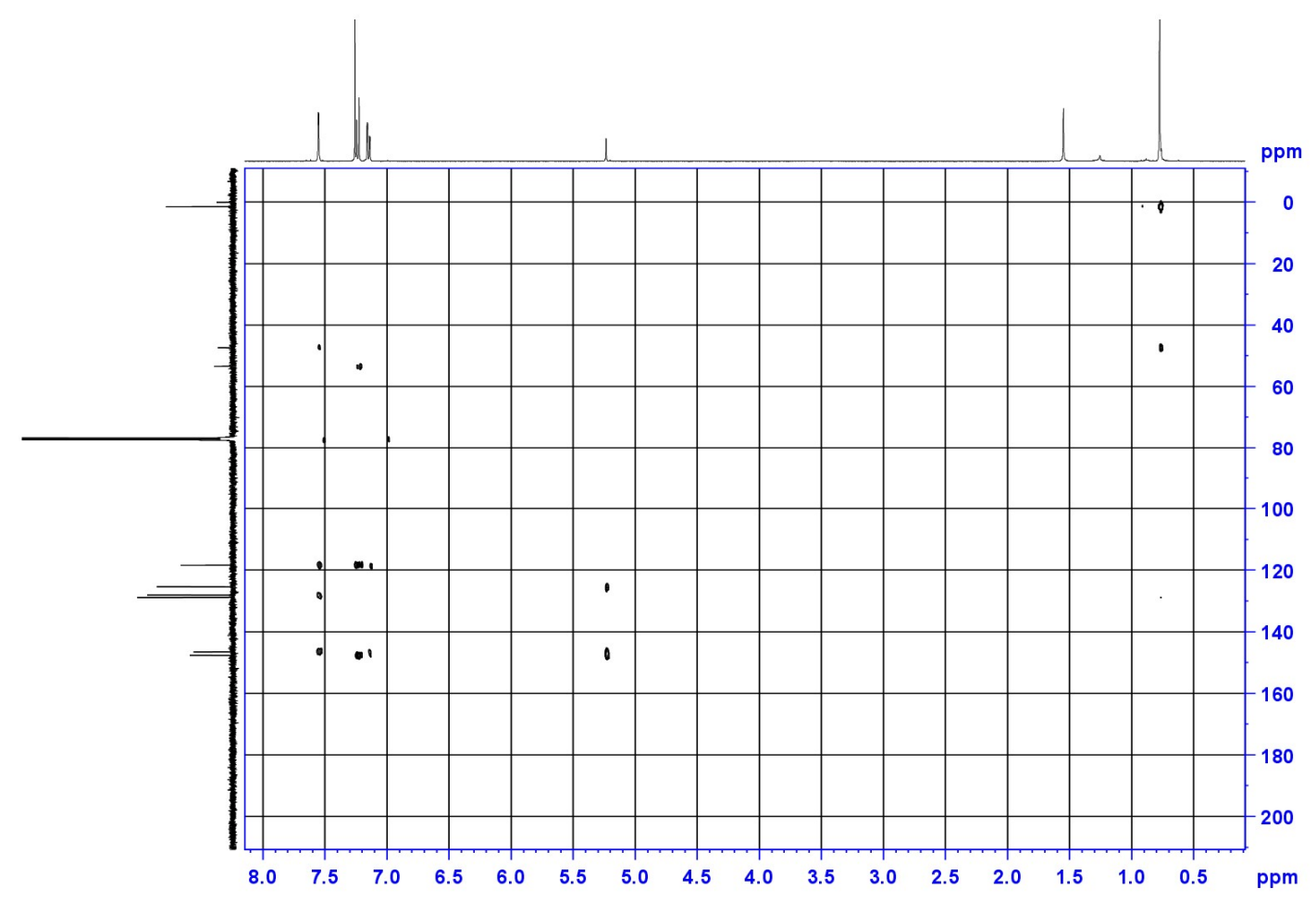


${ }^{1} \mathrm{H}$ NMR of $2 \mathbf{f}\left(400 \mathrm{MHz}, \mathrm{CDCl}_{3}\right)$

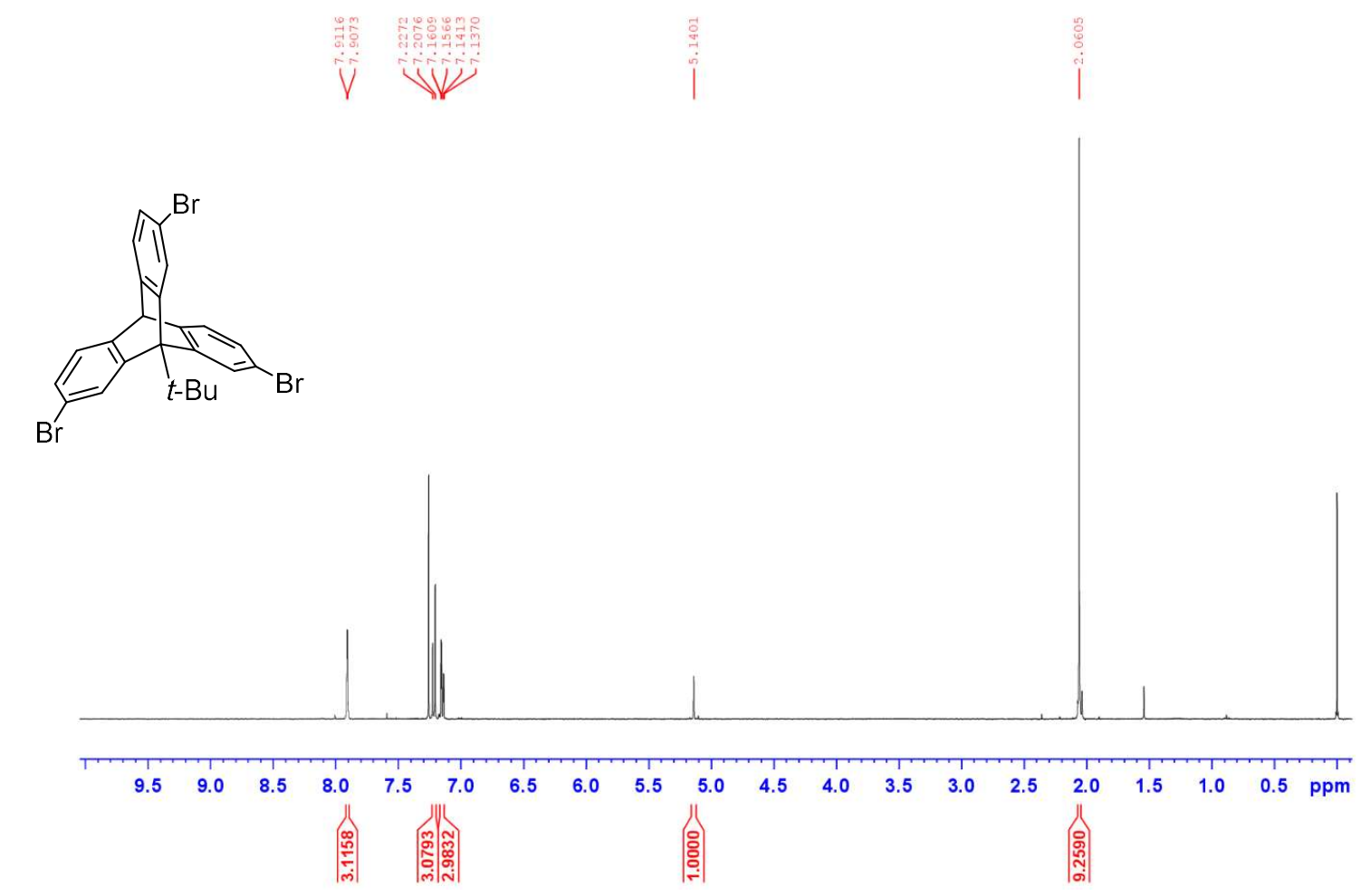

${ }^{13} \mathrm{C}$ NMR of $2 \mathbf{f}\left(100 \mathrm{MHz}, \mathrm{CDCl}_{3}\right)$

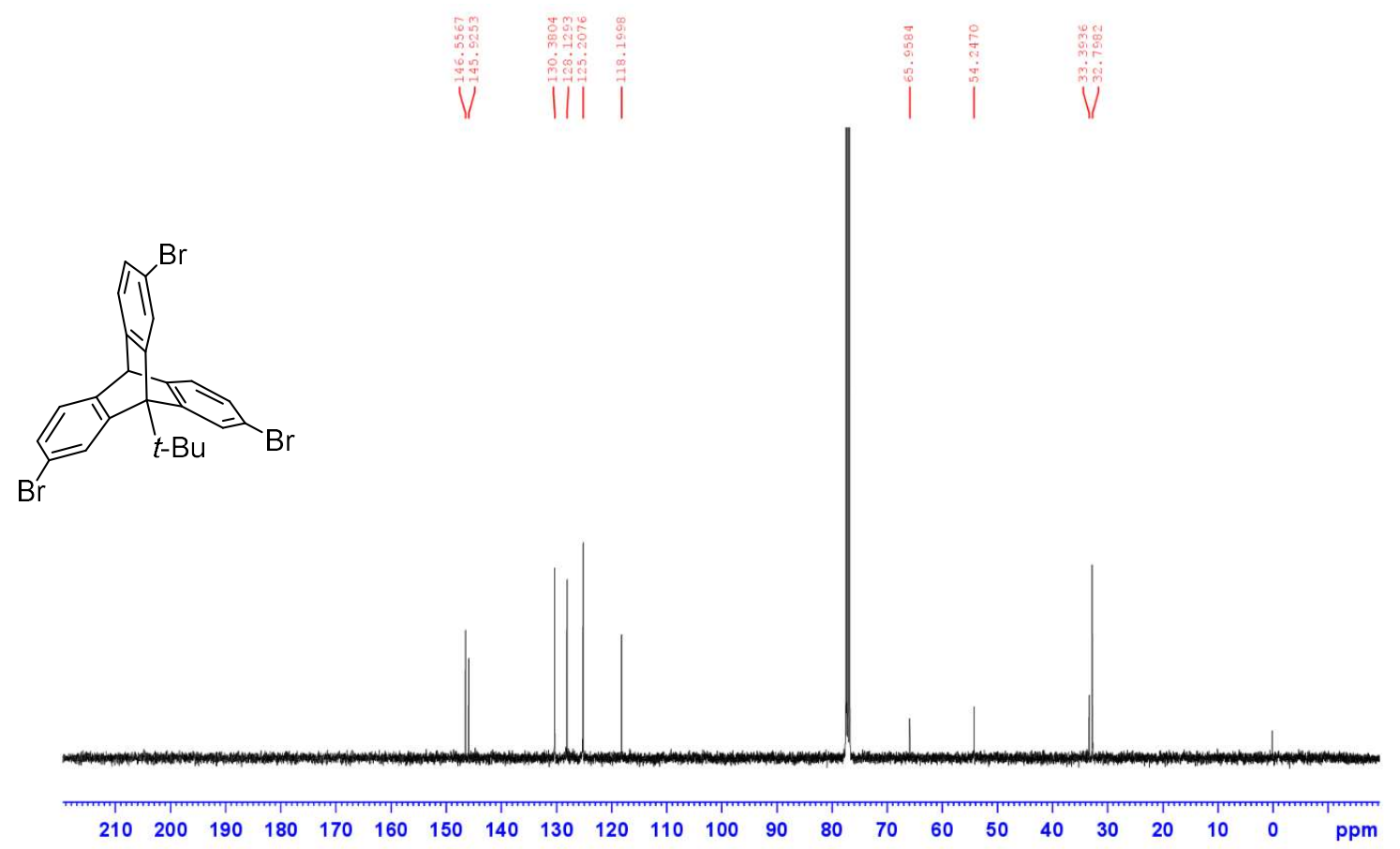


HSQC of $\mathbf{2 f}\left(\mathrm{CDCl}_{3}\right)$

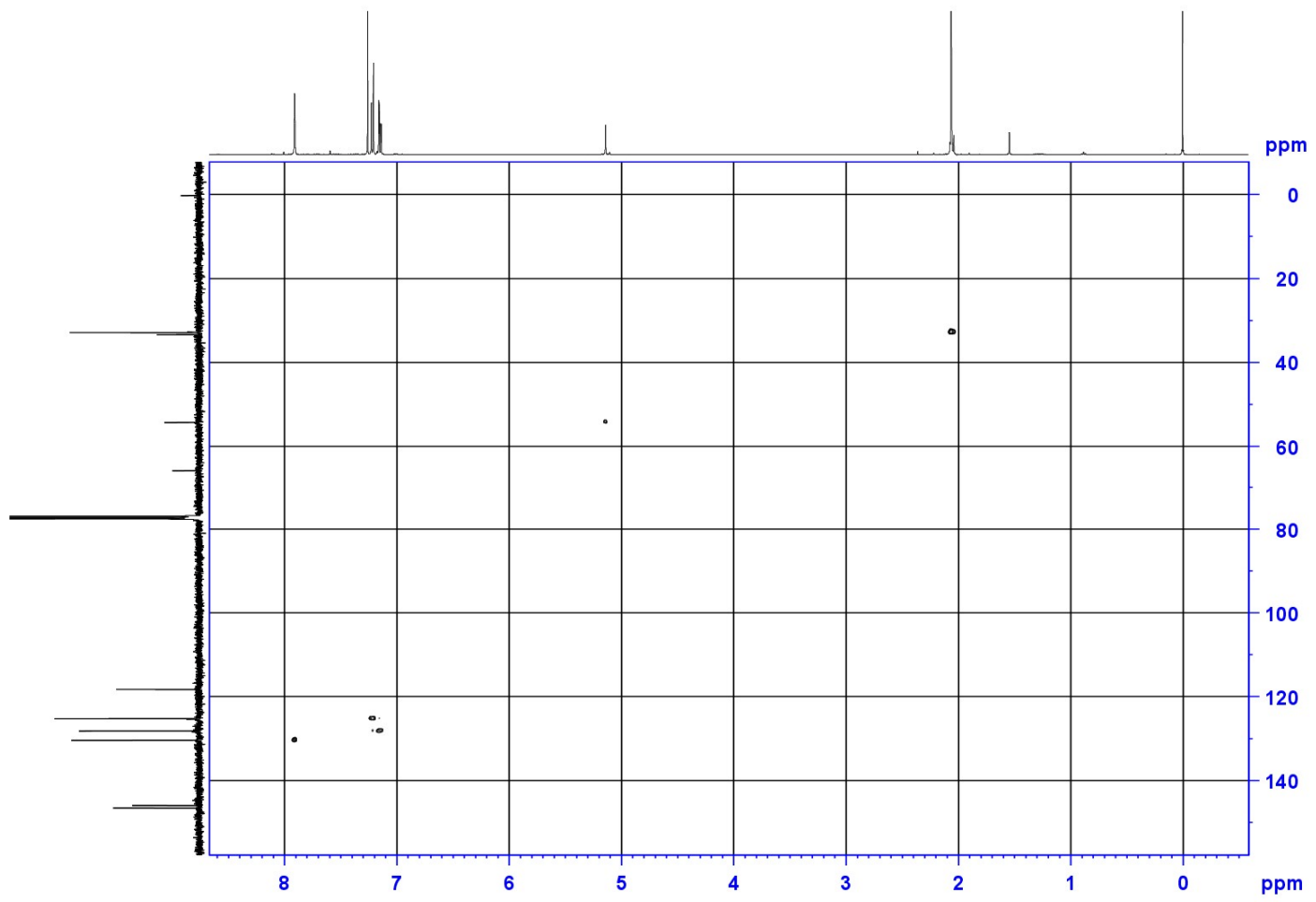

HMBC of $\mathbf{2} \mathbf{f}\left(\mathrm{CDCl}_{3}\right)$

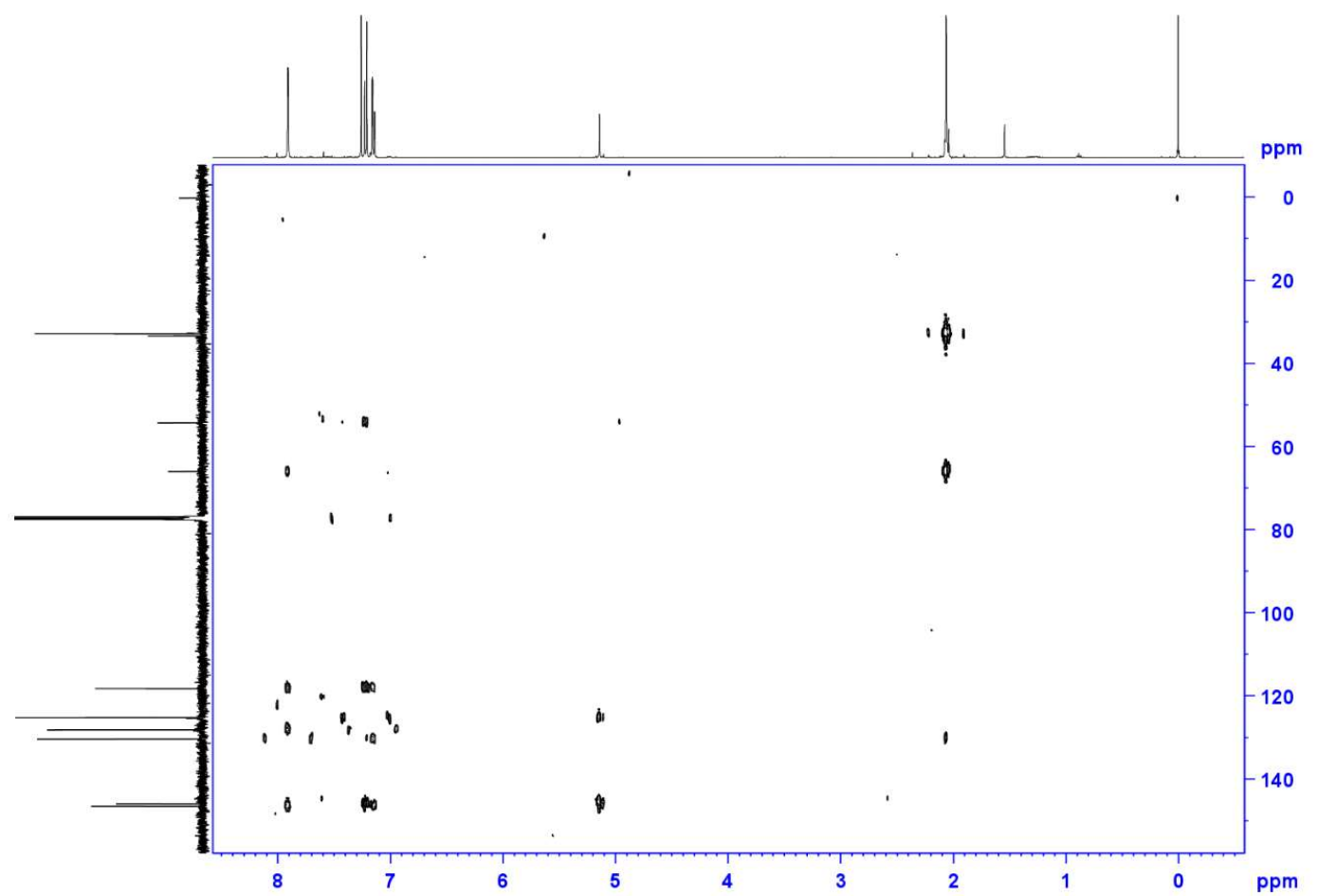


${ }^{1} \mathrm{H} \mathrm{NMR}$ of $4\left(400 \mathrm{MHz}, \mathrm{CDCl}_{3}\right)$

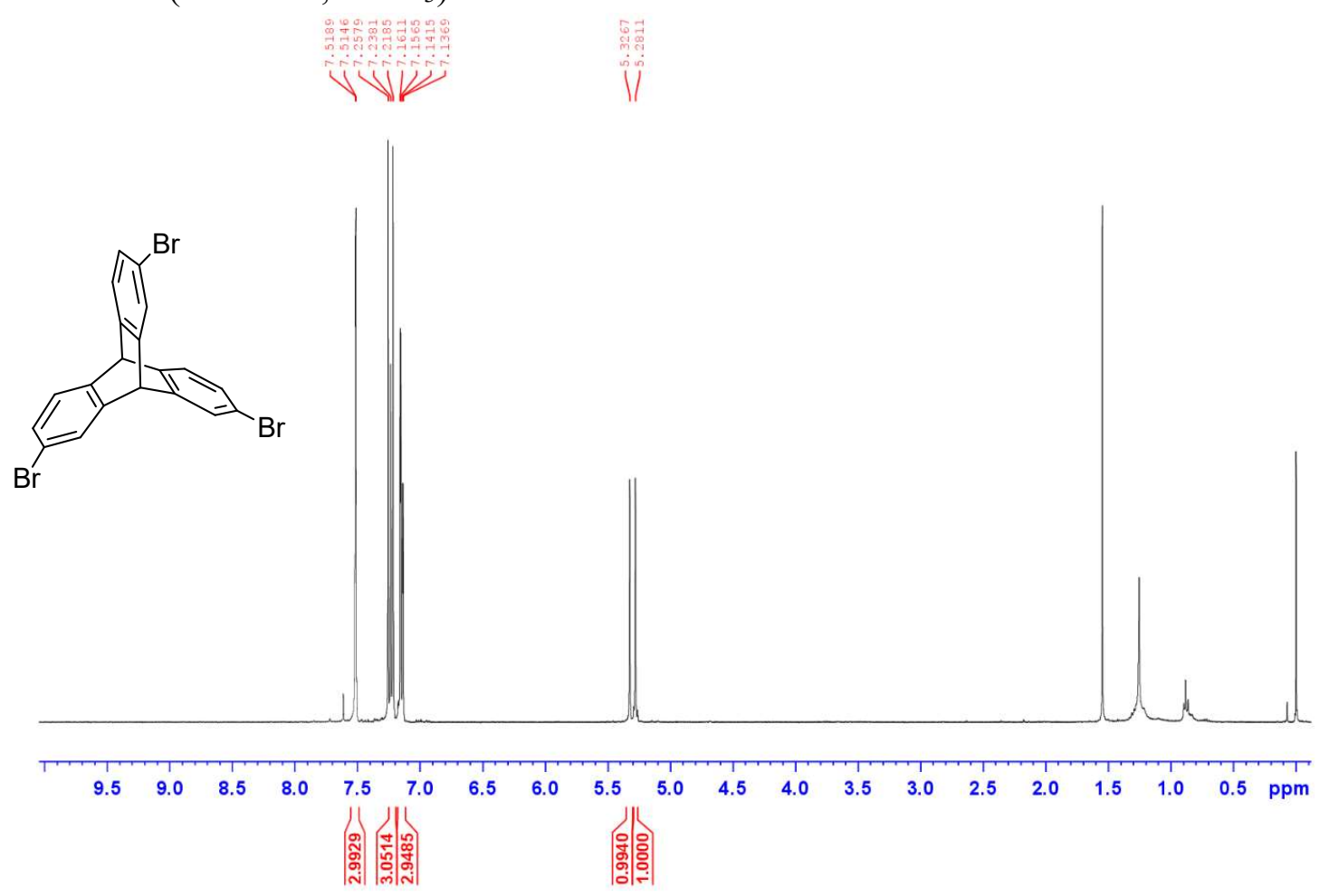

${ }^{13} \mathrm{C}$ NMR of $4\left(100 \mathrm{MHz}, \mathrm{CDCl}_{3}\right)$
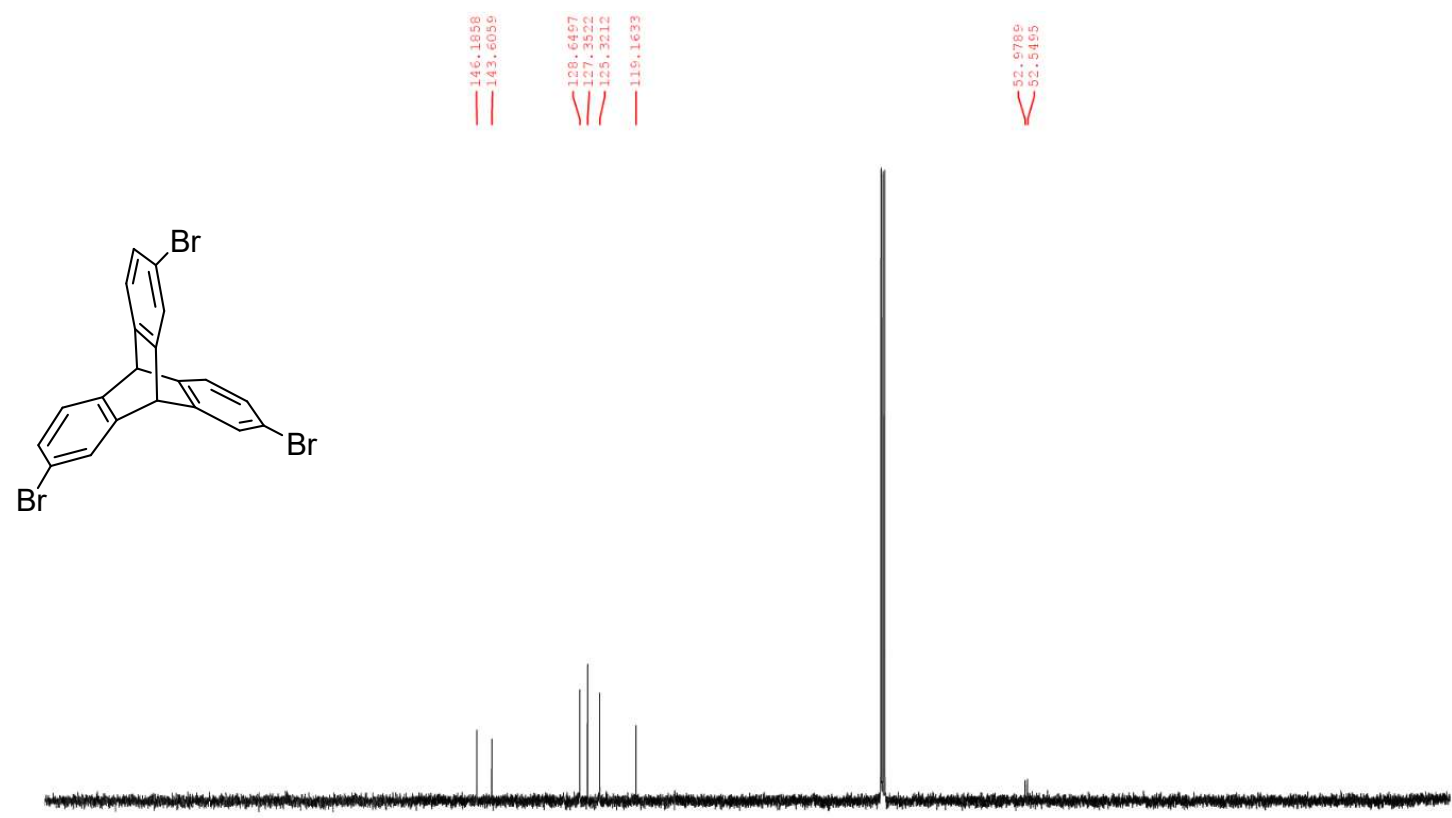

$\begin{array}{lllllllllllllllllllllll}210 & 200 & 190 & 180 & 170 & 160 & 150 & 140 & 130 & 120 & 110 & 100 & 90 & 80 & 70 & 60 & 50 & 40 & 30 & 20 & 10 & 0 & \mathrm{ppm}\end{array}$ 
${ }^{1} \mathrm{H}$ NMR of $5\left(400 \mathrm{MHz}, \mathrm{CDCl}_{3}\right)$

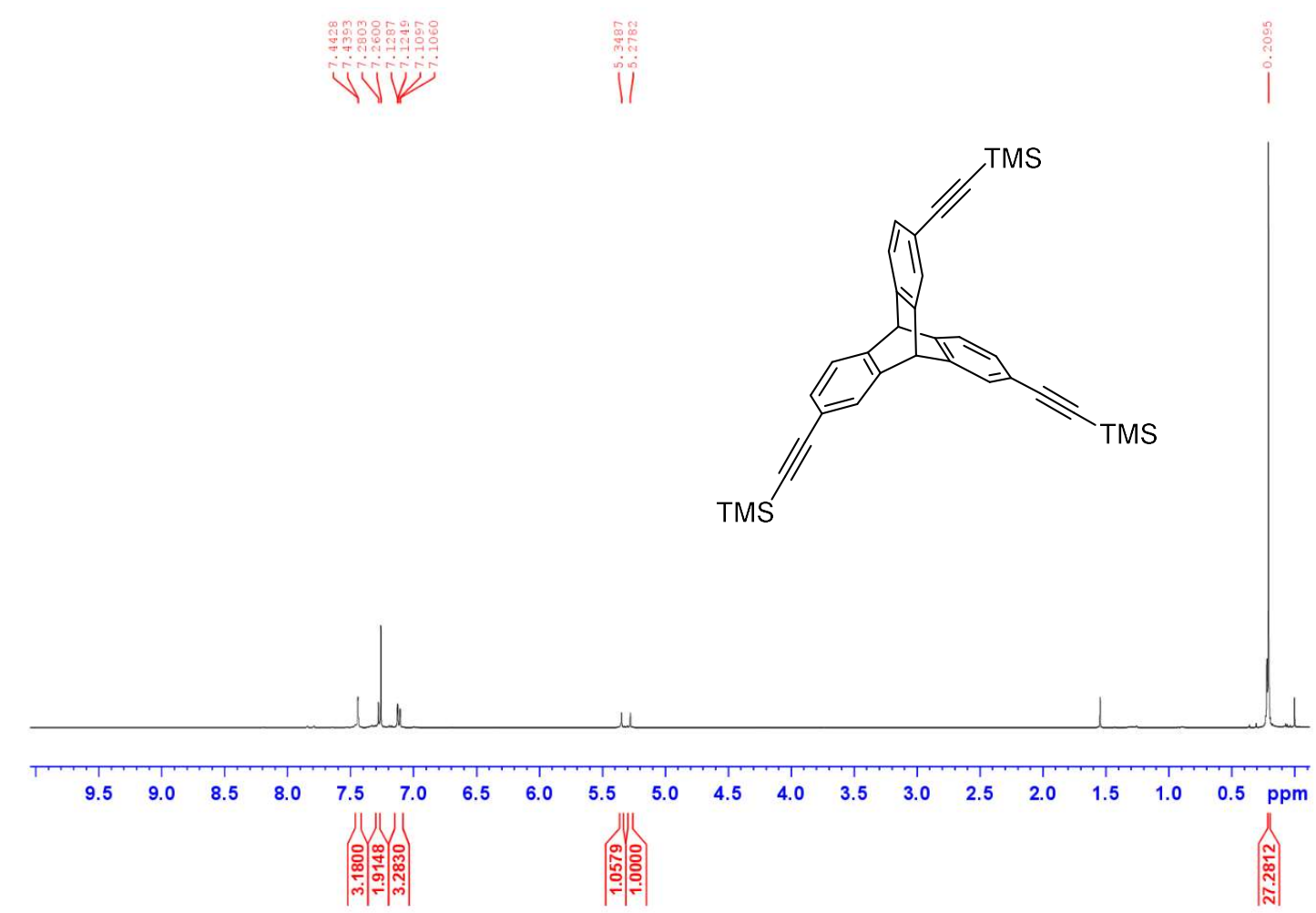

${ }^{13} \mathrm{C}$ NMR of $5\left(100 \mathrm{MHz}, \mathrm{CDCl}_{3}\right)$

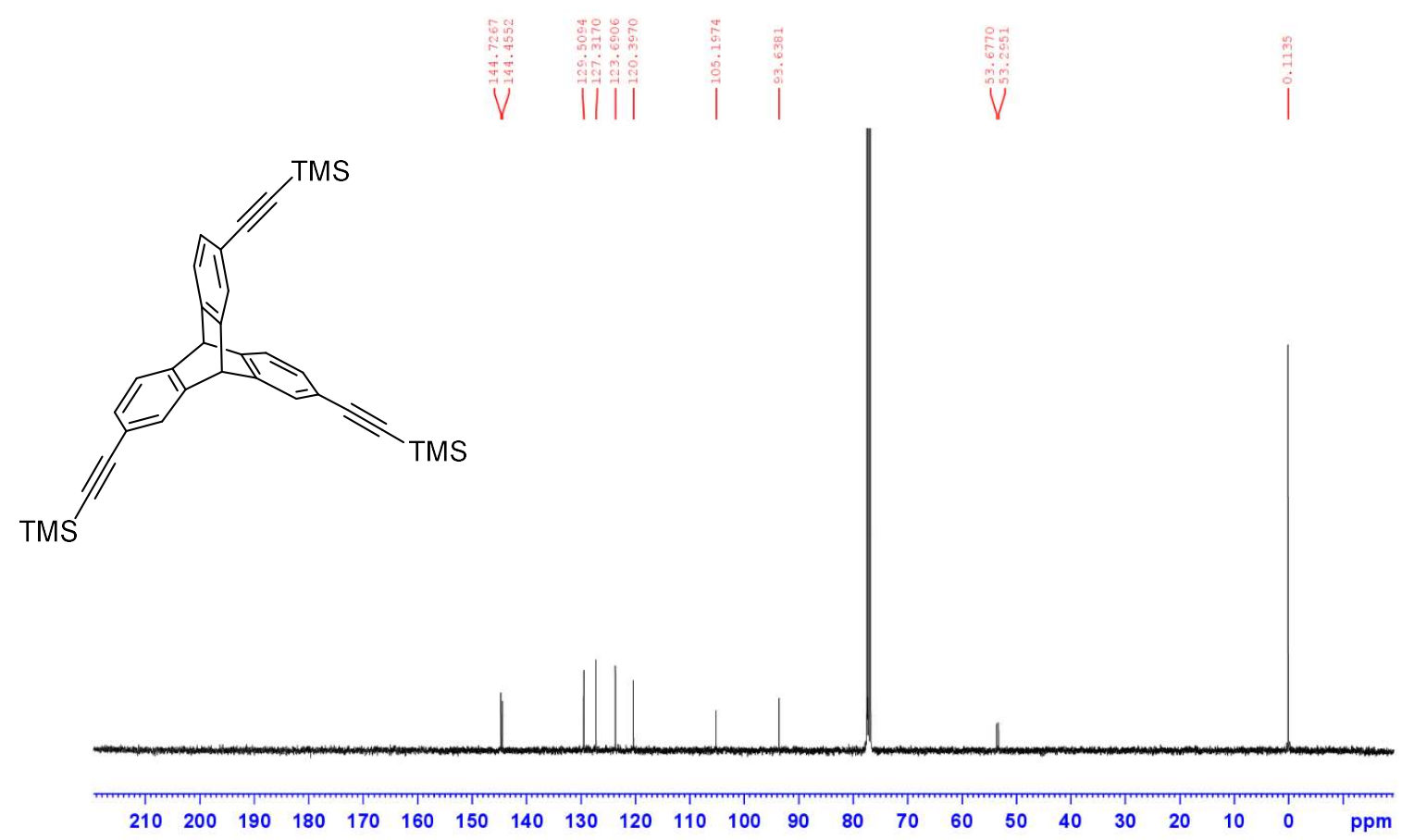


${ }^{1} \mathrm{H}$ NMR of $6\left(400 \mathrm{MHz}, \mathrm{CDCl}_{3}\right.$ )

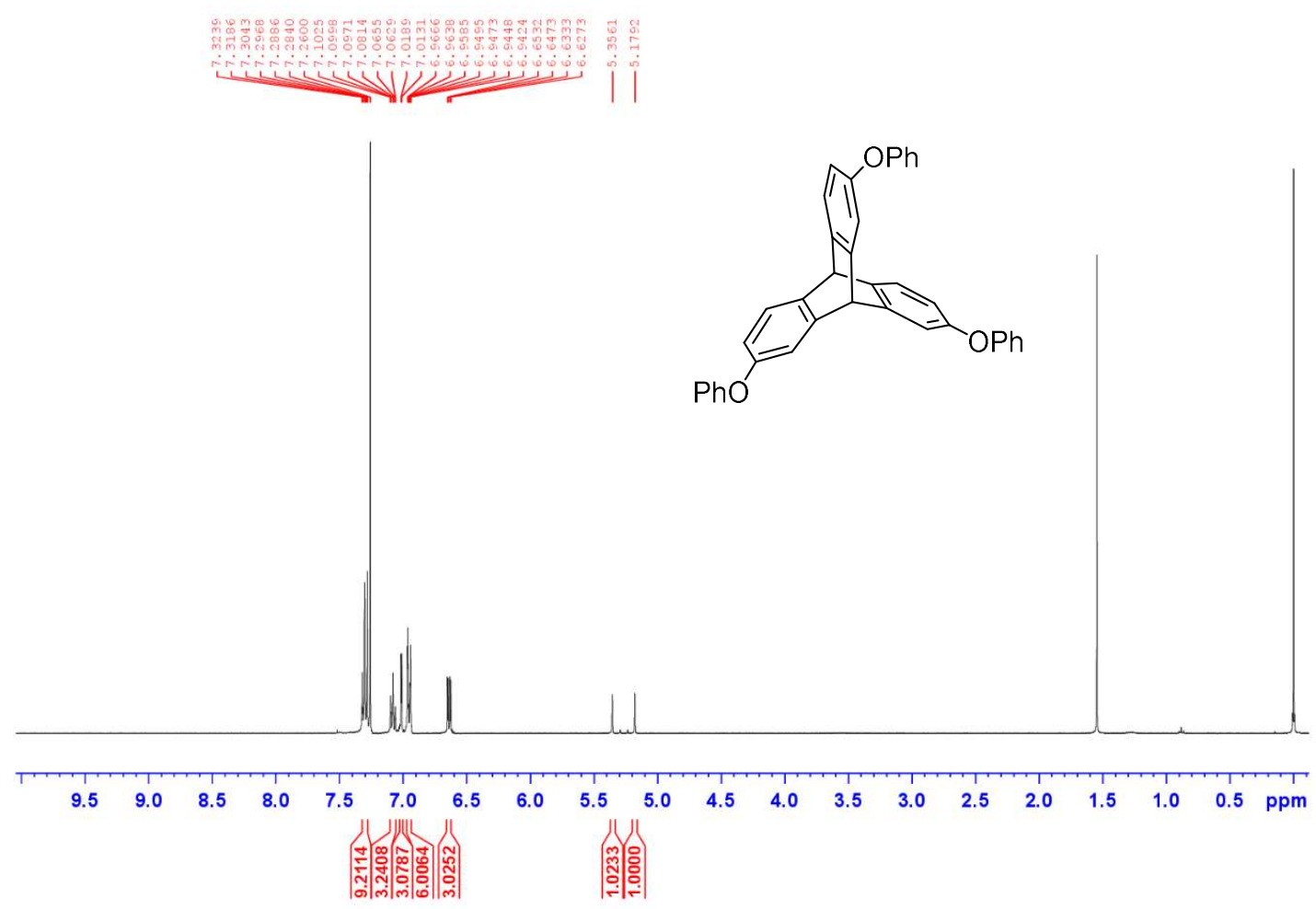

${ }^{13} \mathrm{C}$ NMR of $6\left(100 \mathrm{MHz}, \mathrm{CDCl}_{3}\right)$

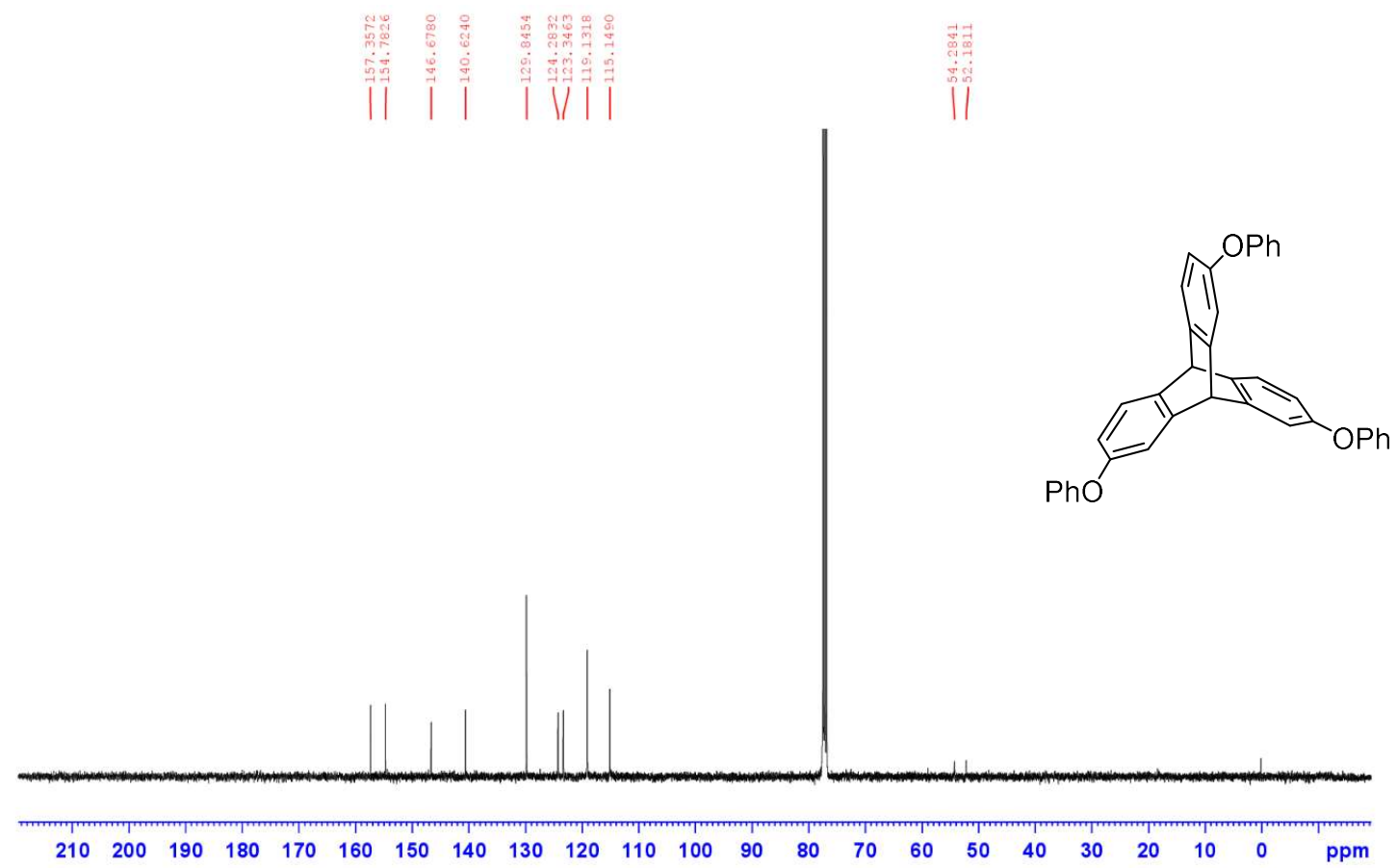


${ }^{1} \mathrm{H} \mathrm{NMR}$ of $7\left(400 \mathrm{MHz}, \mathrm{CDCl}_{3}\right)$

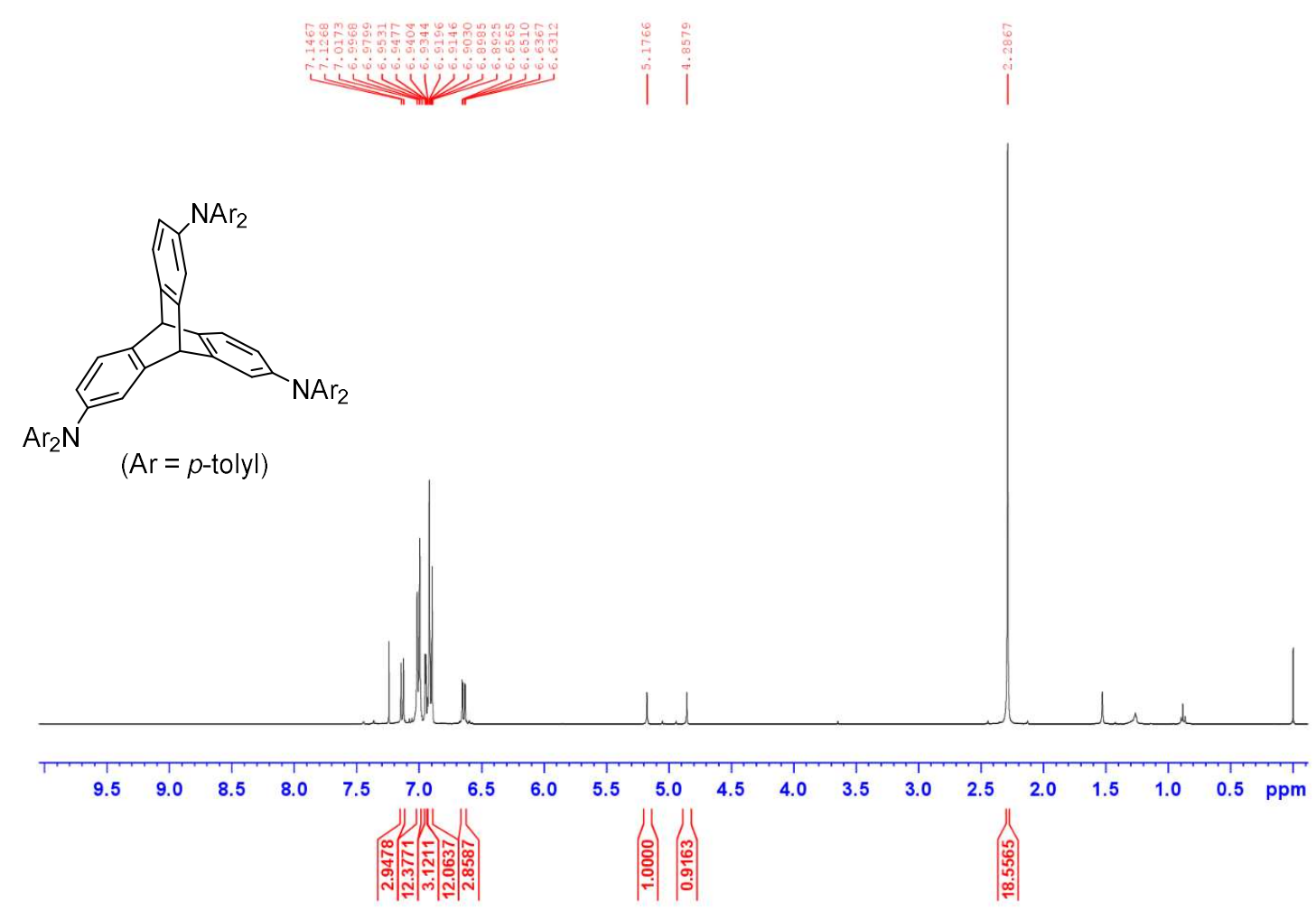

${ }^{13} \mathrm{C}$ NMR of $7\left(100 \mathrm{MHz}, \mathrm{CDCl}_{3}\right)$
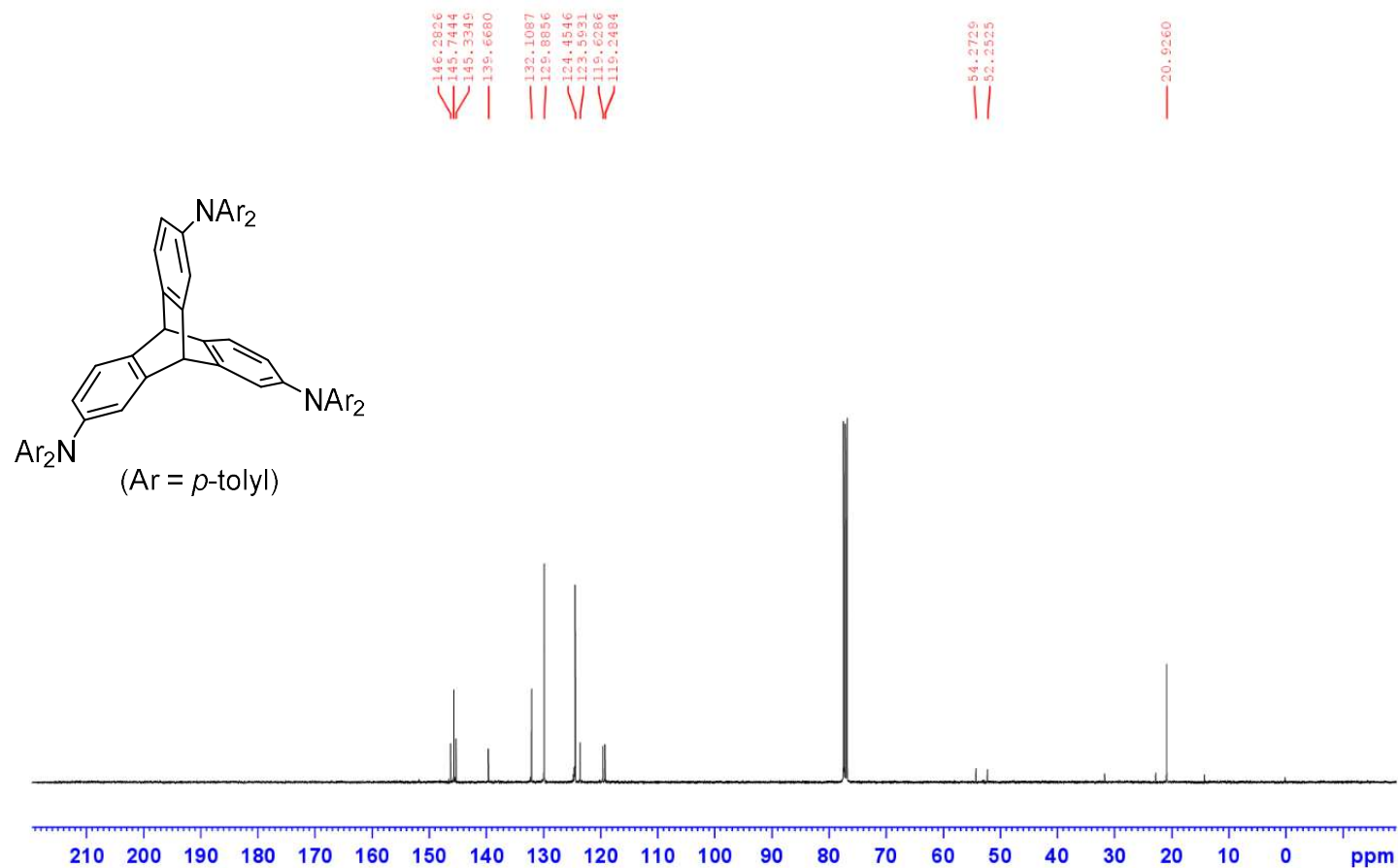
${ }^{1} \mathrm{H}$ NMR of $8\left(400 \mathrm{MHz}, \mathrm{CDCl}_{3}\right)$

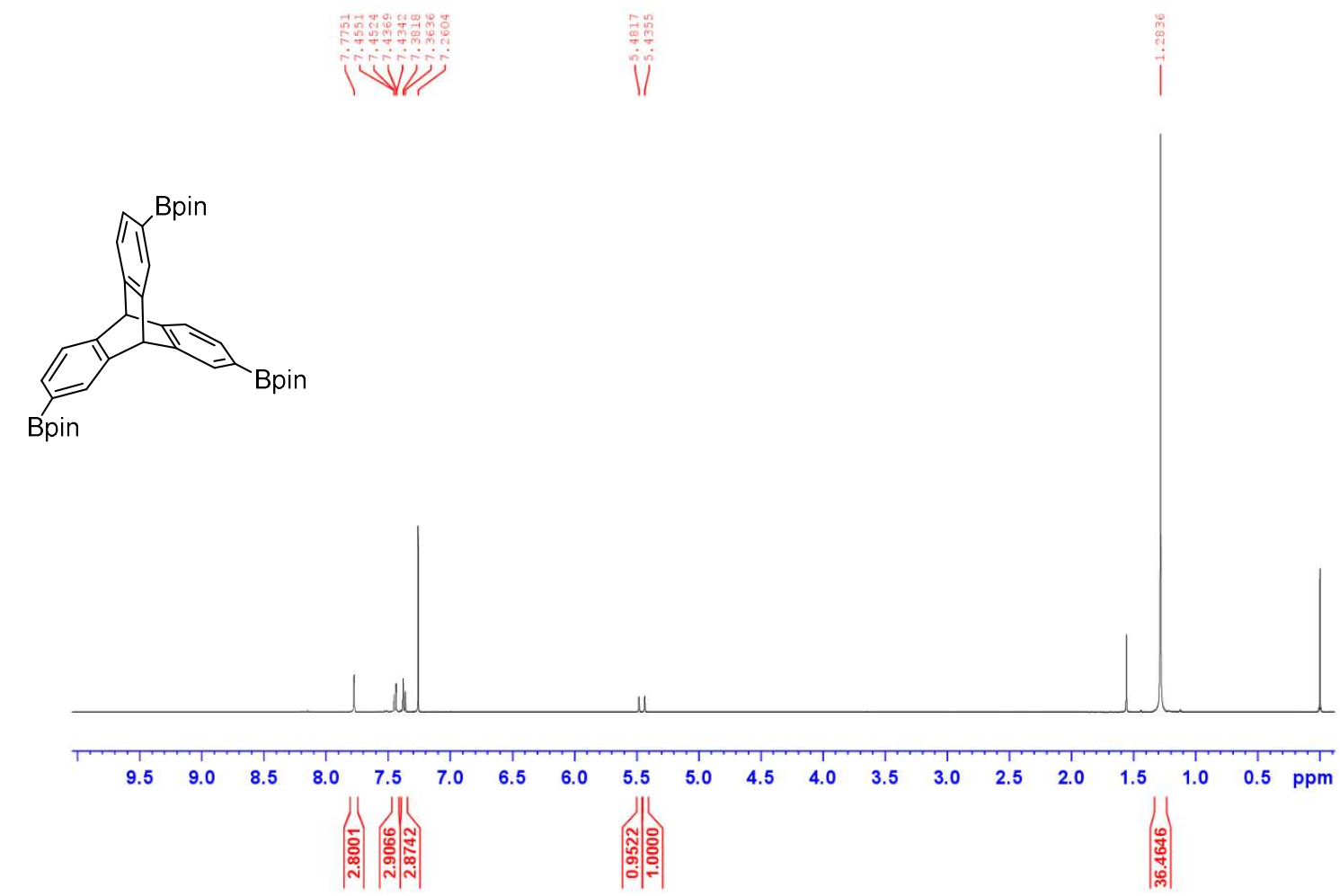

${ }^{13} \mathrm{C} \mathrm{NMR}$ of $8\left(100 \mathrm{MHz}, \mathrm{CDCl}_{3}\right)$
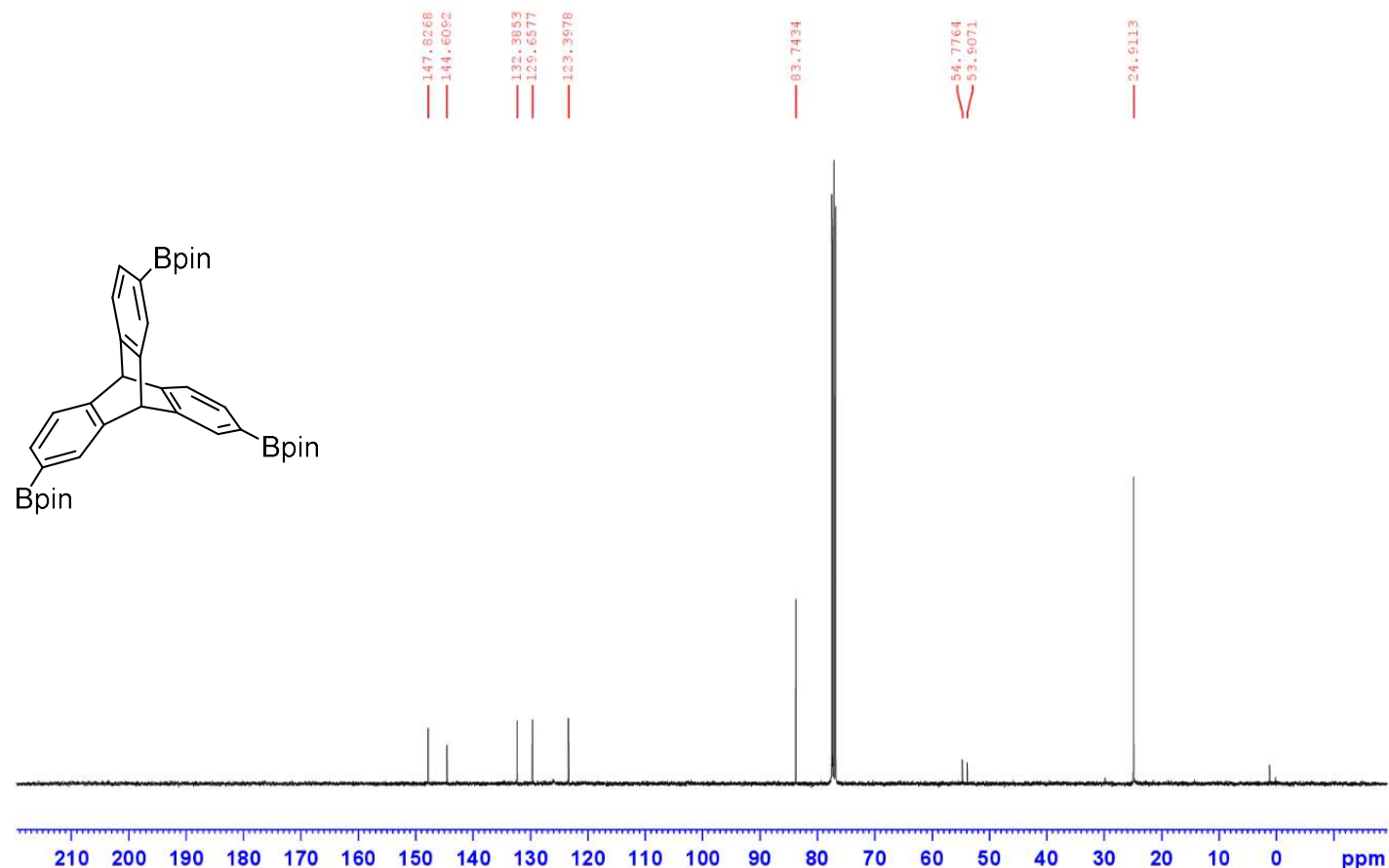
${ }^{1} \mathrm{H}$ NMR of $9\left(400 \mathrm{MHz}, \mathrm{CDCl}_{3}\right)$

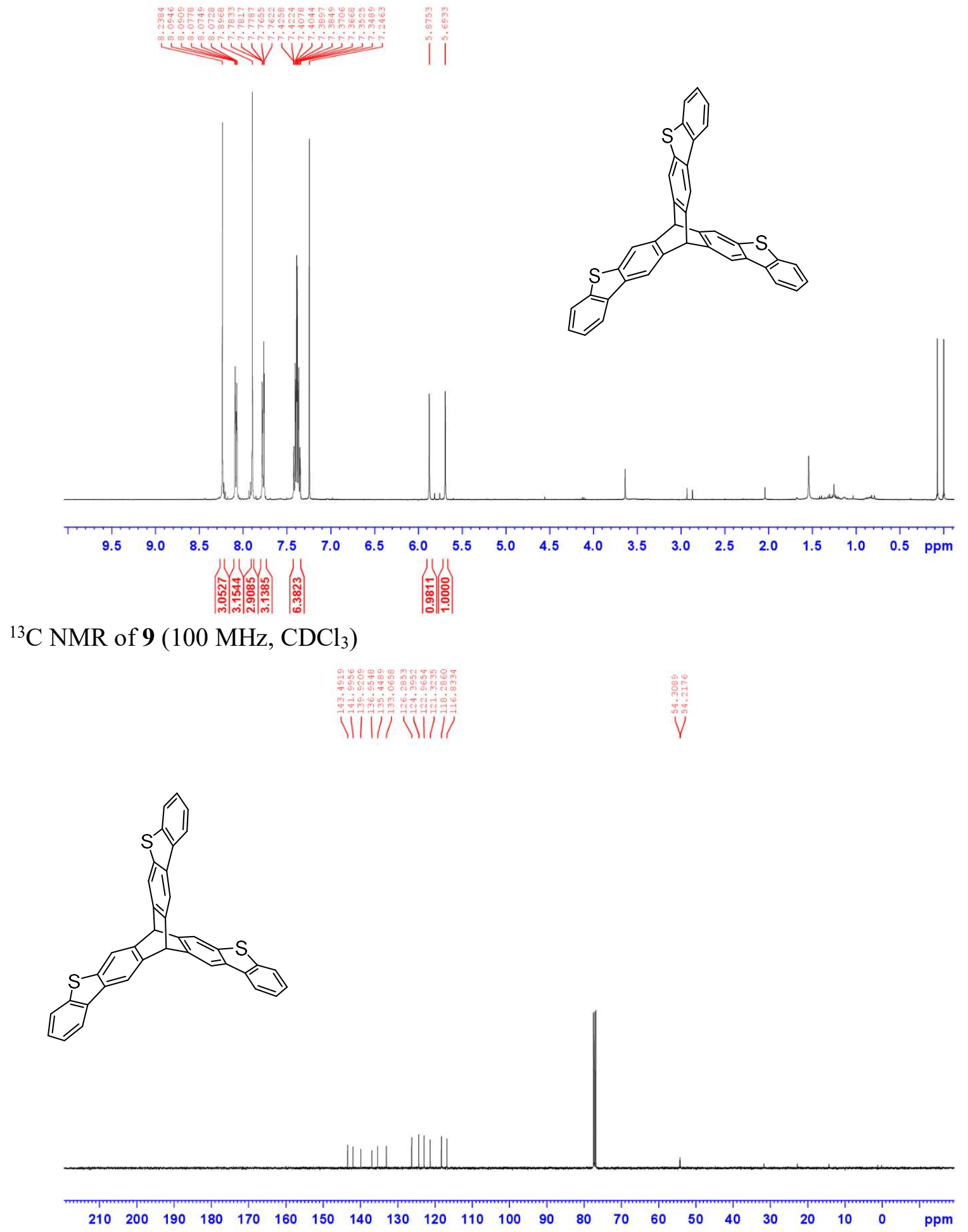


${ }^{1} \mathrm{H} \mathrm{NMR}$ of $10\left(400 \mathrm{MHz}, \mathrm{CDCl}_{3}\right)$
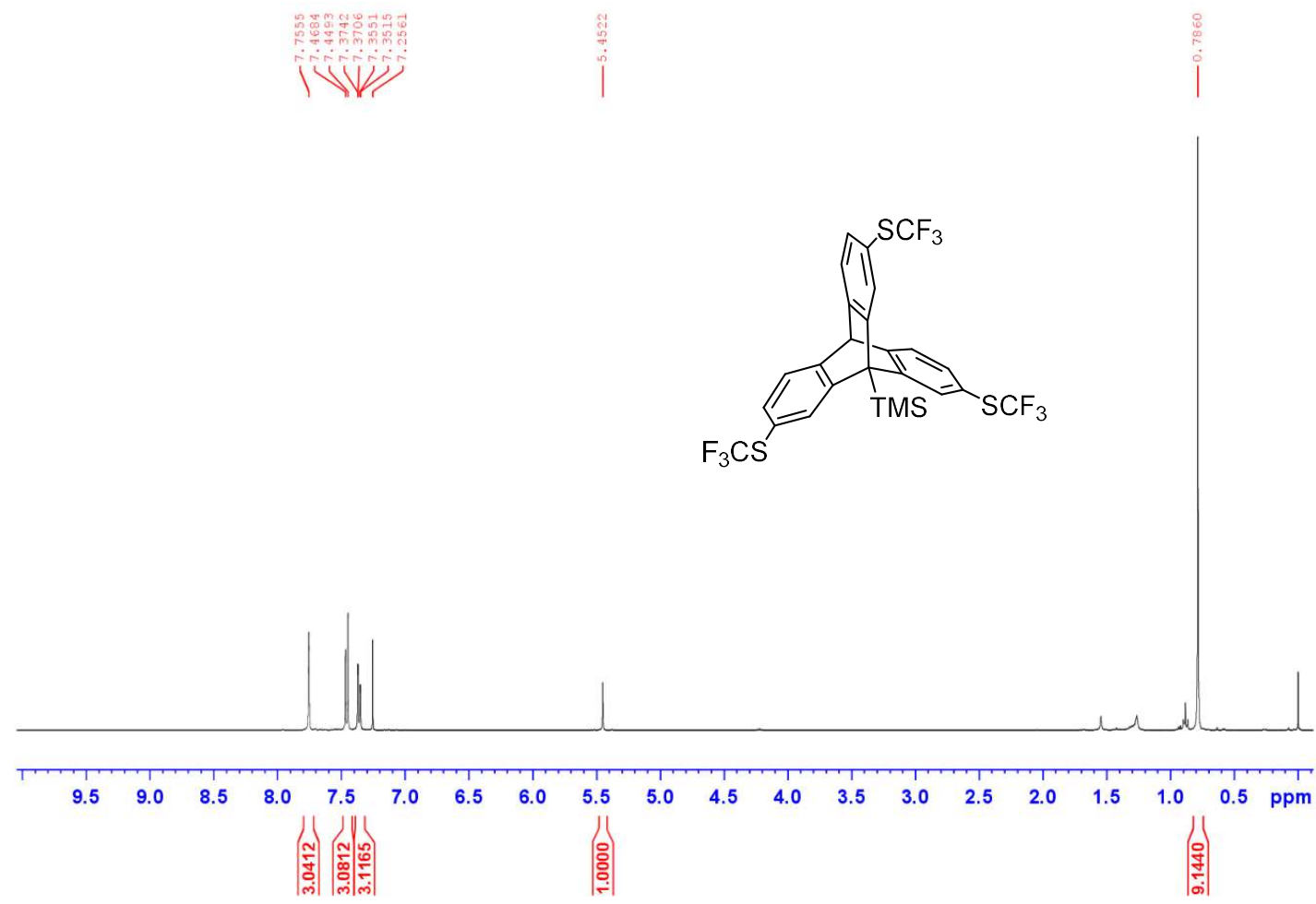

${ }^{13} \mathrm{C}$ NMR of $10\left(100 \mathrm{MHz}, \mathrm{CDCl}_{3}\right)$
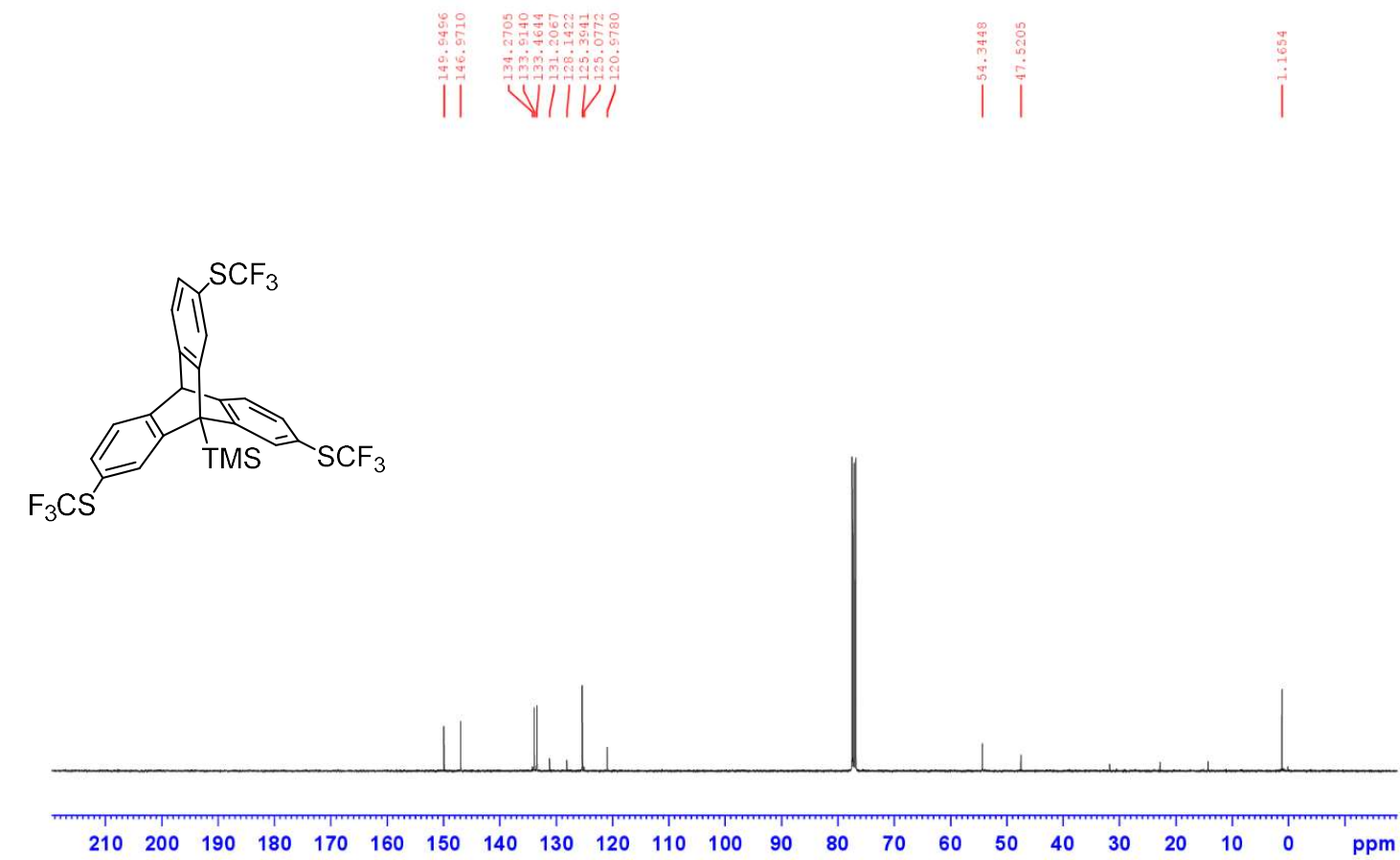
${ }^{19} \mathrm{~F}$ NMR of $10\left(376 \mathrm{MHz}, \mathrm{CDCl}_{3}\right)$

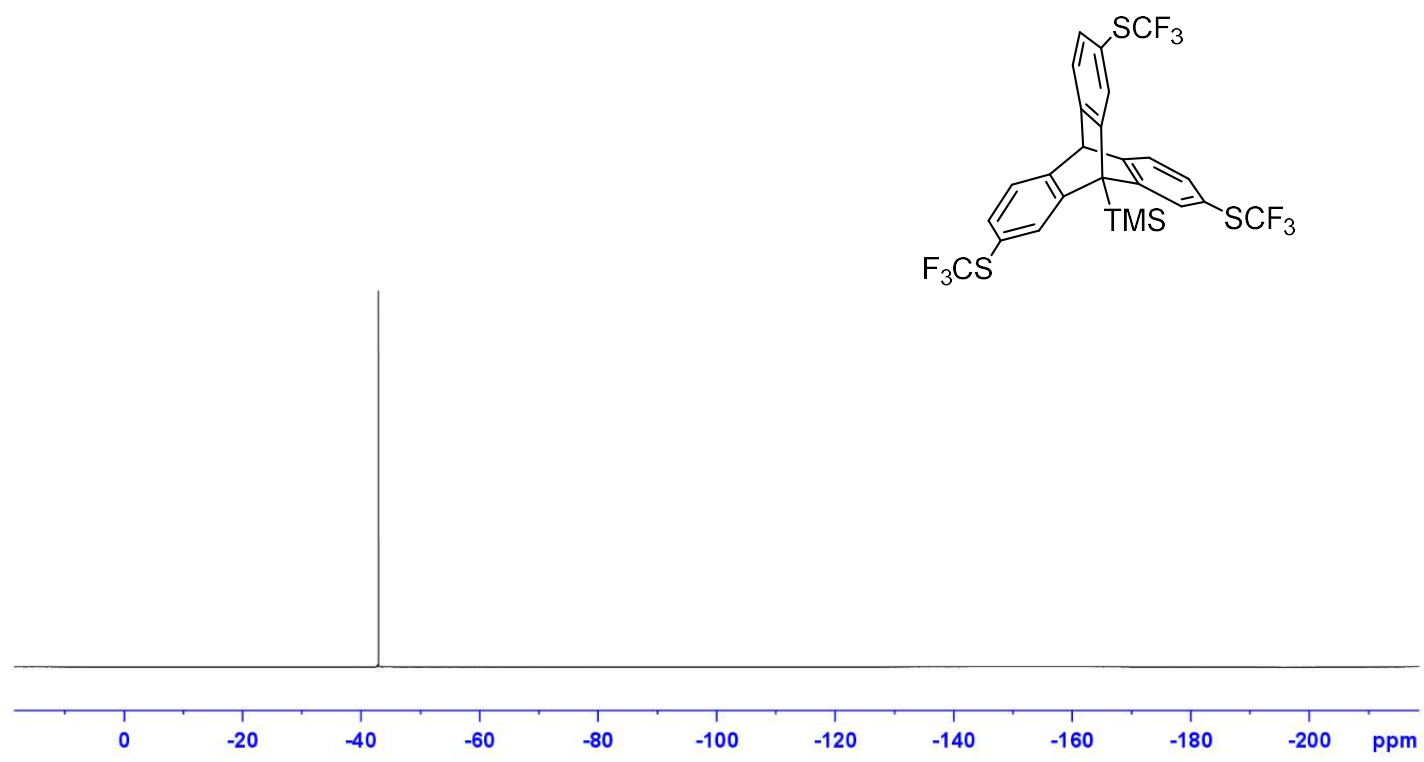

HSQC of $10\left(\mathrm{CDCl}_{3}\right)$

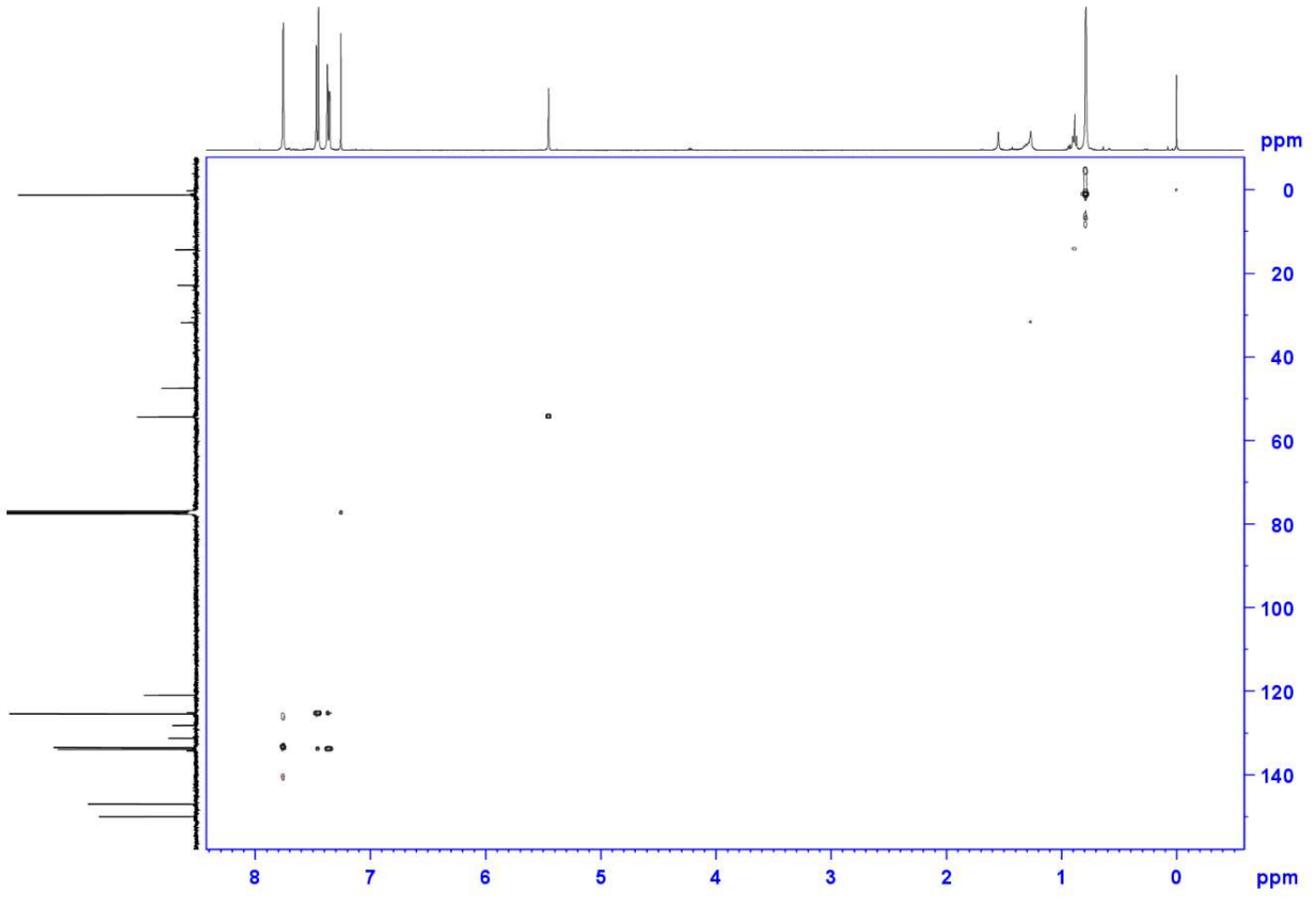


$\mathrm{HMBC}$ of $\mathbf{1 0}\left(\mathrm{CDCl}_{3}\right)$

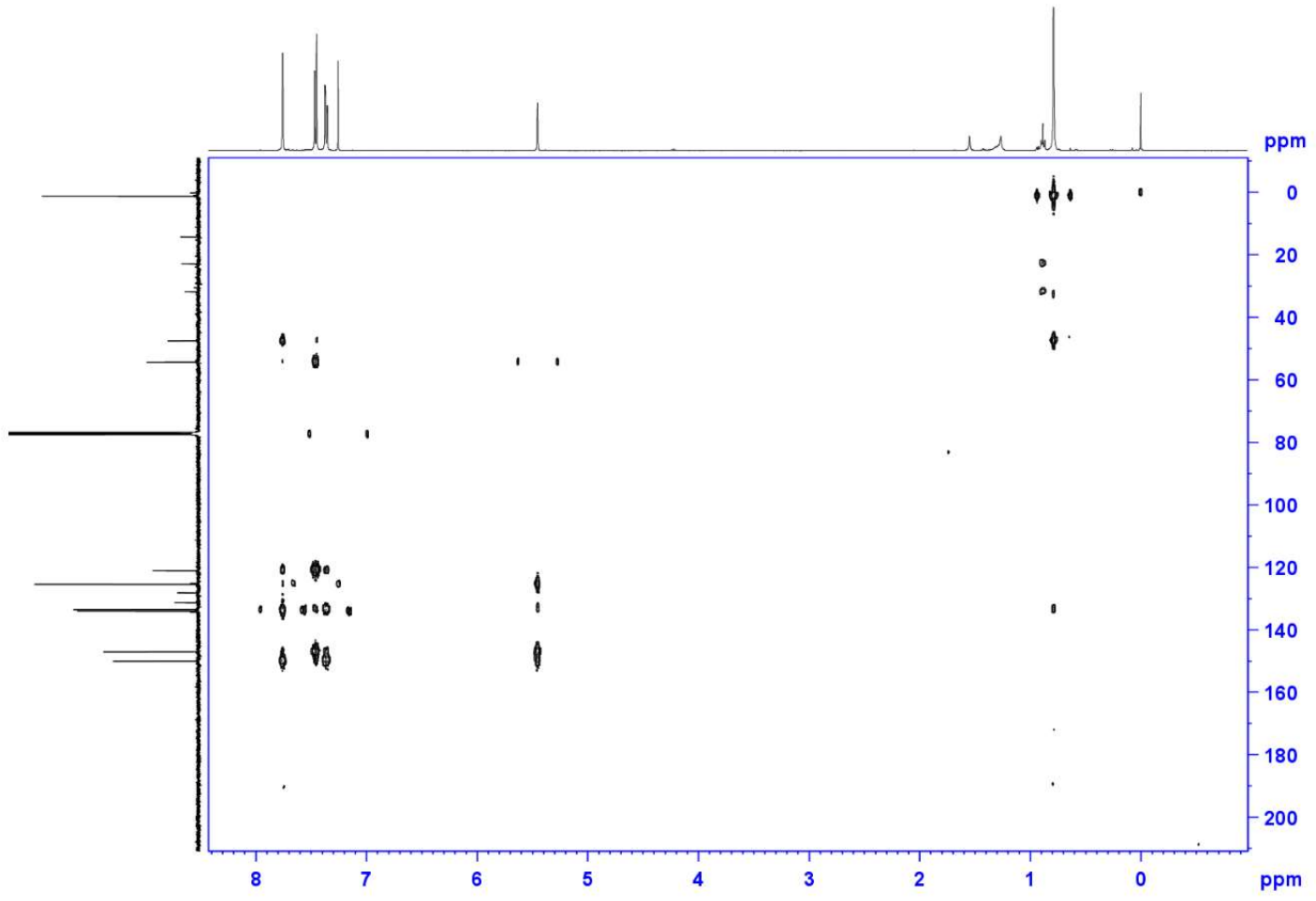


${ }^{1} \mathrm{H} \mathrm{NMR}$ of $11\left(400 \mathrm{MHz}, \mathrm{CDCl}_{3}\right)$
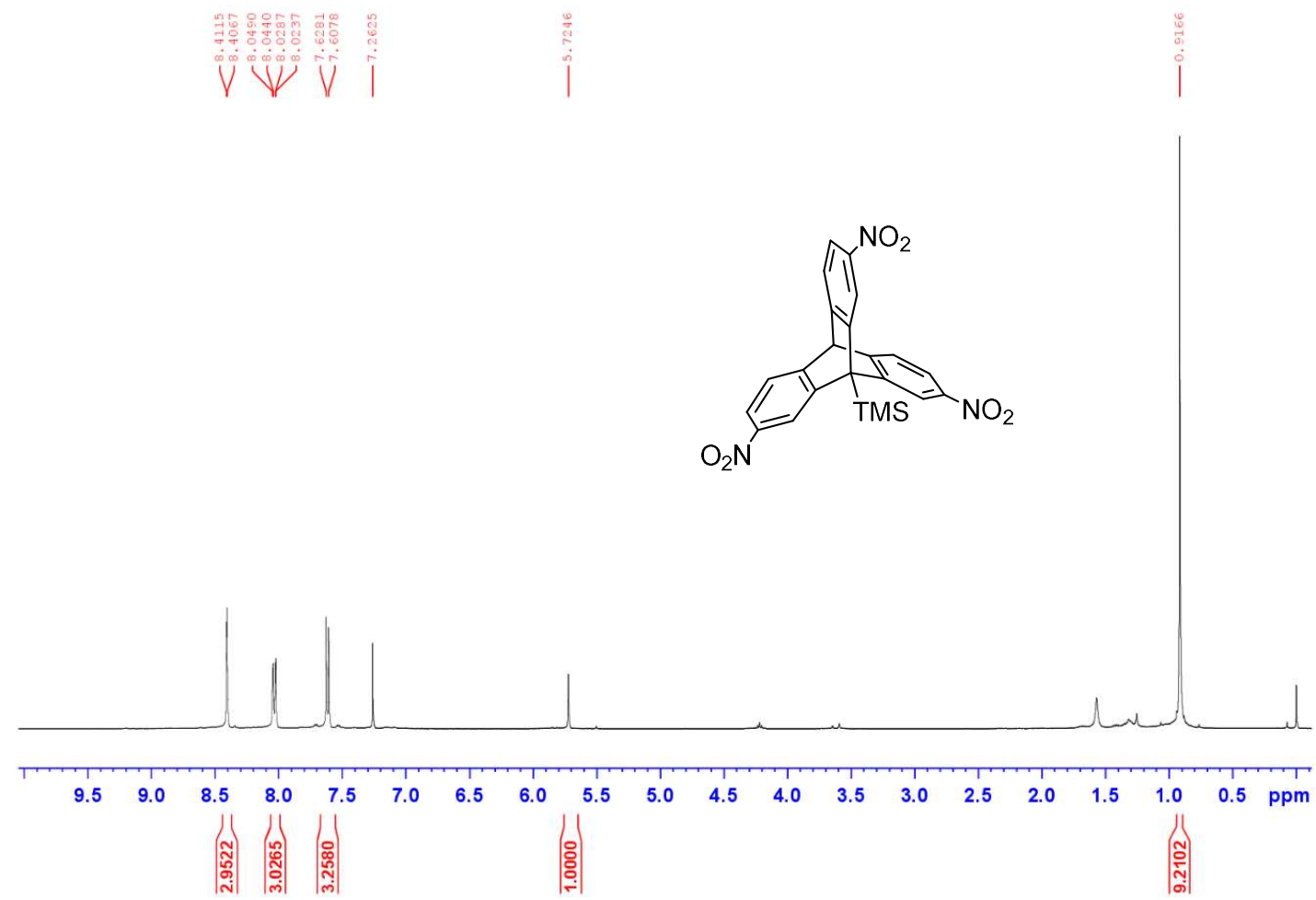

${ }^{13} \mathrm{C}$ NMR of $11\left(100 \mathrm{MHz}, \mathrm{CDCl}_{3}\right)$

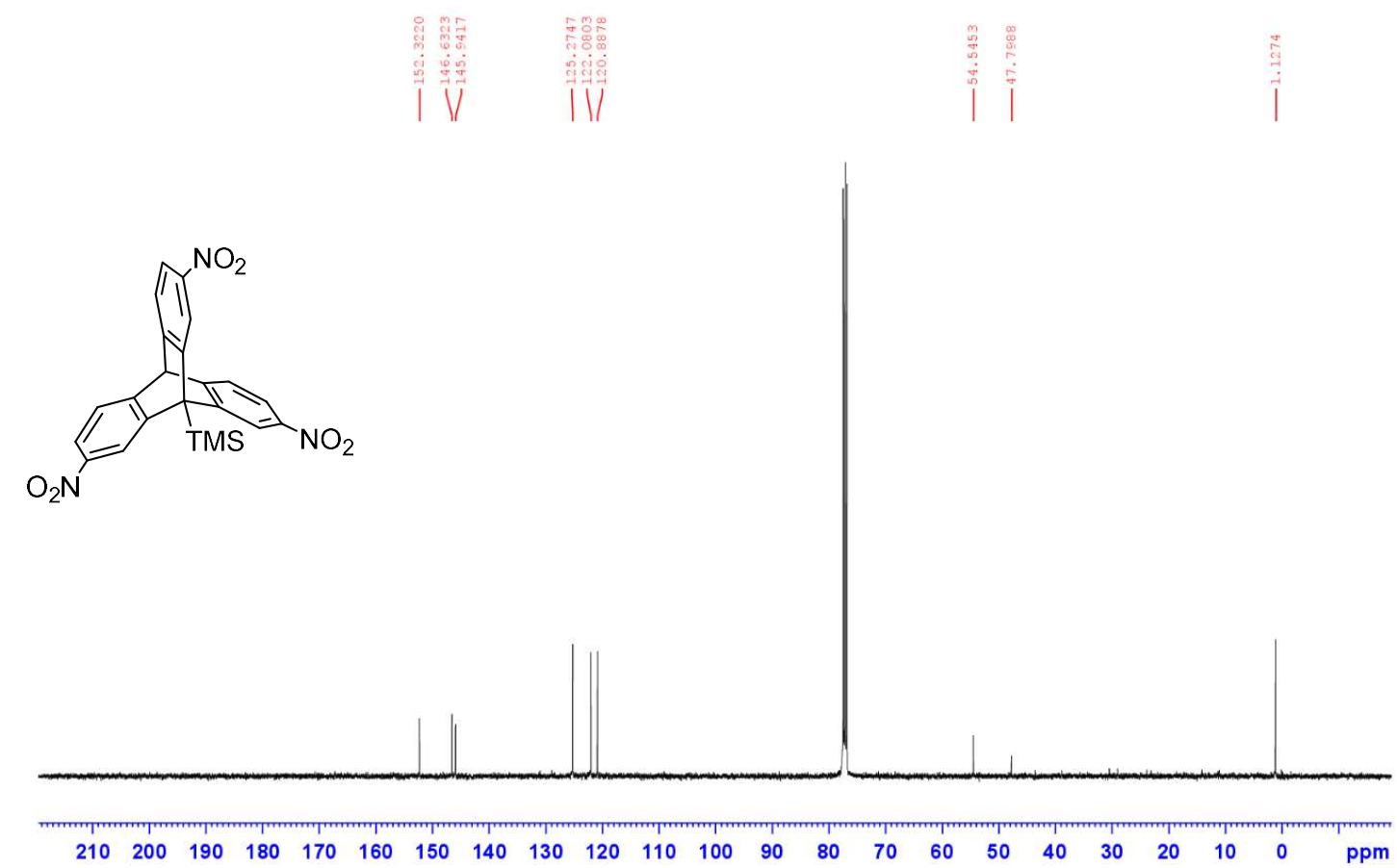


HSQC of $11\left(\mathrm{CDCl}_{3}\right)$

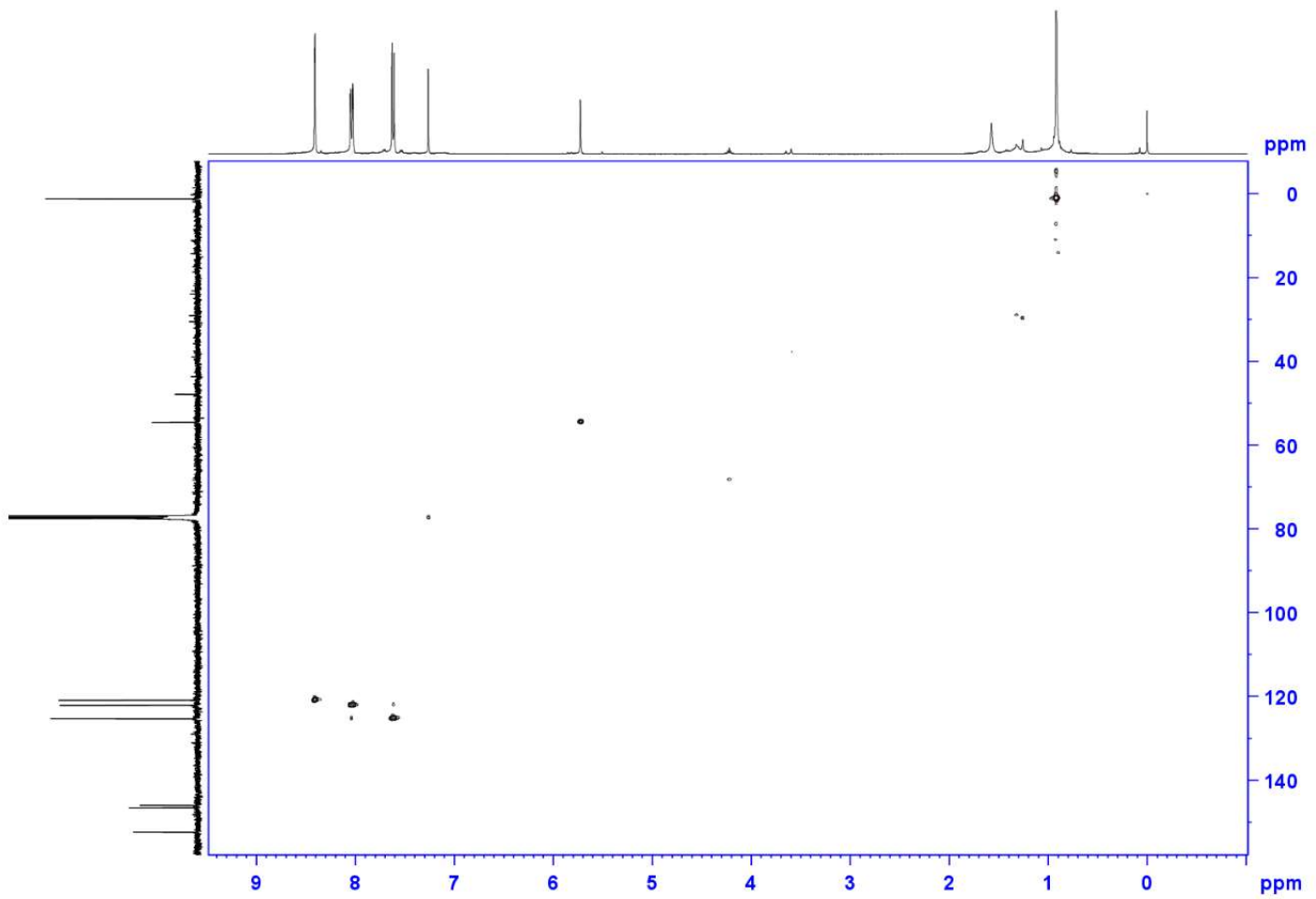

$\mathrm{HMBC}$ of $11\left(\mathrm{CDCl}_{3}\right)$

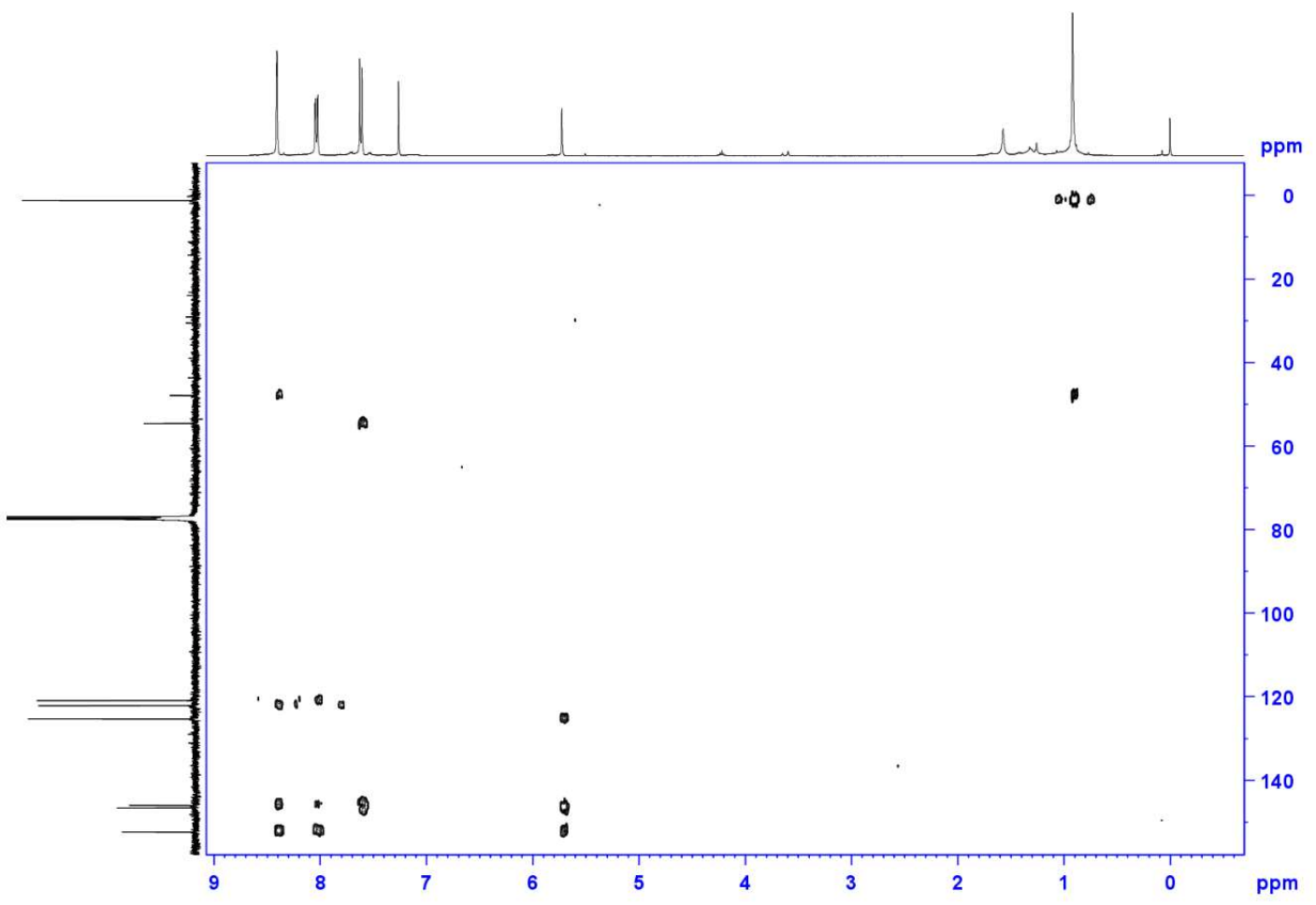


${ }^{1} \mathrm{H}$ NMR of isomer $\mathbf{B}\left(400 \mathrm{MHz}, \mathrm{CDCl}_{3}\right)$

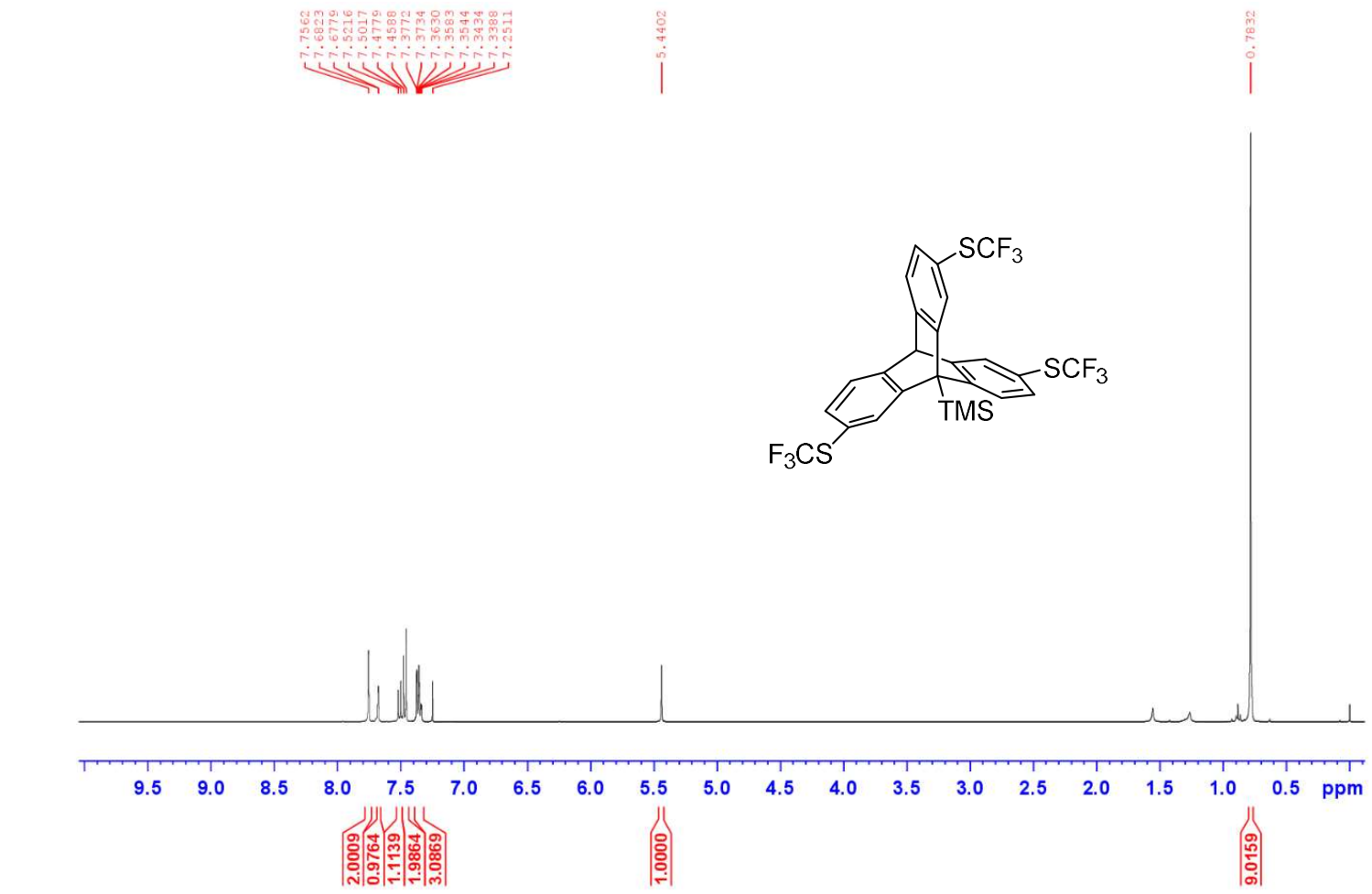

${ }^{13} \mathrm{C}$ NMR of isomer $\mathbf{B}\left(100 \mathrm{MHz}, \mathrm{CDCl}_{3}\right)$
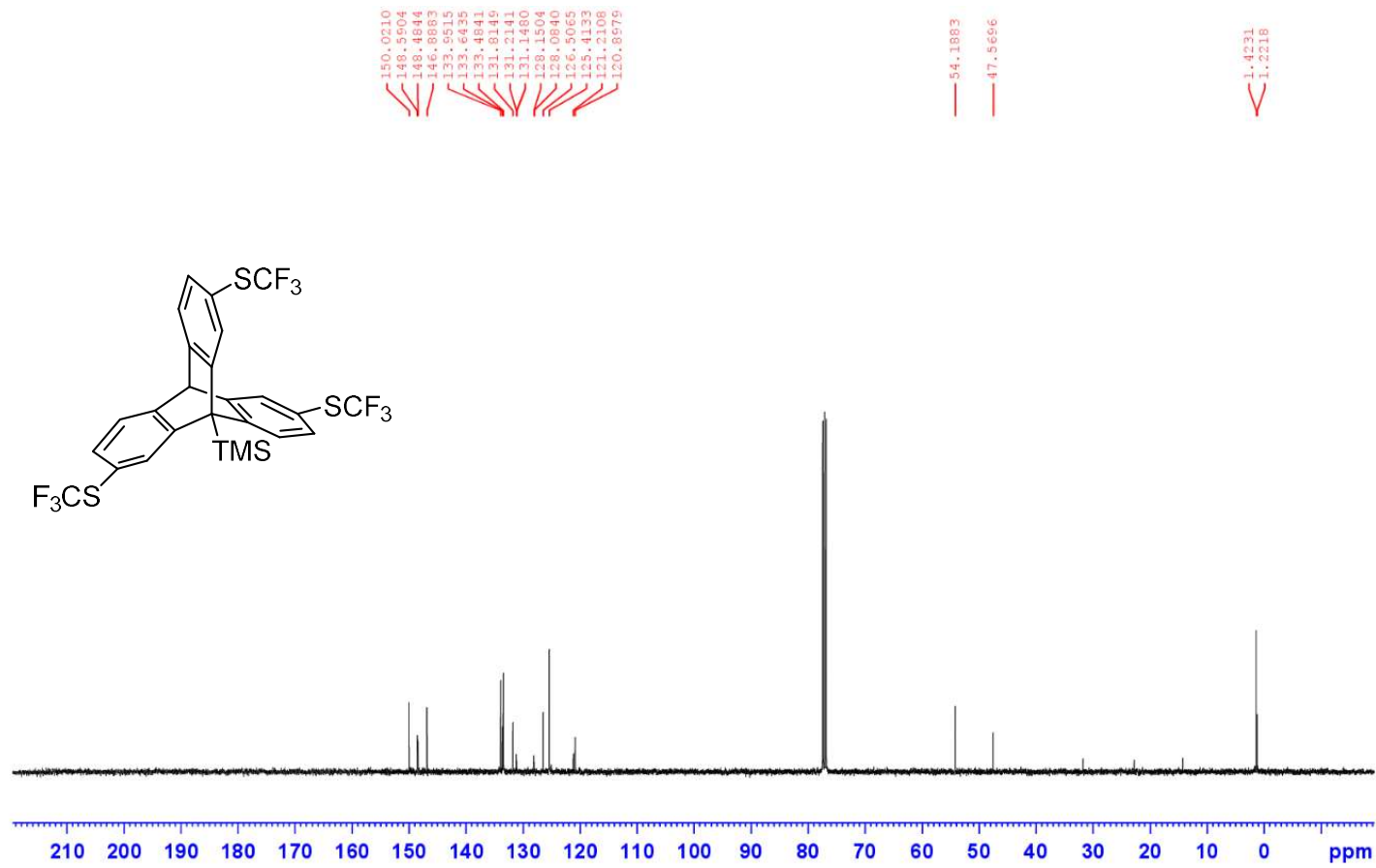
${ }^{19} \mathrm{~F} \mathrm{NMR}$ of isomer $\mathbf{B}\left(376 \mathrm{MHz}, \mathrm{CDCl}_{3}\right)$

V
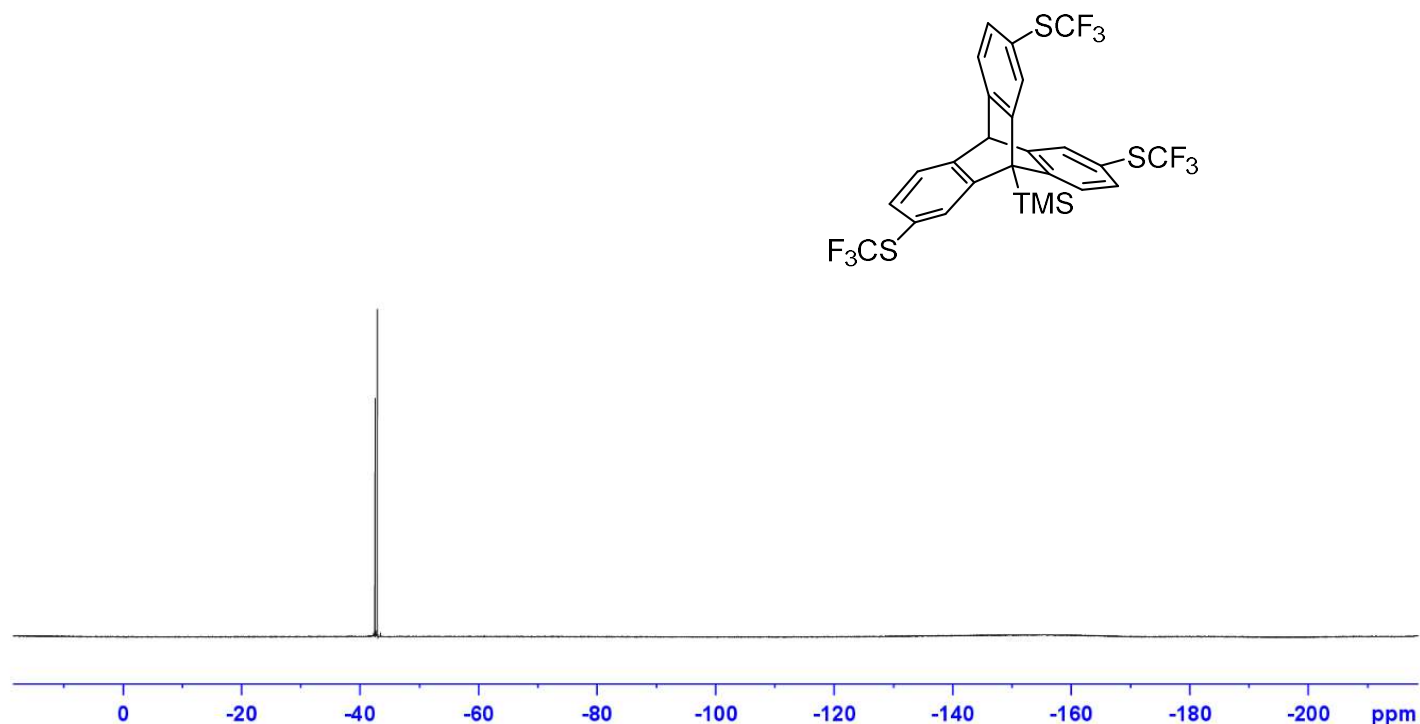

HSQC of isomer $\mathbf{B}\left(\mathrm{CDCl}_{3}\right)$

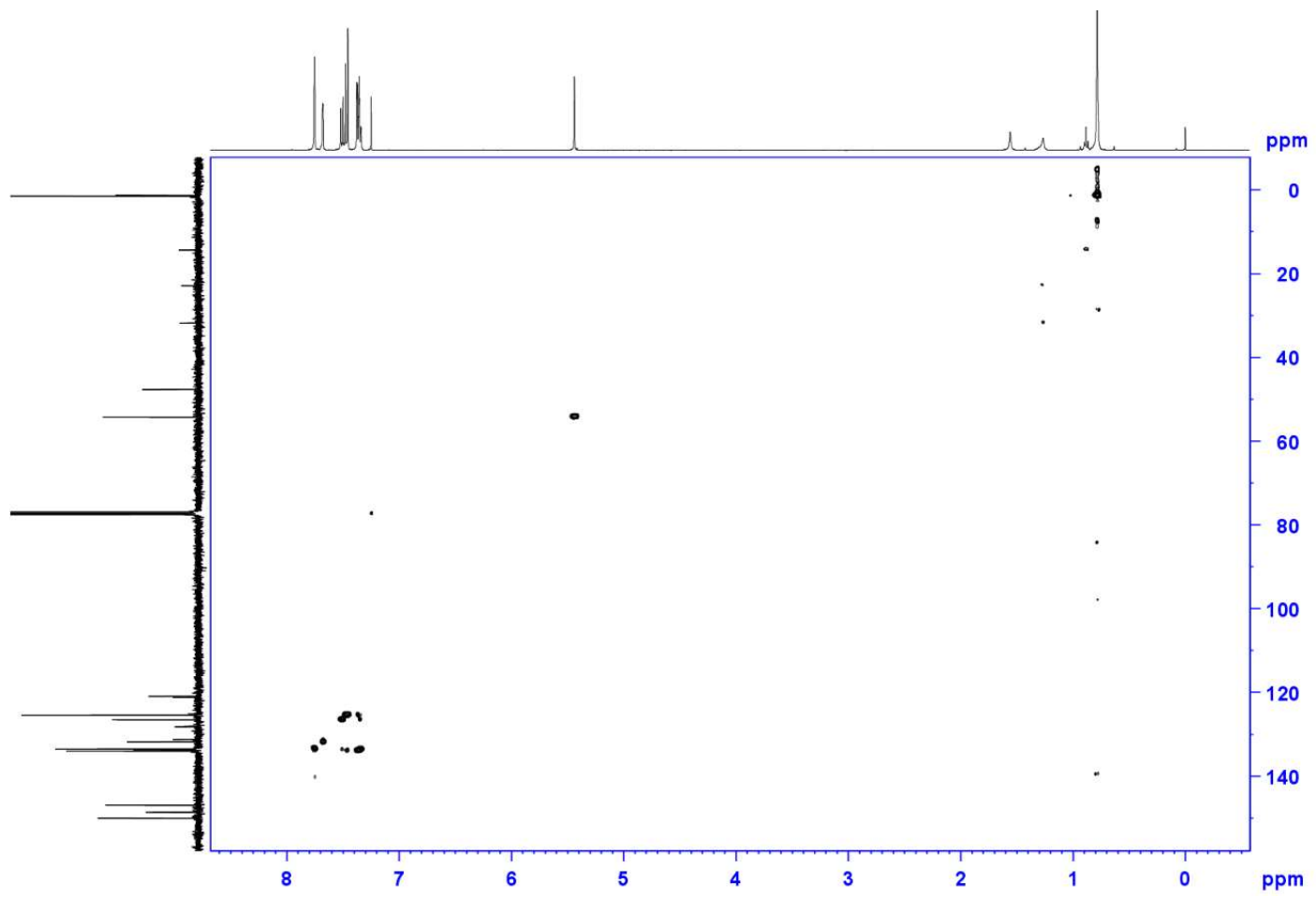


$\mathrm{HMBC}$ of isomer $\mathbf{B}\left(\mathrm{CDCl}_{3}\right)$

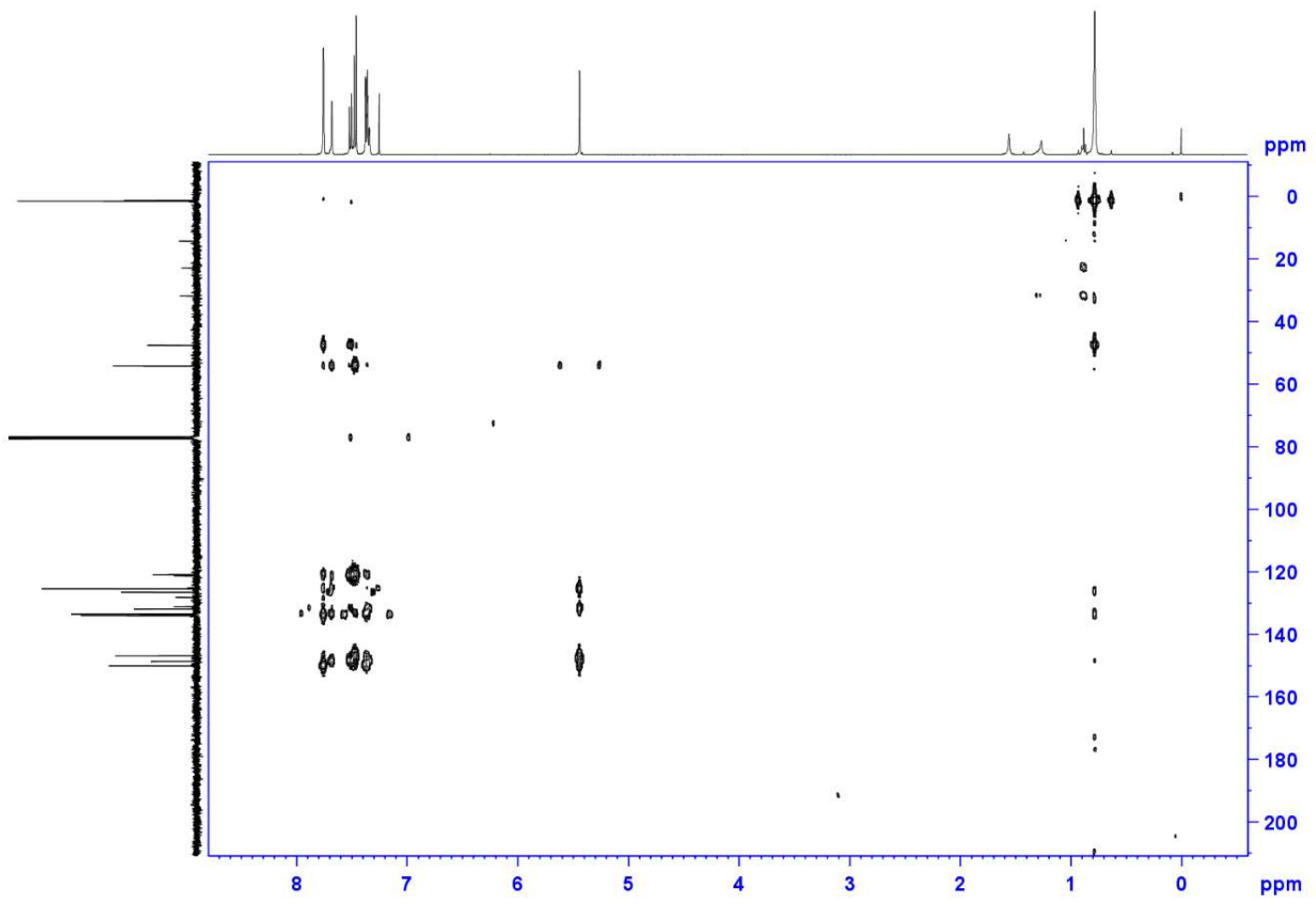


${ }^{1} \mathrm{H}$ NMR of isomer $\mathbf{C}\left(400 \mathrm{MHz}, \mathrm{CDCl}_{3}\right)$
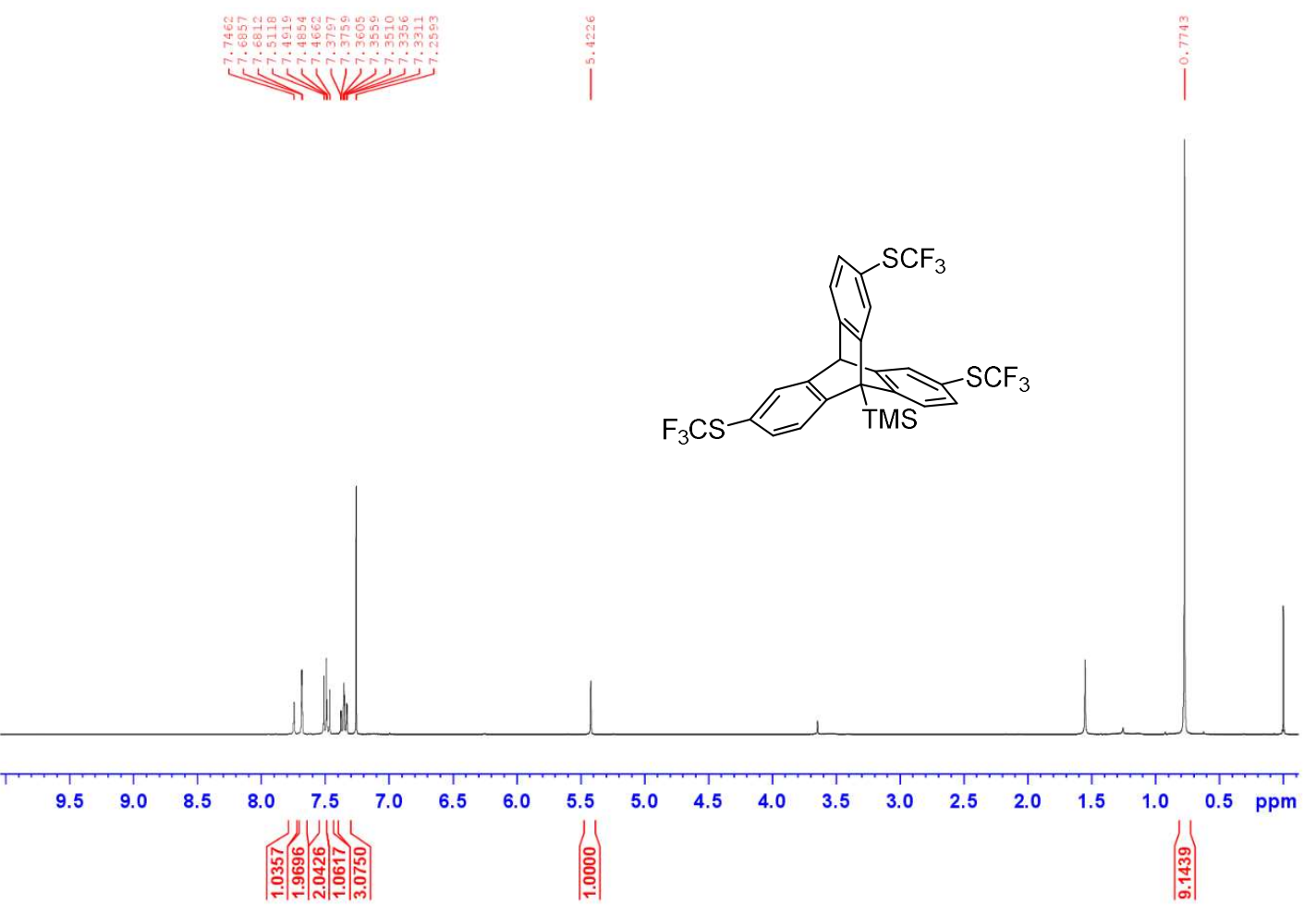

${ }^{13} \mathrm{C}$ NMR of isomer $\mathbf{C}\left(100 \mathrm{MHz}, \mathrm{CDCl}_{3}\right)$

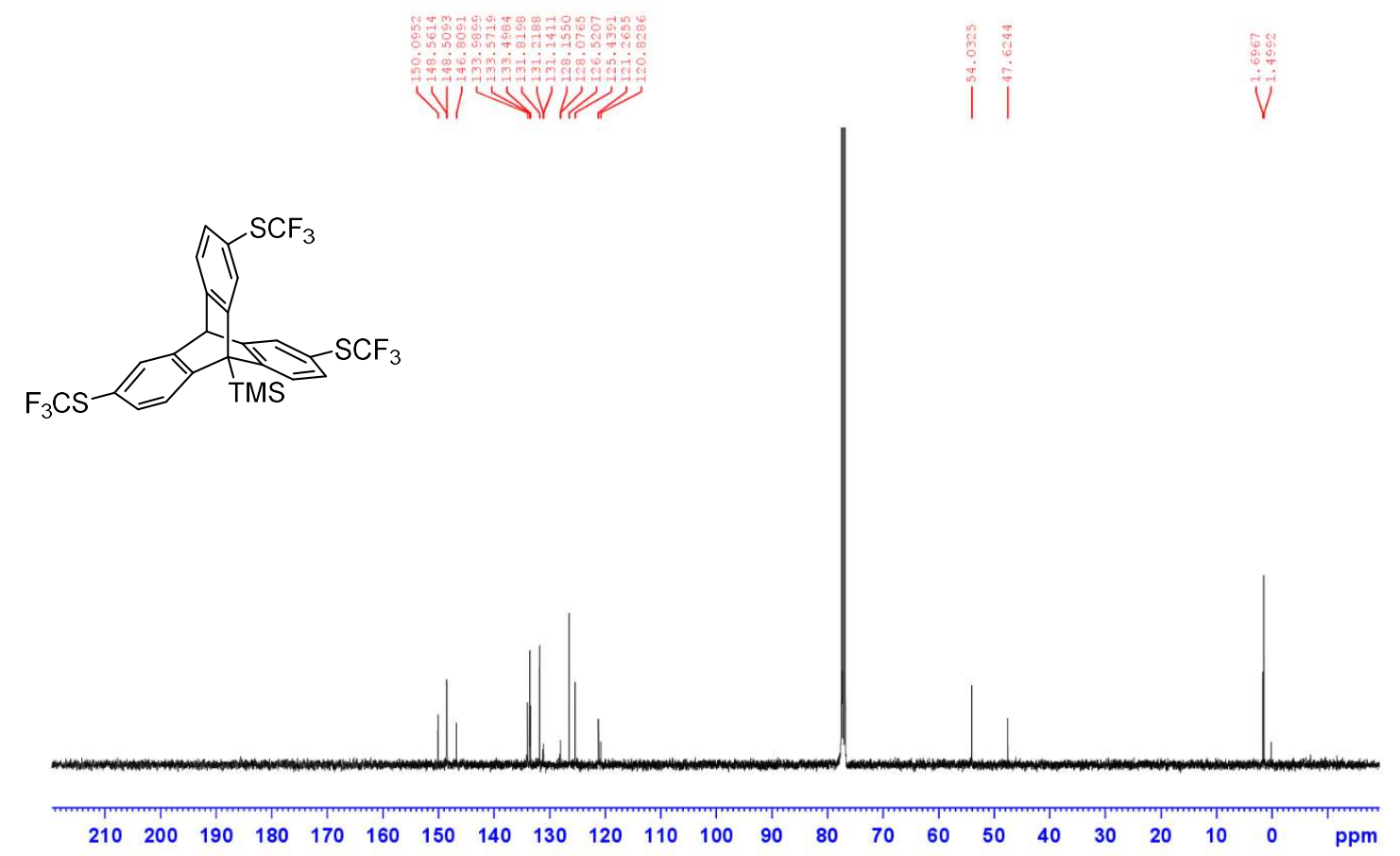


${ }^{19} \mathrm{~F} \mathrm{NMR}$ of isomer $\mathbf{C}\left(376 \mathrm{MHz}, \mathrm{CDCl}_{3}\right)$

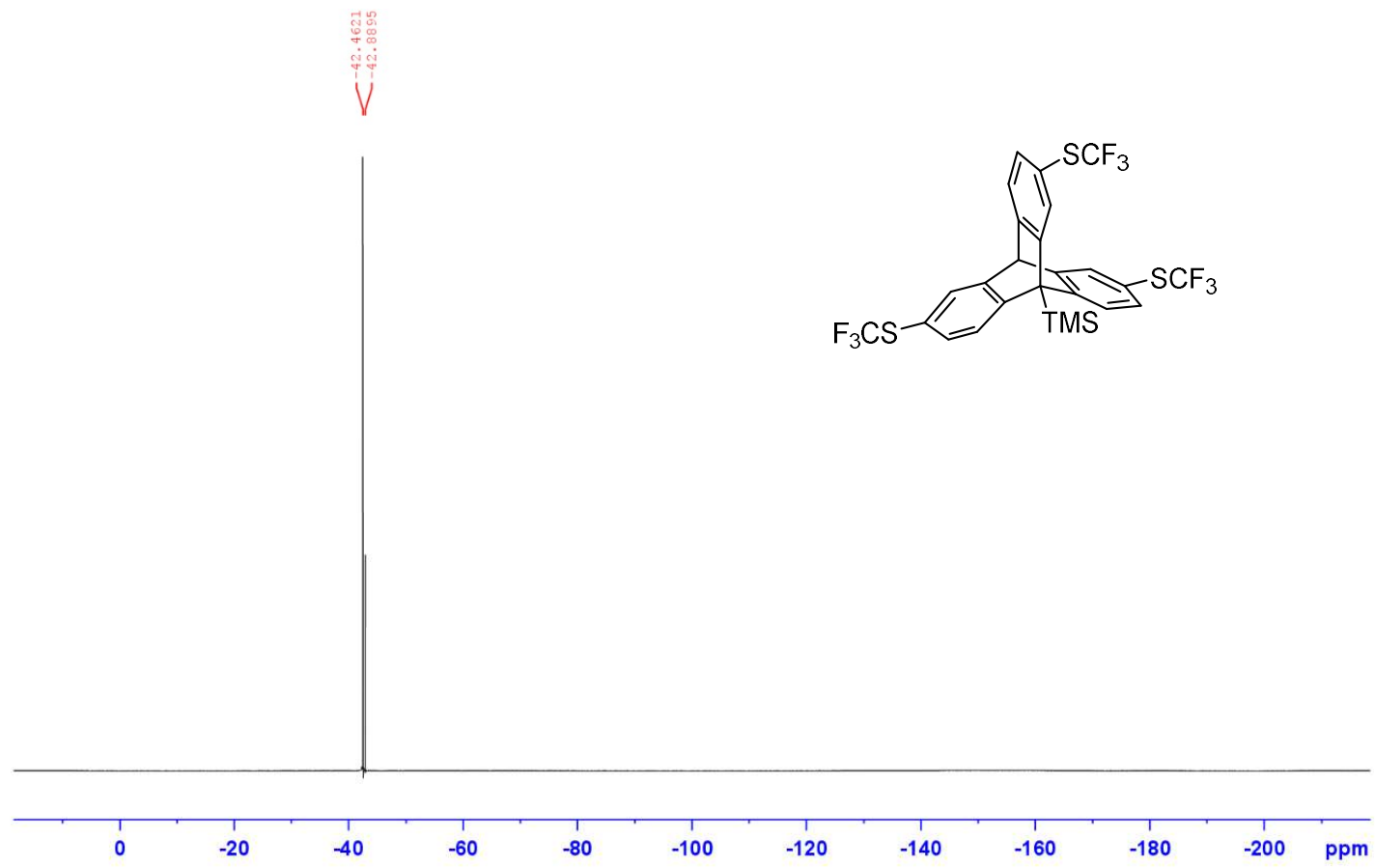

HSQC of isomer $\mathbf{C}\left(\mathrm{CDCl}_{3}\right)$

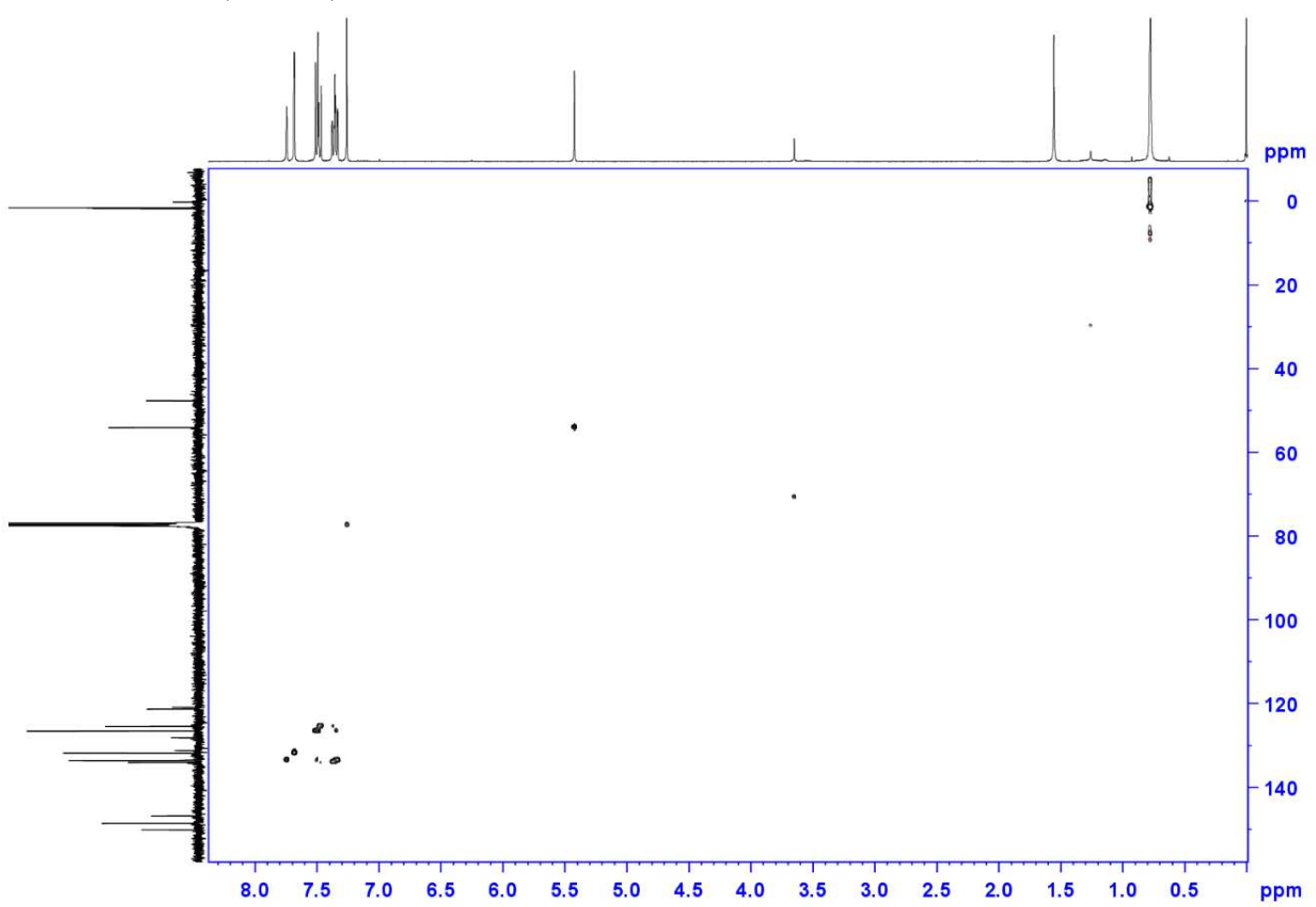


HMBC of isomer $\mathbf{C}\left(\mathrm{CDCl}_{3}\right)$

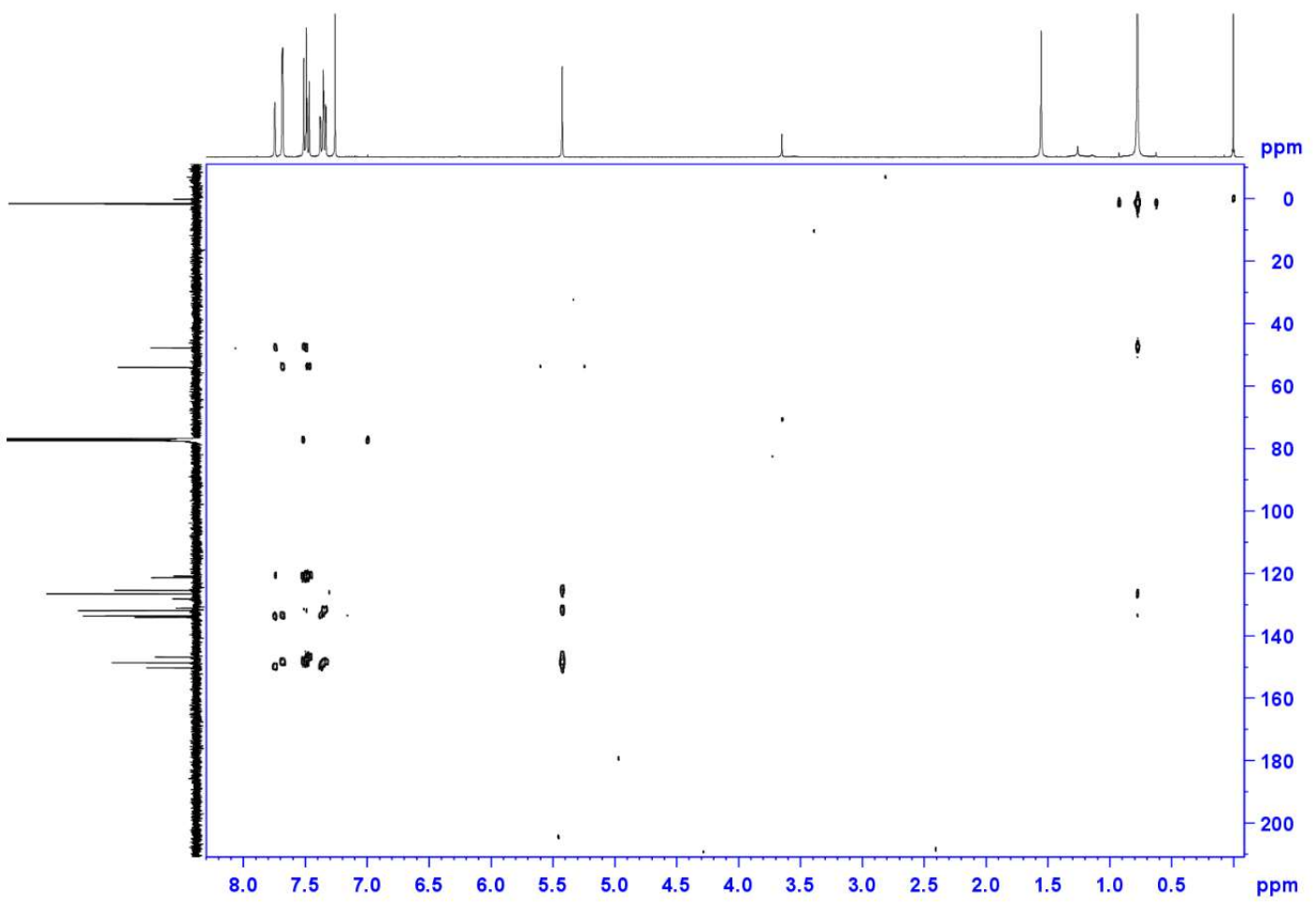


${ }^{1} \mathrm{H}$ NMR of isomer $\mathbf{D}\left(400 \mathrm{MHz}, \mathrm{CDCl}_{3}\right)$

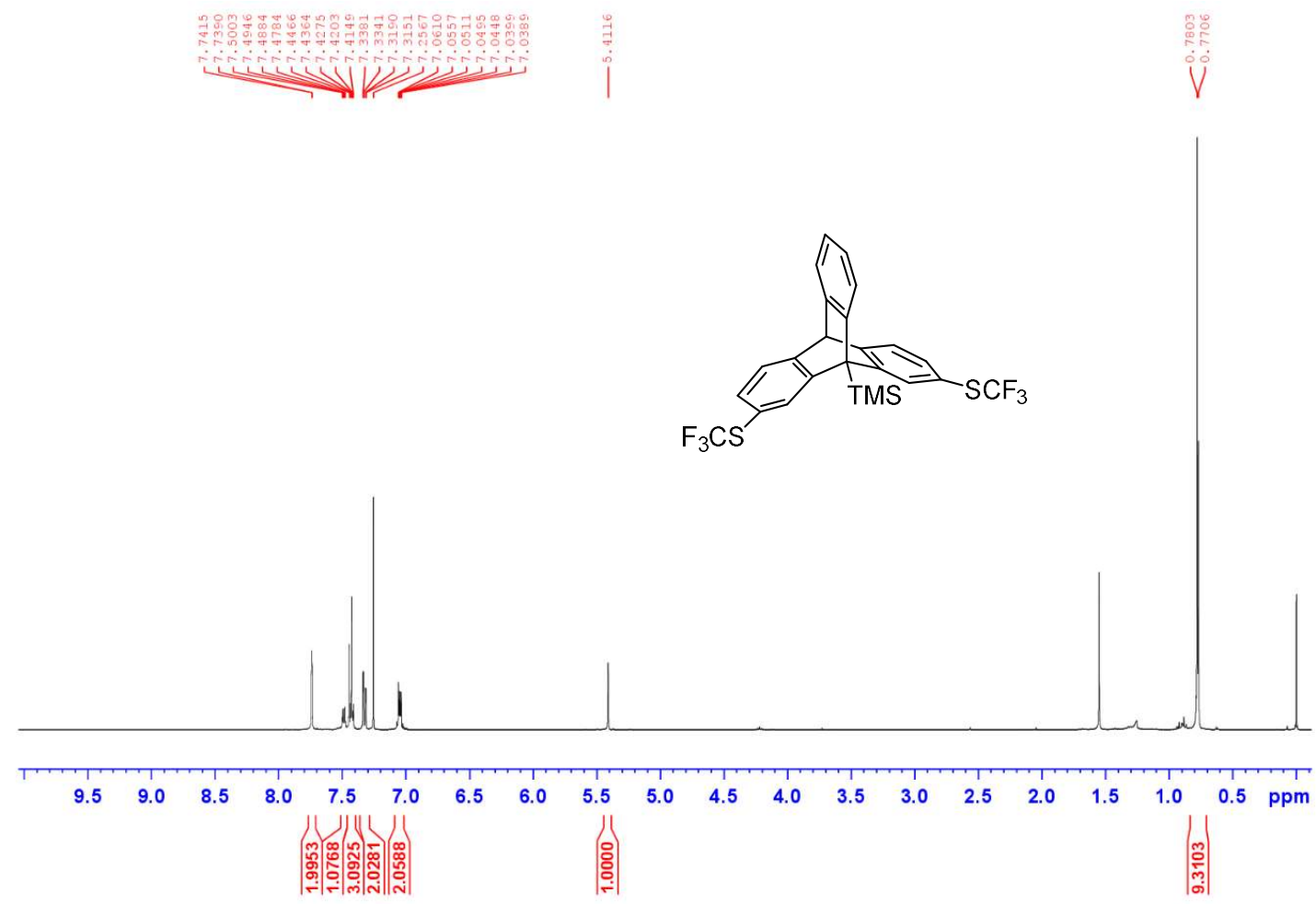

${ }^{13} \mathrm{C}$ NMR of isomer $\mathbf{D}\left(100 \mathrm{MHz}, \mathrm{CDCl}_{3}\right)$

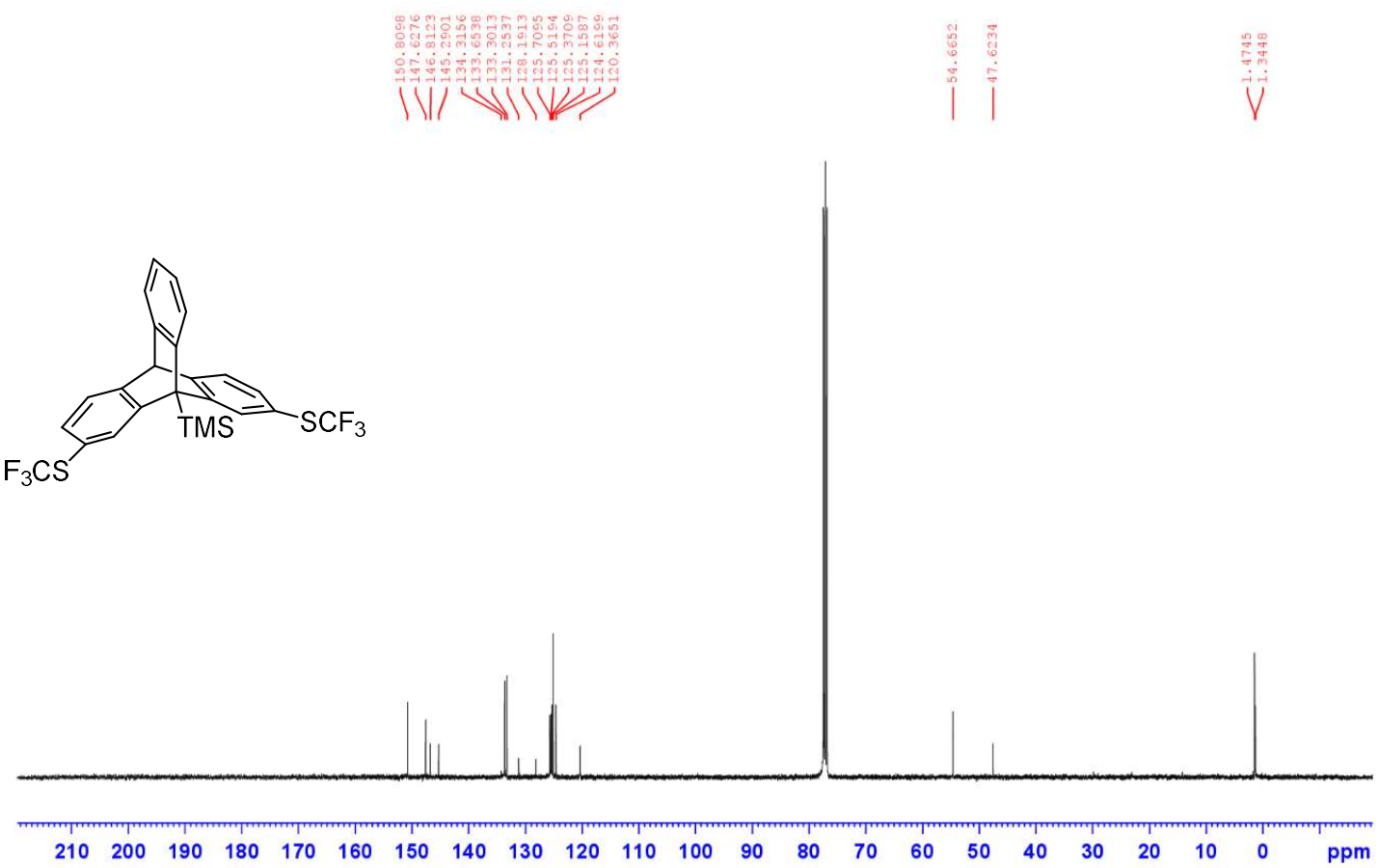


${ }^{19} \mathrm{~F}$ NMR of isomer $\mathbf{D}\left(376 \mathrm{MHz}, \mathrm{CDCl}_{3}\right)$

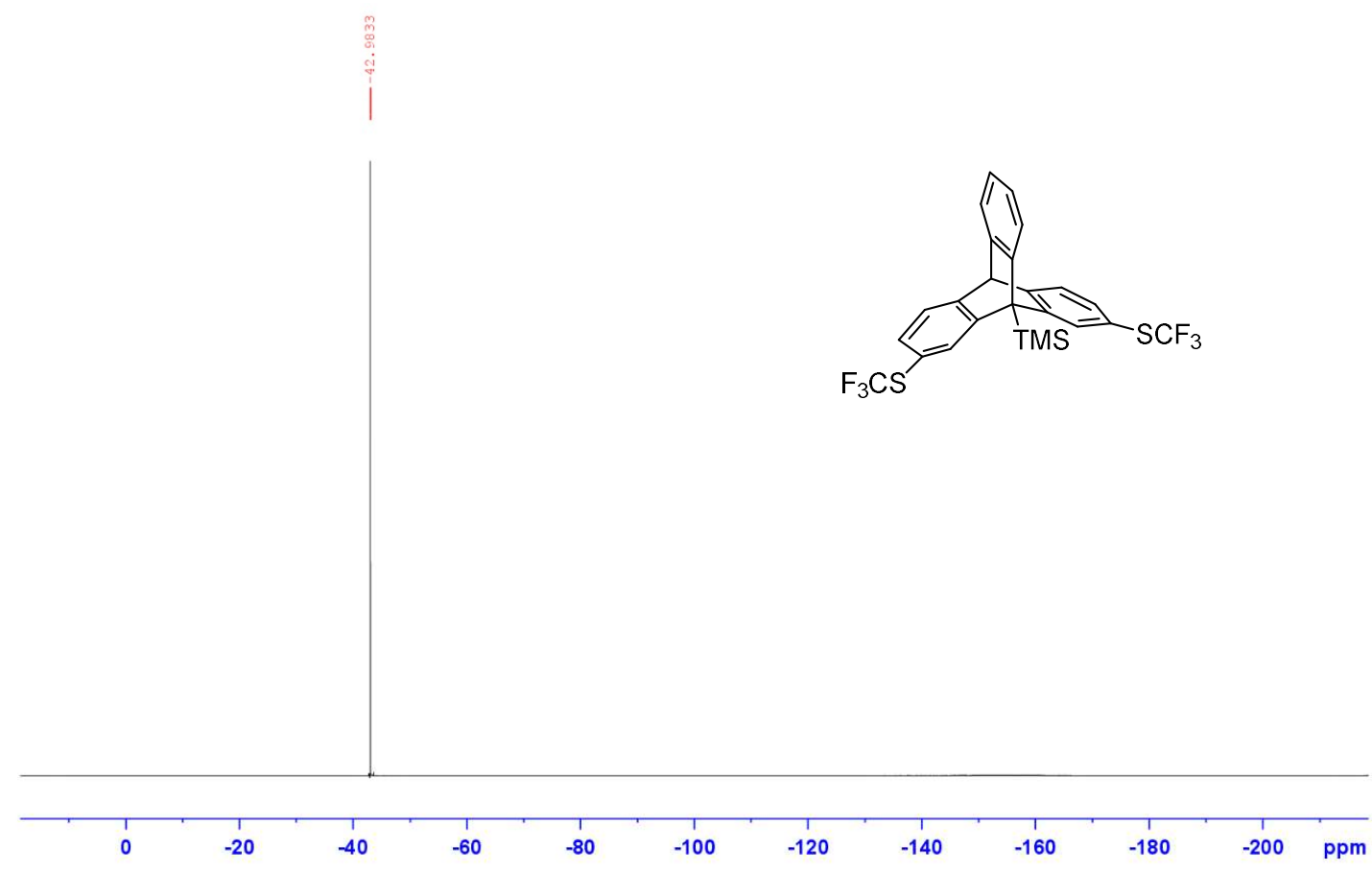

HSQC of isomer $\mathbf{D}\left(\mathrm{CDCl}_{3}\right)$

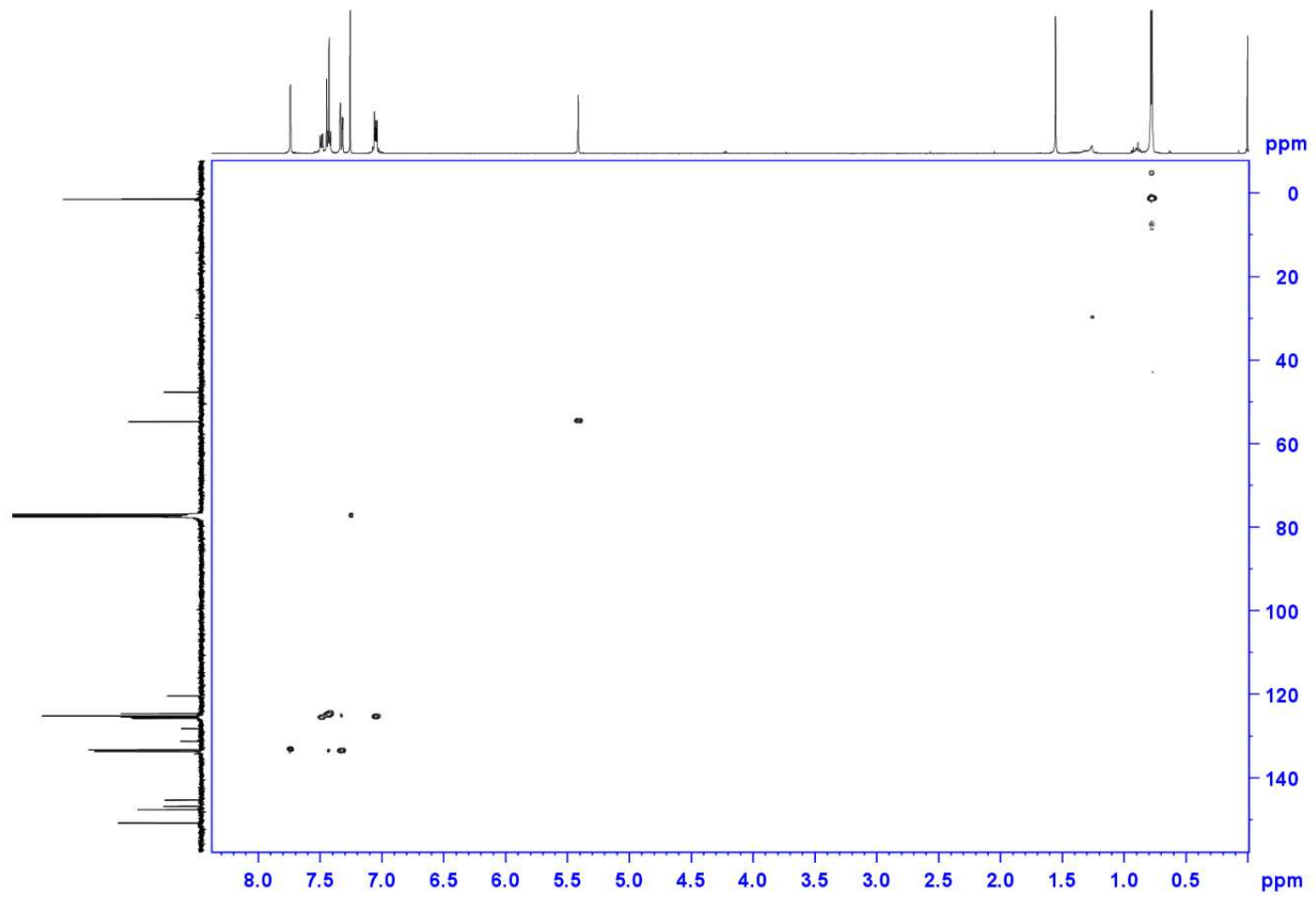


HMBC of isomer $\mathbf{D}\left(\mathrm{CDCl}_{3}\right)$

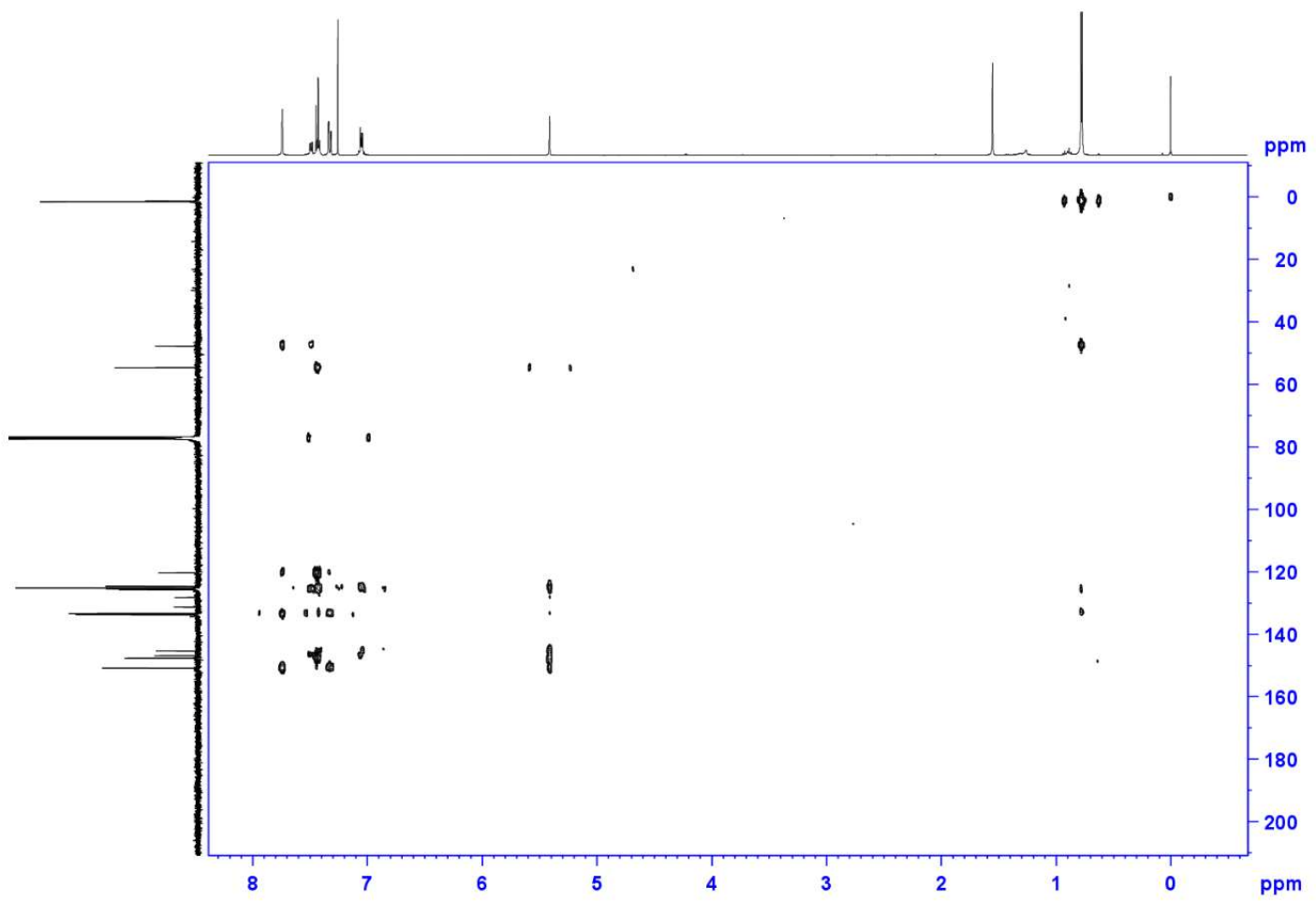


${ }^{1} \mathrm{H}$ NMR of isomer $\mathbf{G}\left(400 \mathrm{MHz}, \mathrm{CDCl}_{3}\right)$

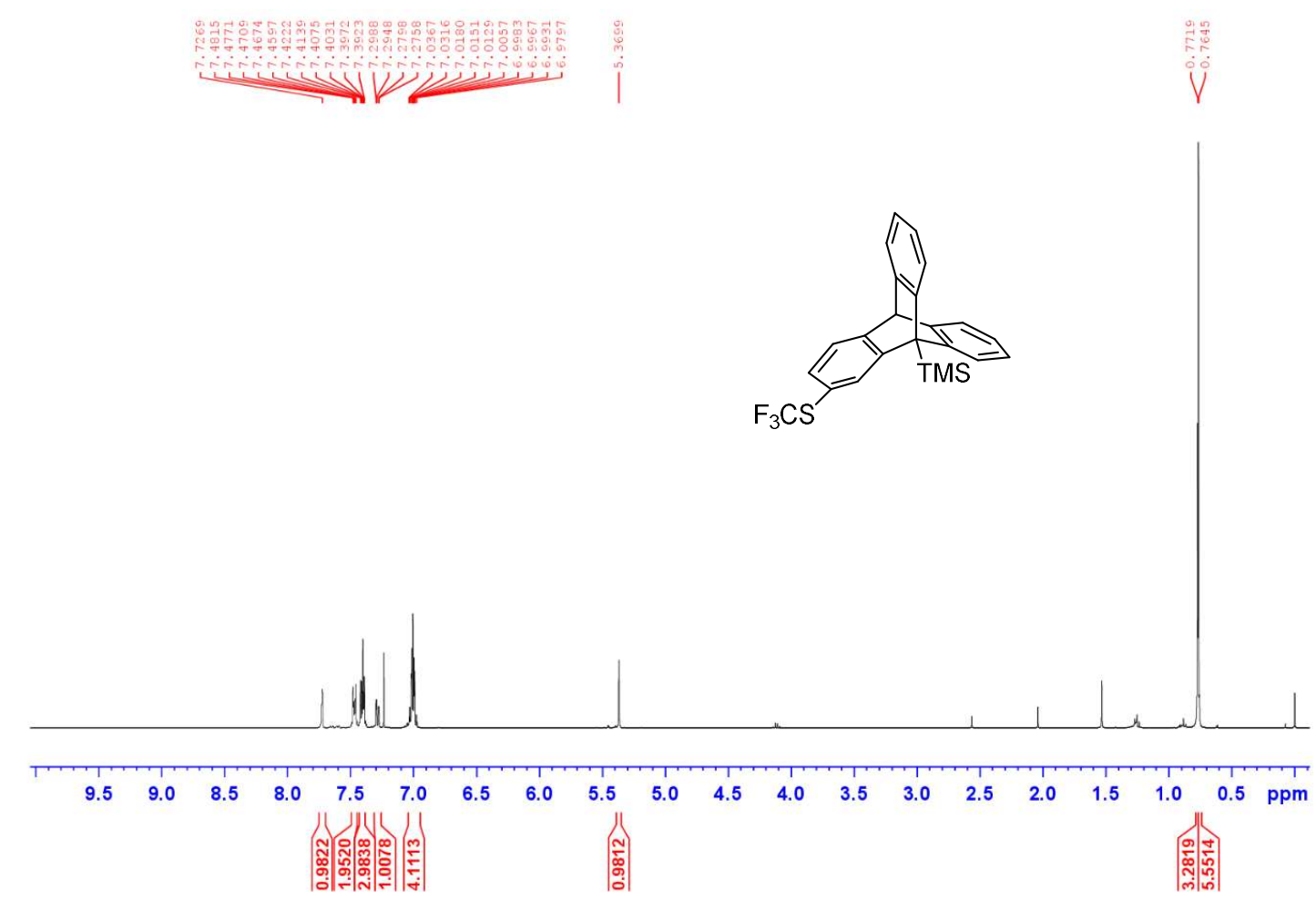

${ }^{13} \mathrm{C}$ NMR of isomer $\mathbf{G}\left(100 \mathrm{MHz}, \mathrm{CDCl}_{3}\right)$
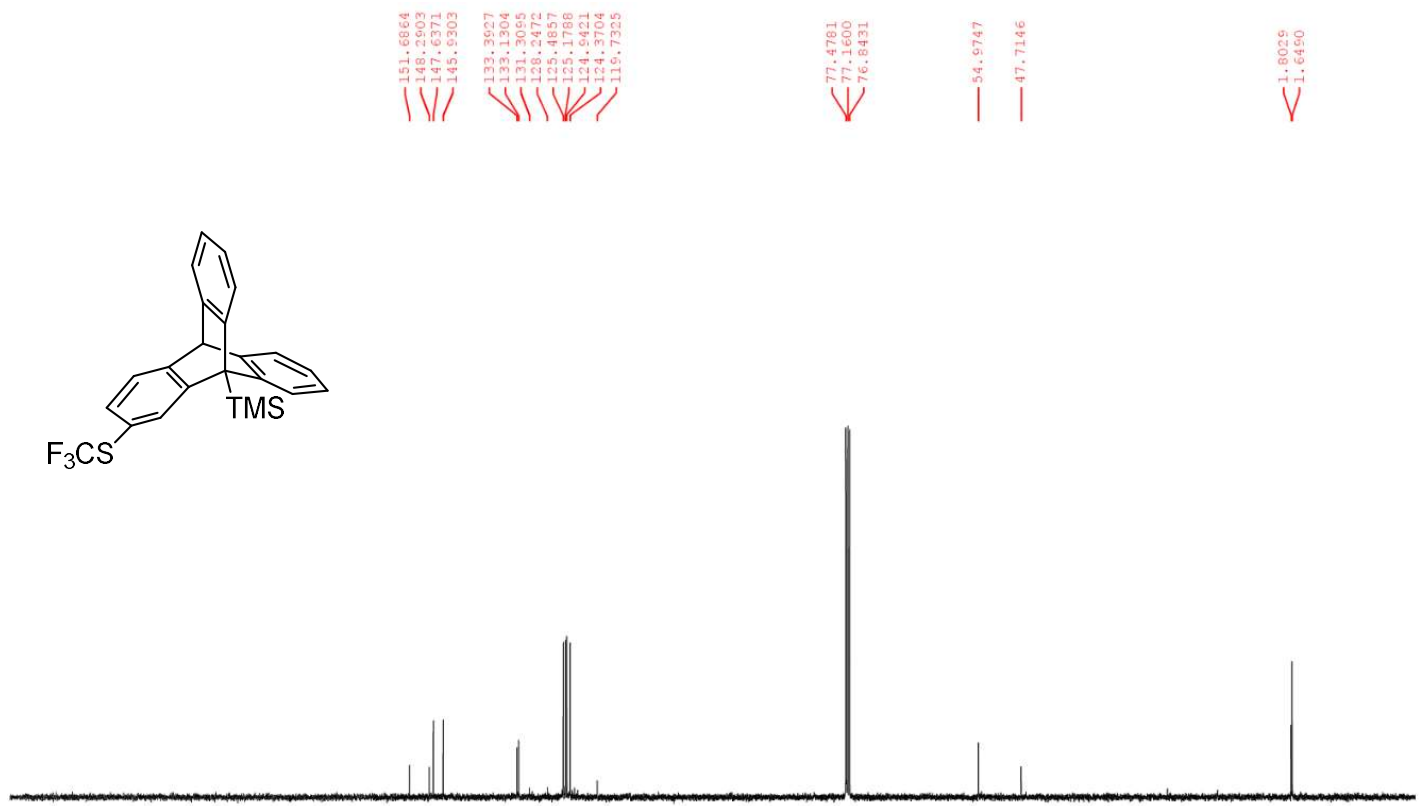

$\begin{array}{llllllllllllllllllllll}210 & 200 & 190 & 180 & 170 & 160 & 150 & 140 & 130 & 120 & 110 & 100 & 90 & 80 & 70 & 60 & 50 & 40 & 30 & 20 & 10 & 0\end{array}$ 
${ }^{19} \mathrm{~F}$ NMR of isomer $\mathbf{G}\left(376 \mathrm{MHz}, \mathrm{CDCl}_{3}\right)$

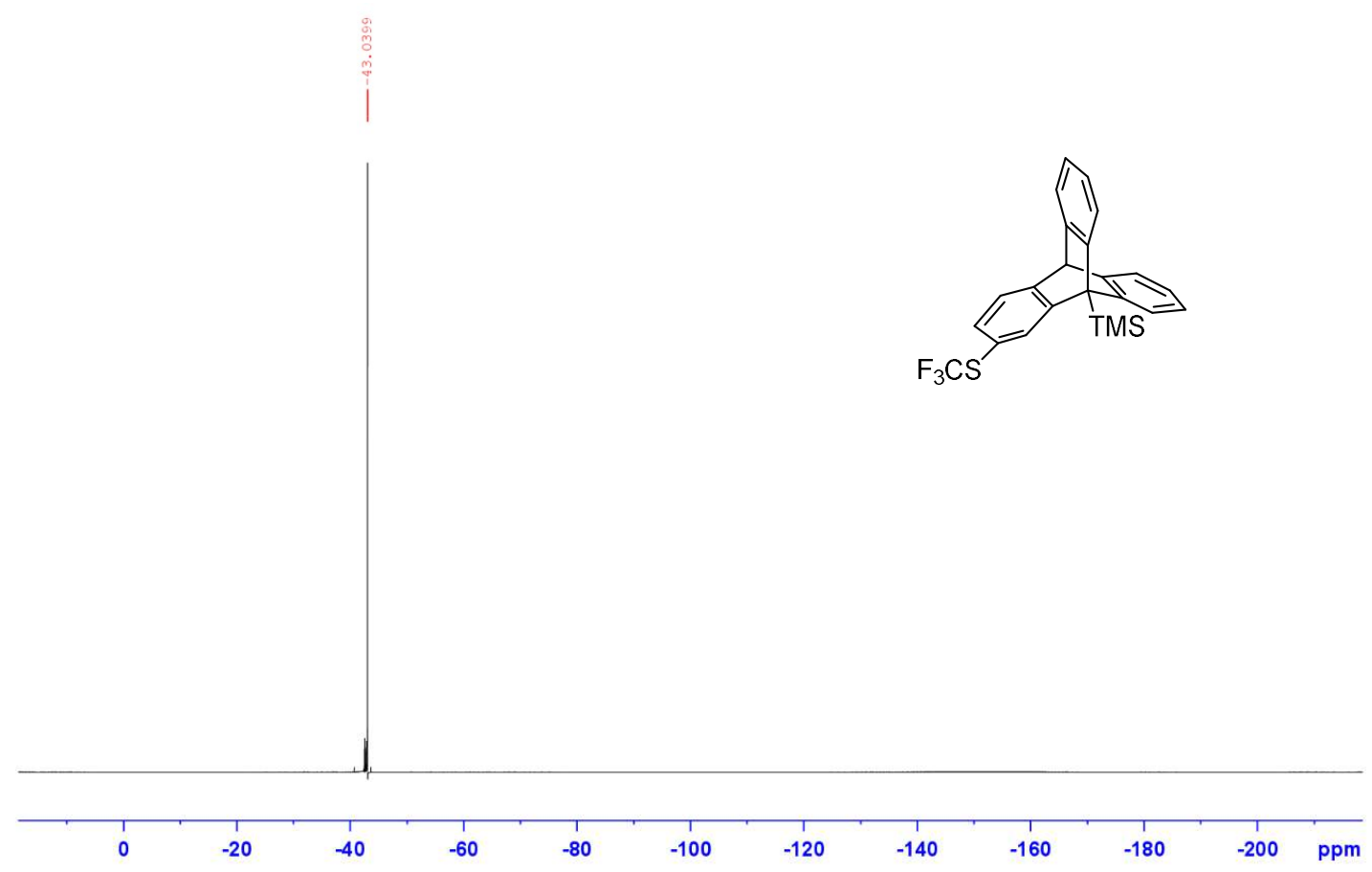

HSQC of isomer $\mathbf{G}\left(\mathrm{CDCl}_{3}\right)$

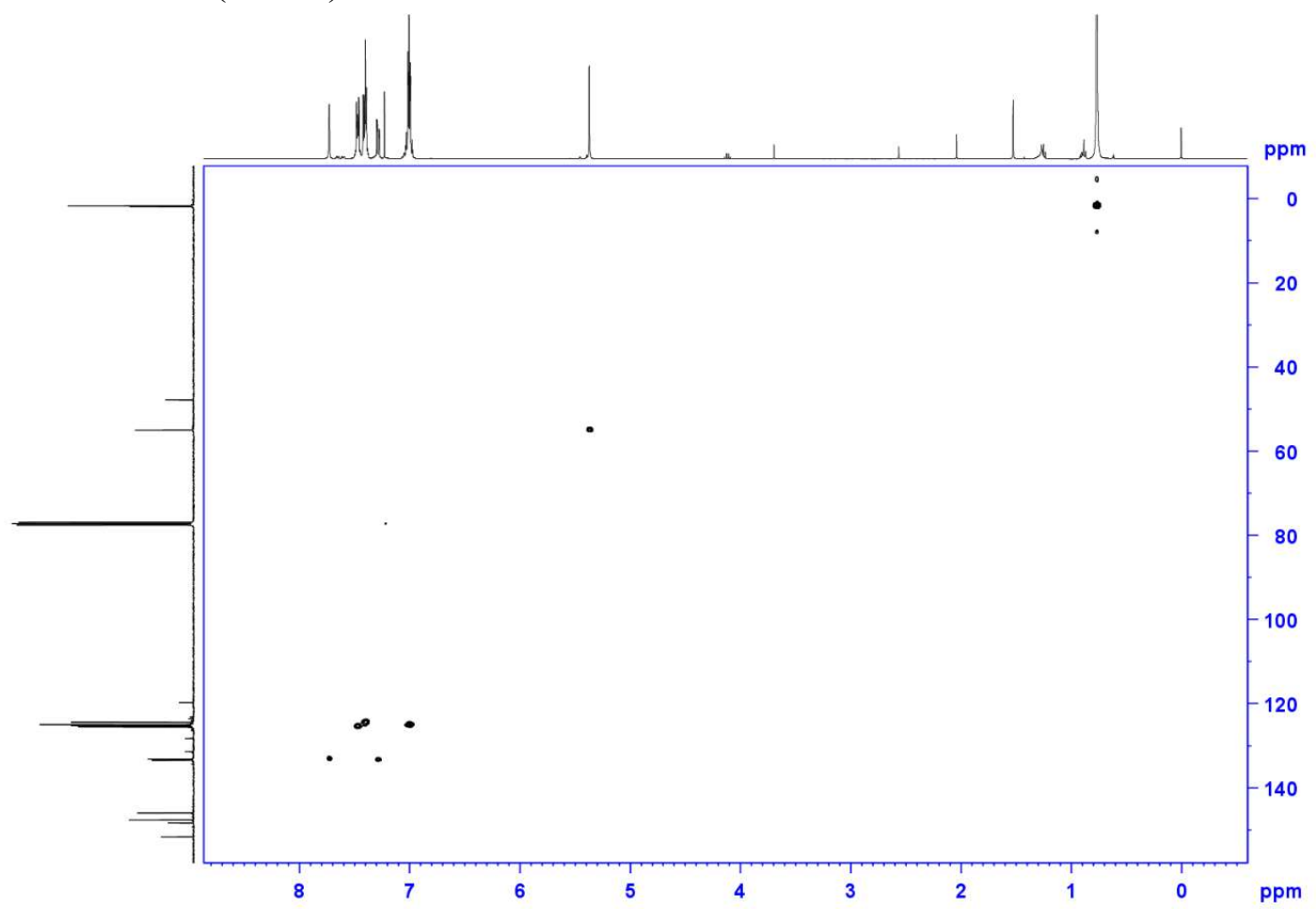


$\mathrm{HMBC}$ of isomer $\mathbf{G}\left(\mathrm{CDCl}_{3}\right)$

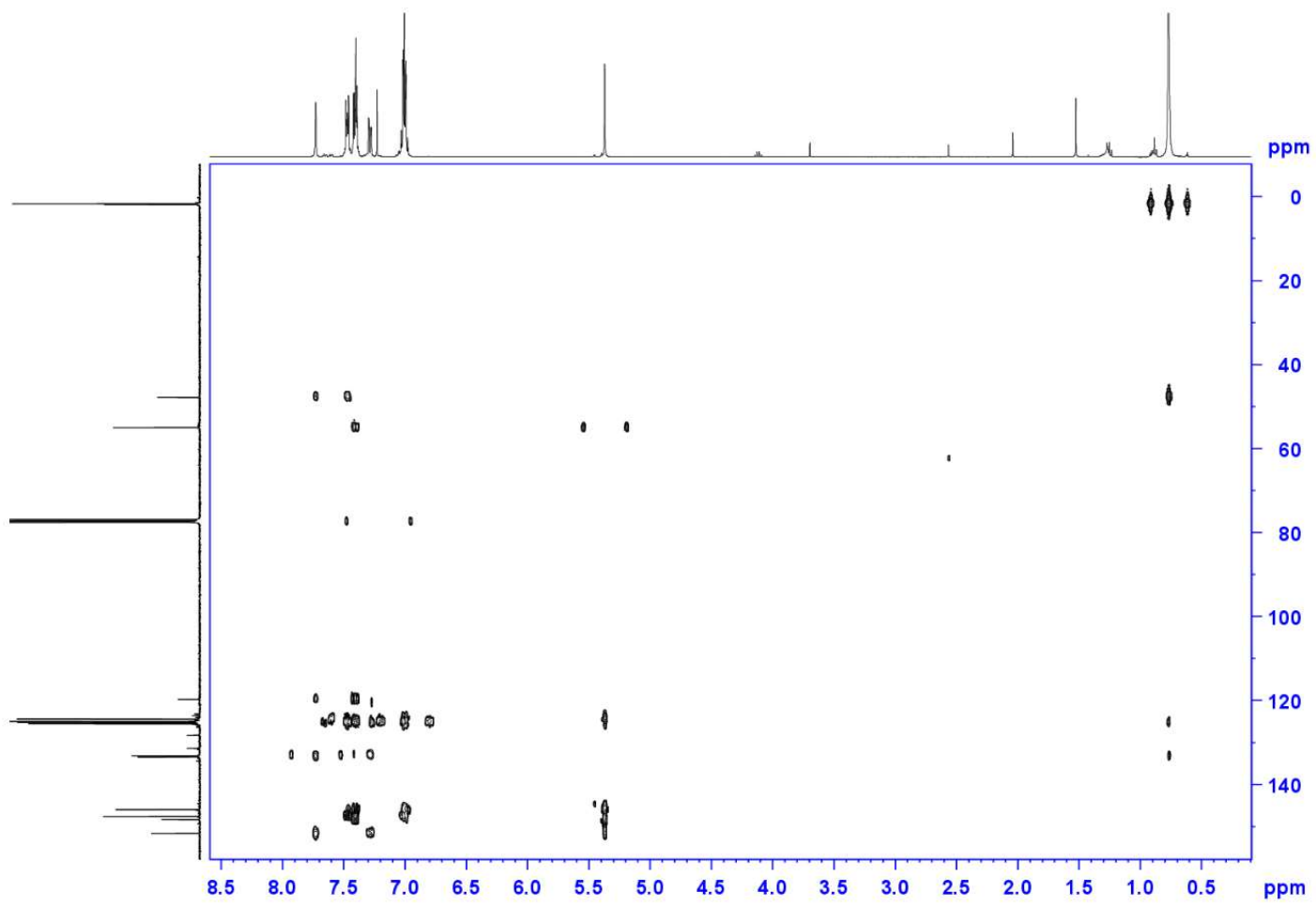


${ }^{1} \mathrm{H}$ NMR of isomer $\mathbf{J}\left(400 \mathrm{MHz}, \mathrm{CDCl}_{3}\right)$

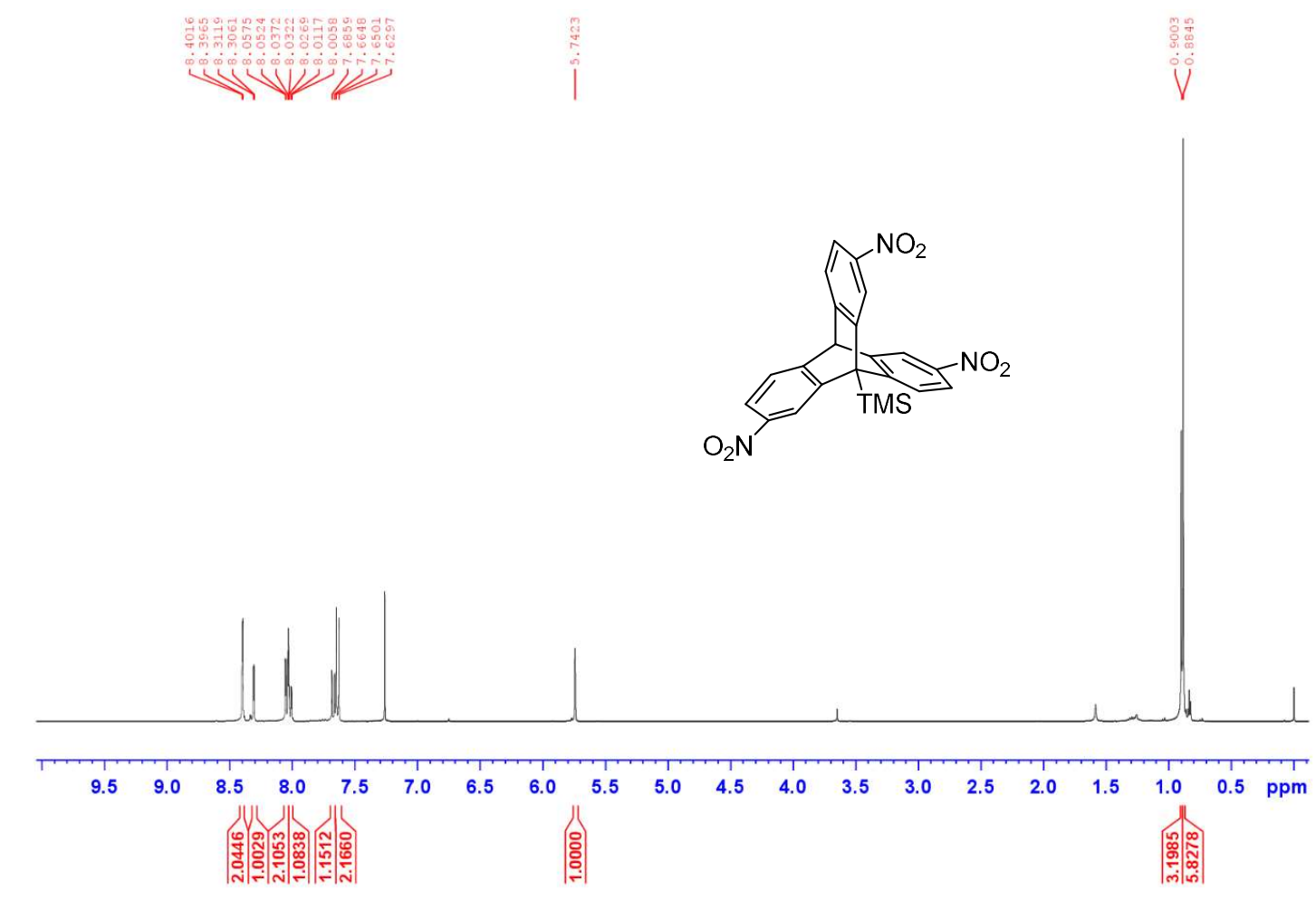

${ }^{13} \mathrm{C} \mathrm{NMR}$ of isomer $\mathbf{J}\left(100 \mathrm{MHz}, \mathrm{CDCl}_{3}\right)$

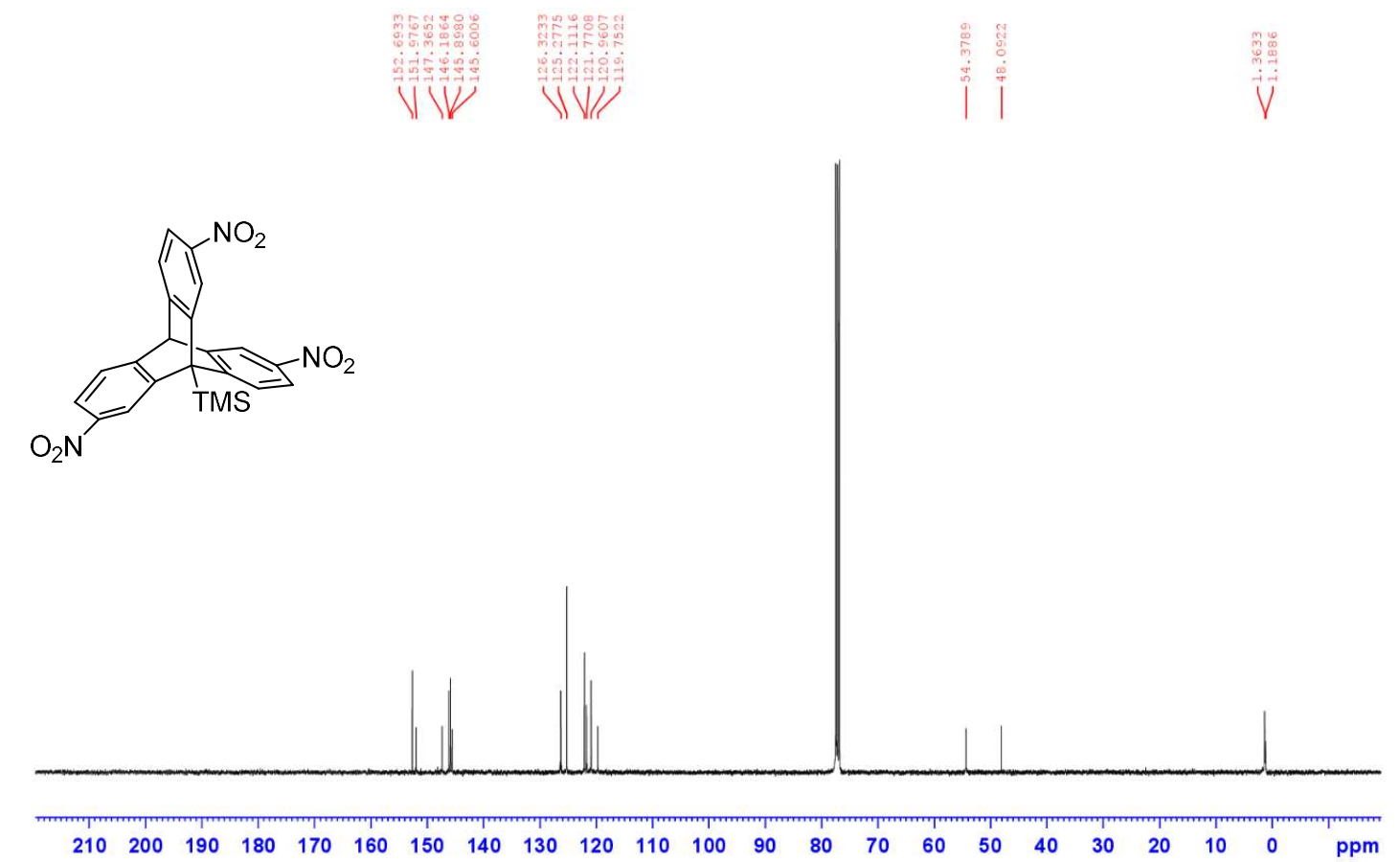


HSQC of isomer $\mathbf{J}\left(\mathrm{CDCl}_{3}\right)$

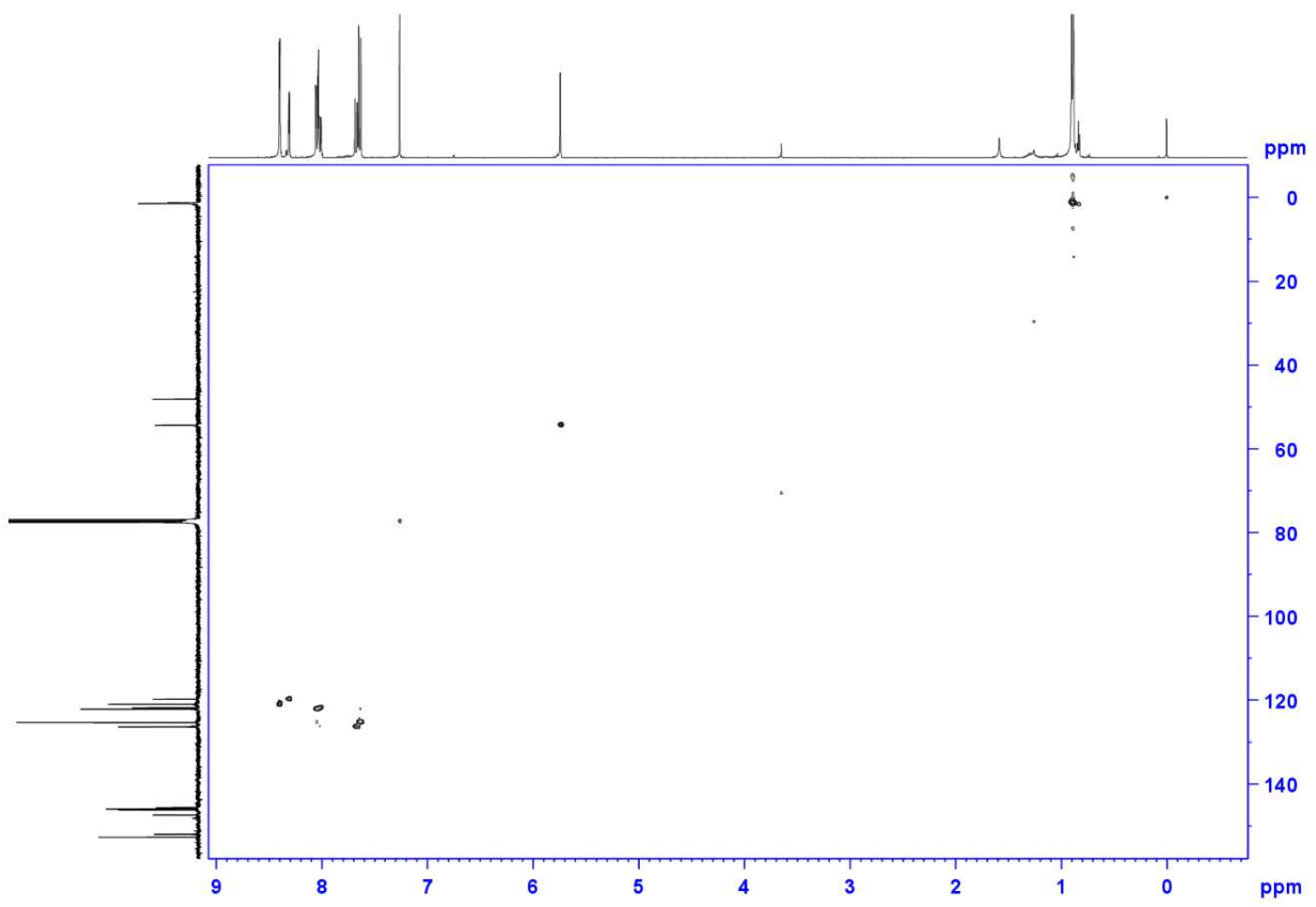

$\mathrm{HMBC}$ of isomer $\mathbf{J}\left(\mathrm{CDCl}_{3}\right)$

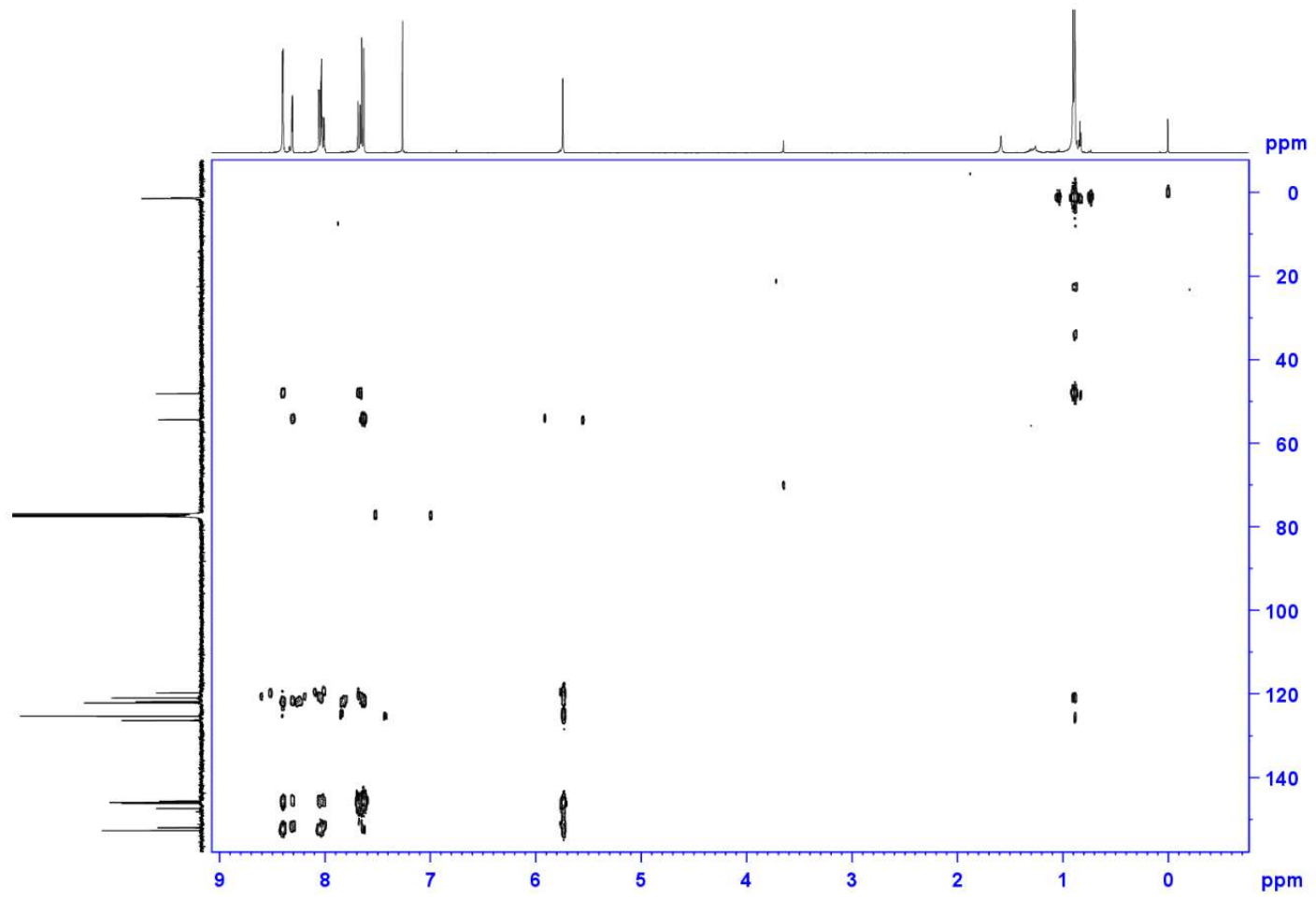


${ }^{1} \mathrm{H}$ NMR of isomer $\mathbf{K}\left(400 \mathrm{MHz}, \mathrm{CDCl}_{3}\right)$
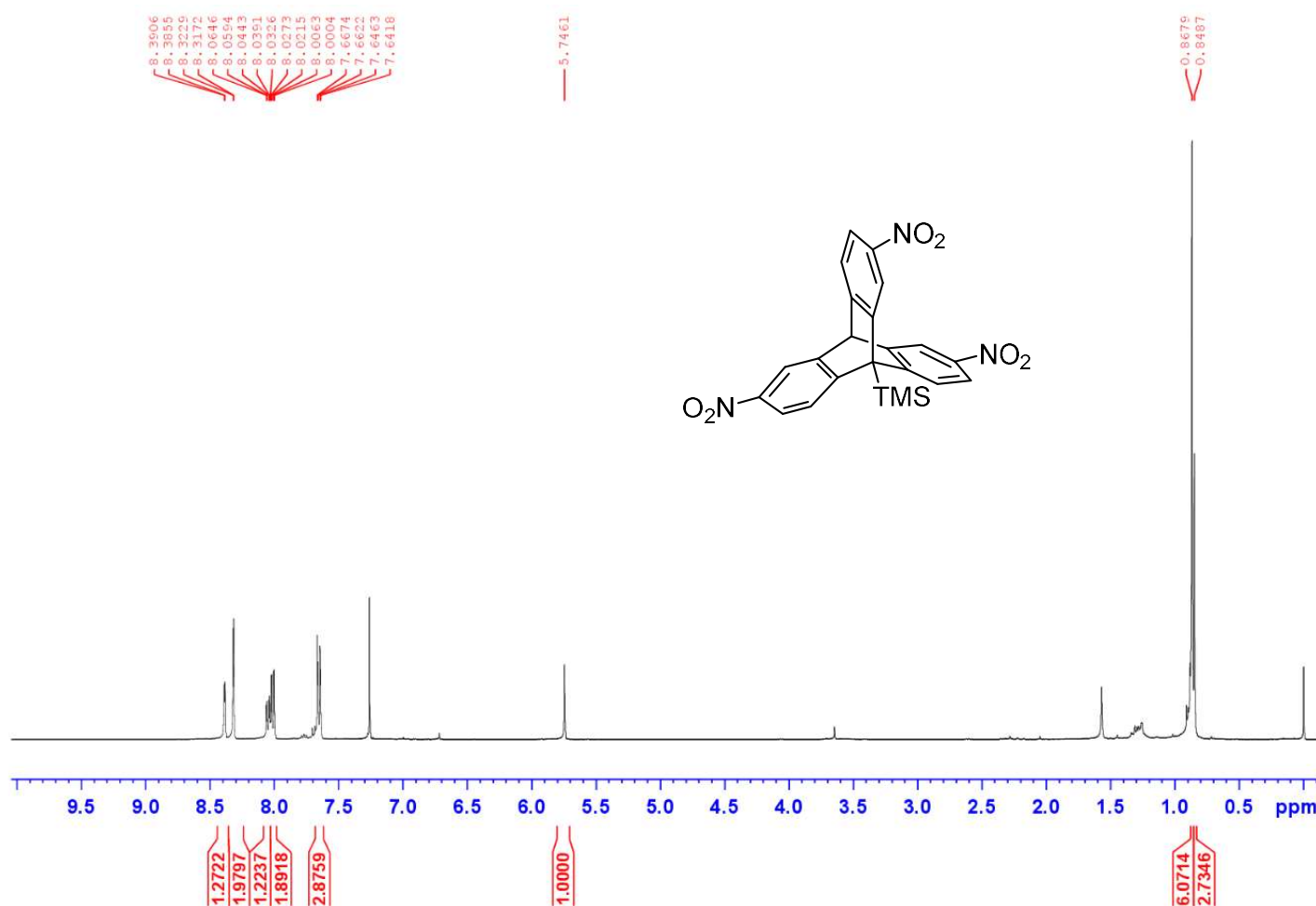

${ }^{13} \mathrm{C}$ NMR of isomer $\mathbf{K}\left(100 \mathrm{MHz}, \mathrm{CDCl}_{3}\right)$
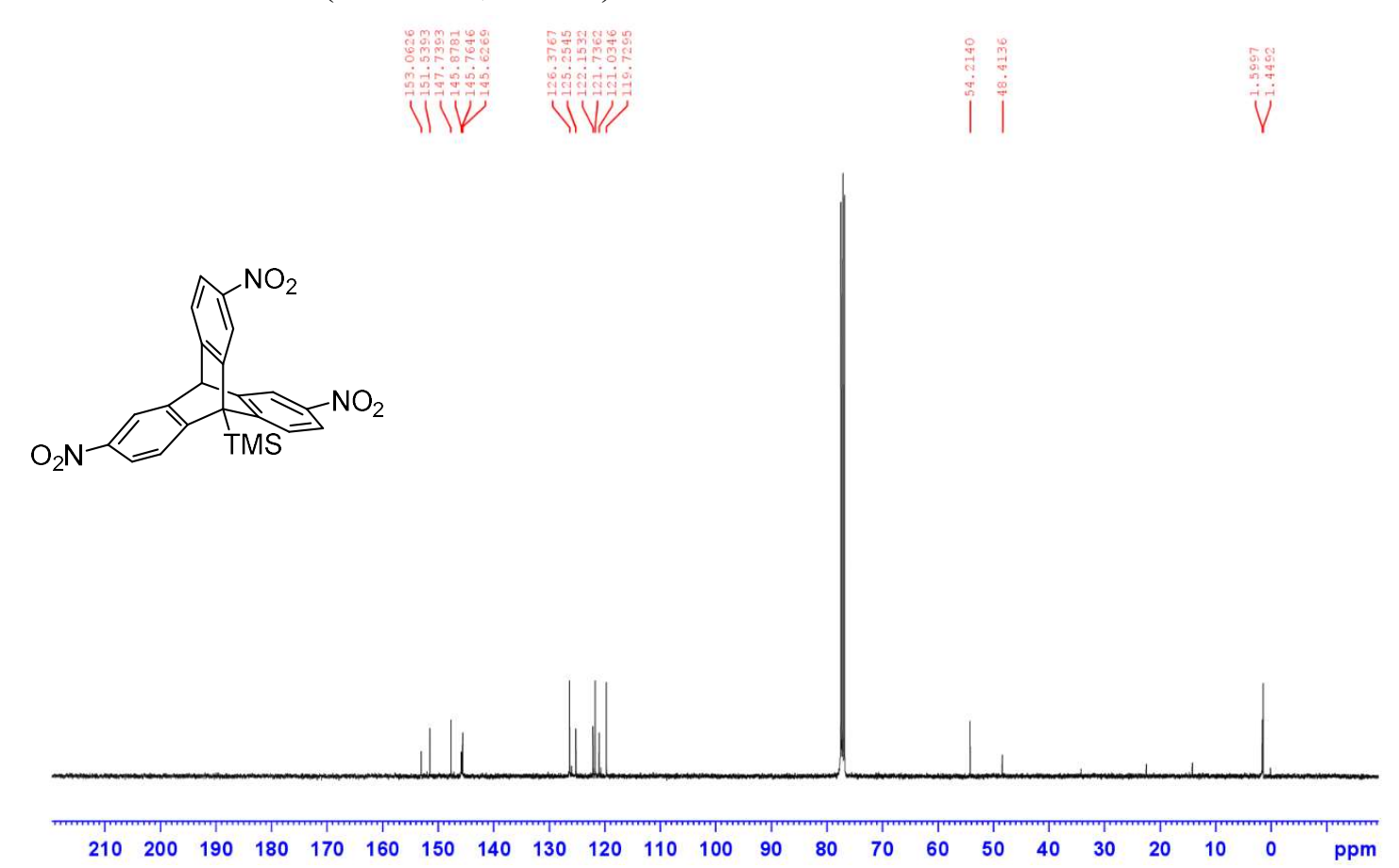

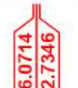

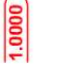


HSQC of isomer $\mathbf{K}\left(\mathrm{CDCl}_{3}\right)$

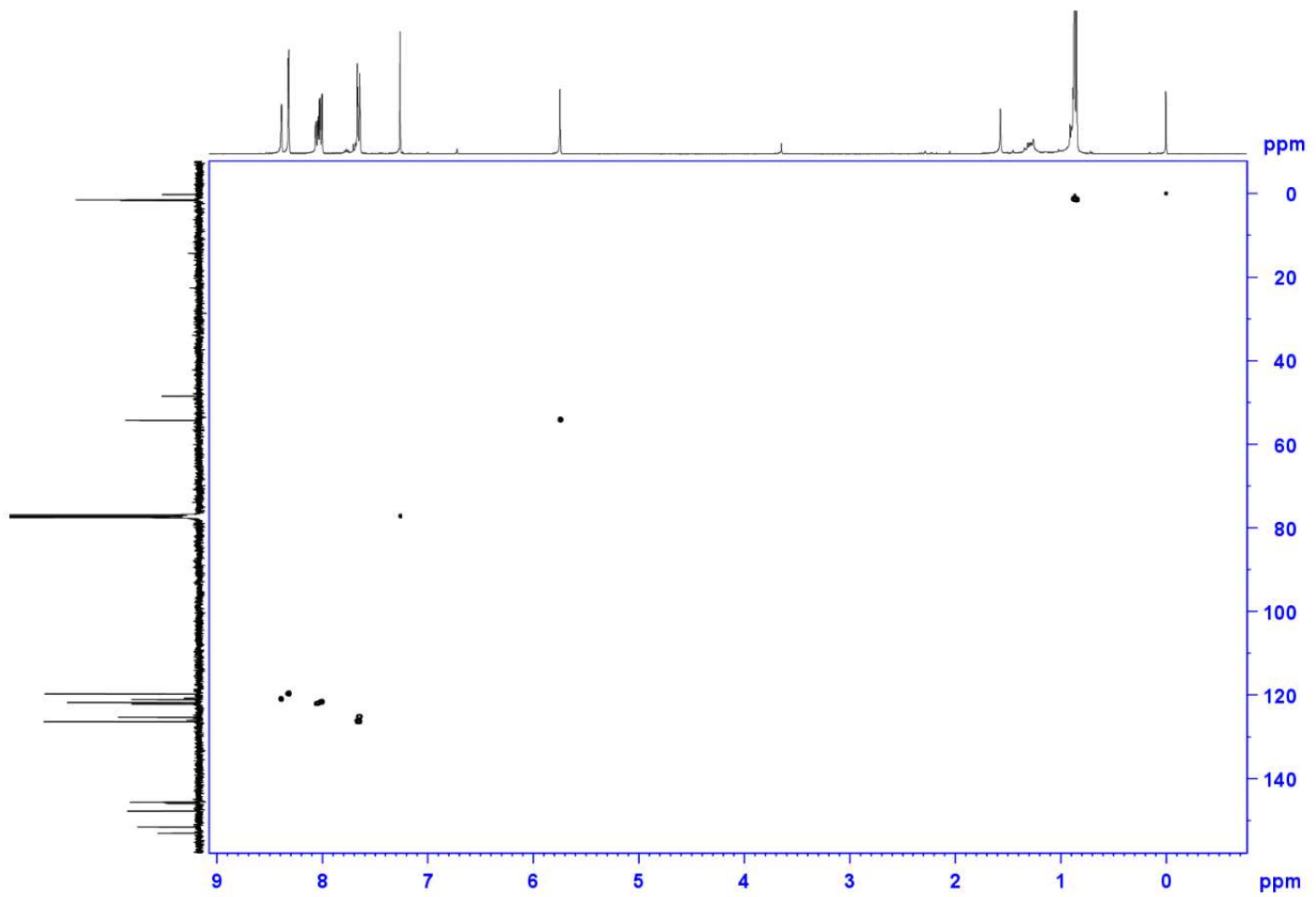

$\mathrm{HMBC}$ of isomer $\mathbf{K}\left(\mathrm{CDCl}_{3}\right)$

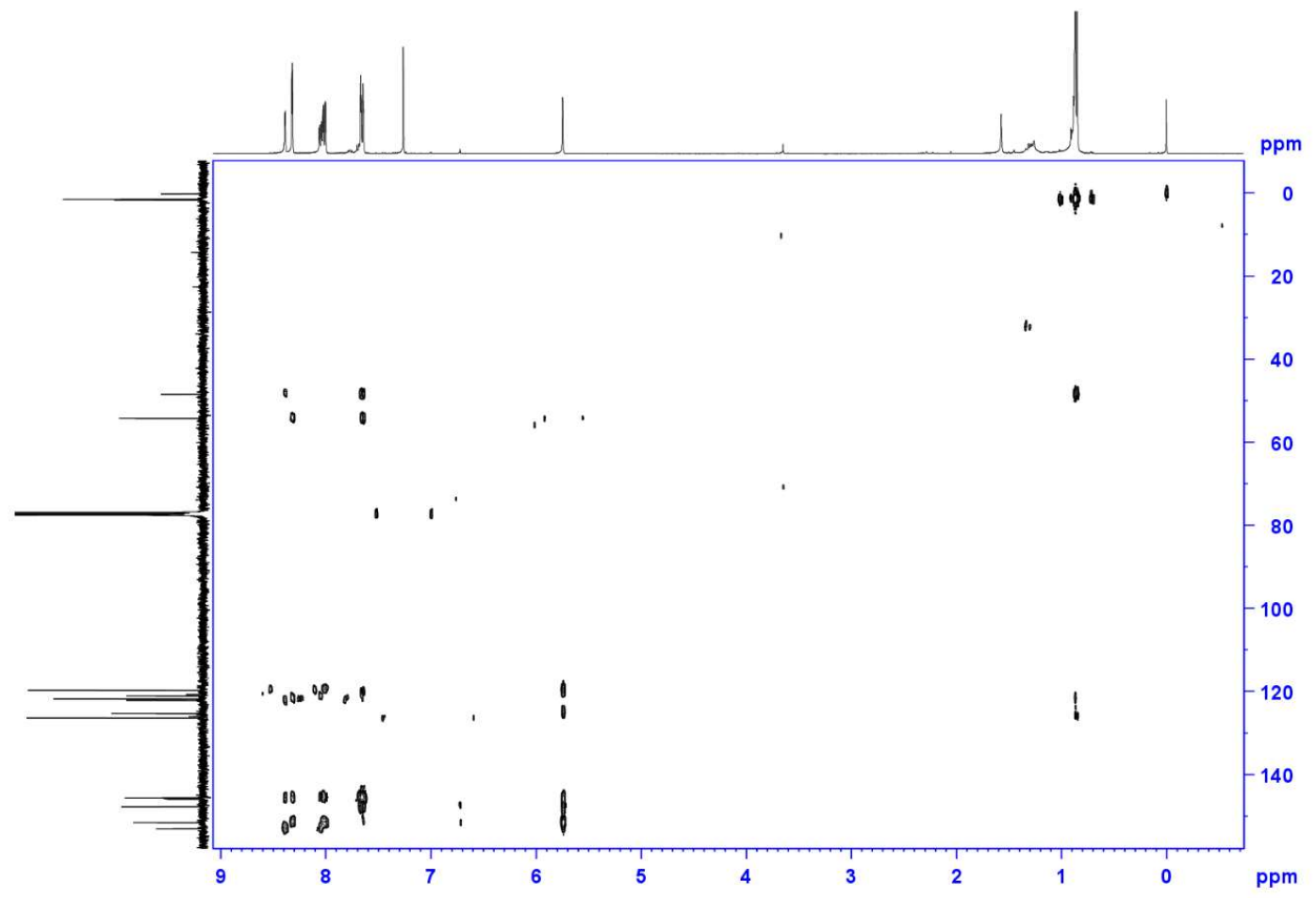


${ }^{1} \mathrm{H}$ NMR of isomer $\mathbf{O}\left(400 \mathrm{MHz}, \mathrm{CDCl}_{3}\right)$

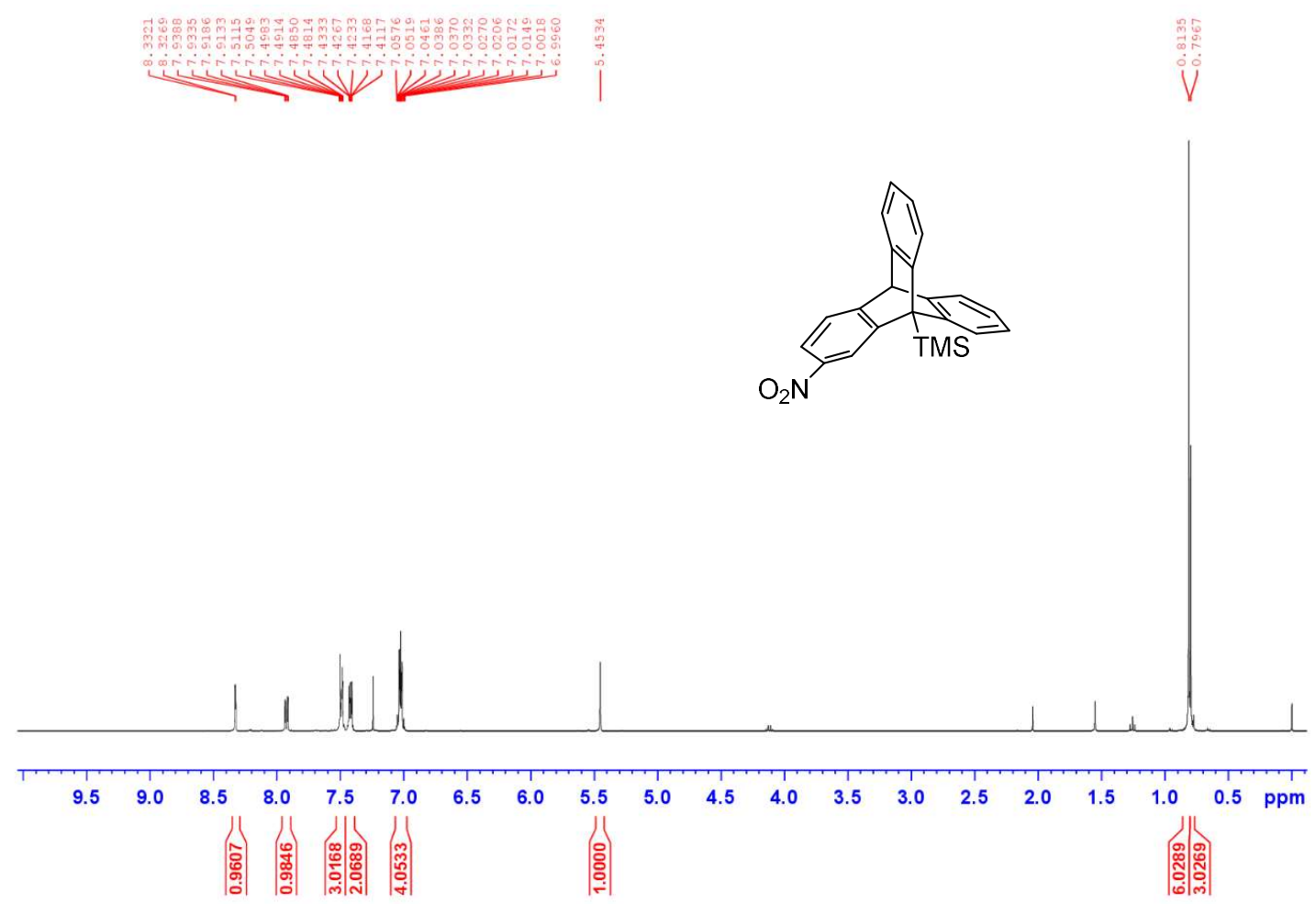

HMBC of isomer $\mathbf{O}\left(\mathrm{CDCl}_{3}\right)$

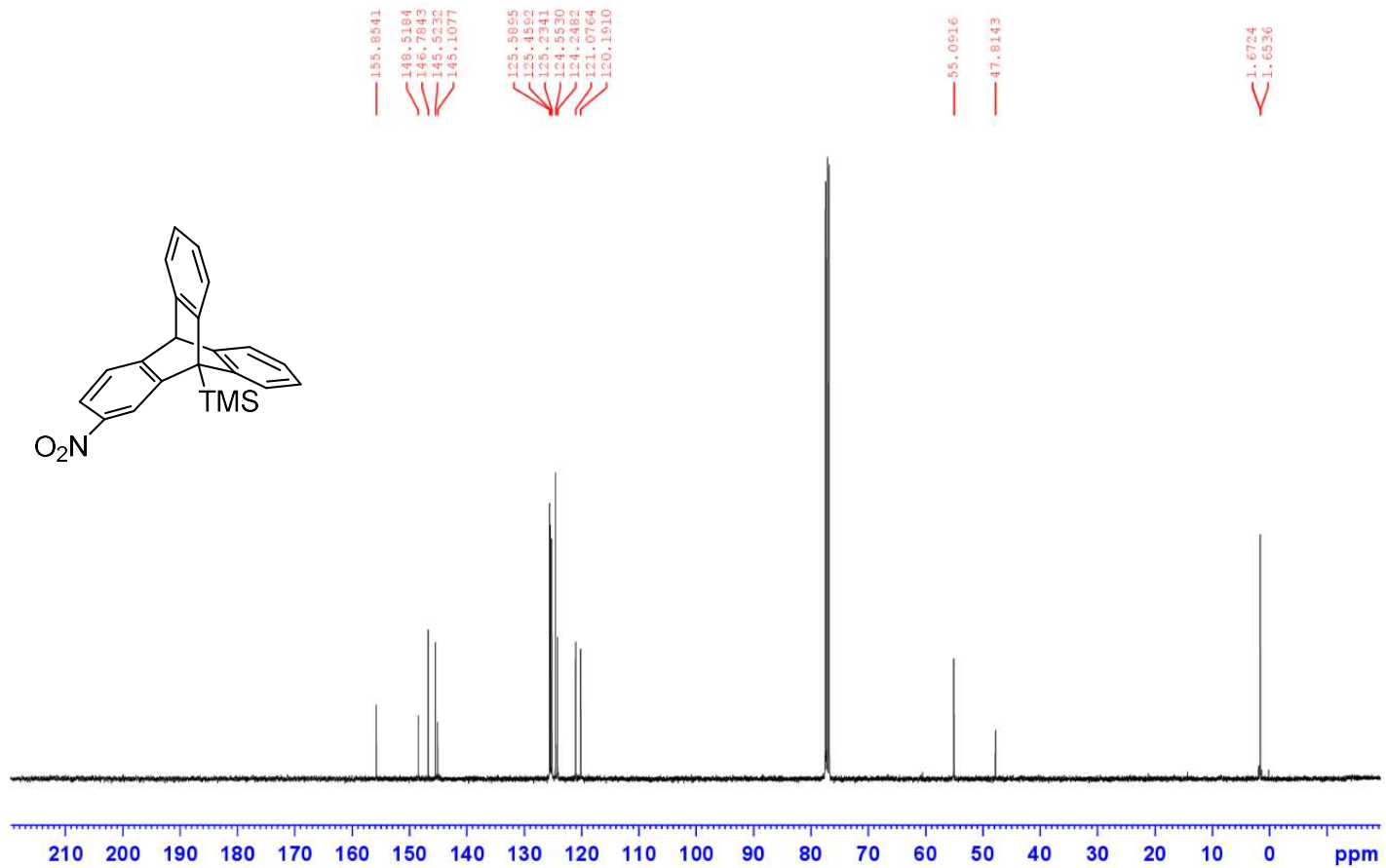


HSQC of isomer $\mathbf{O}\left(\mathrm{CDCl}_{3}\right)$

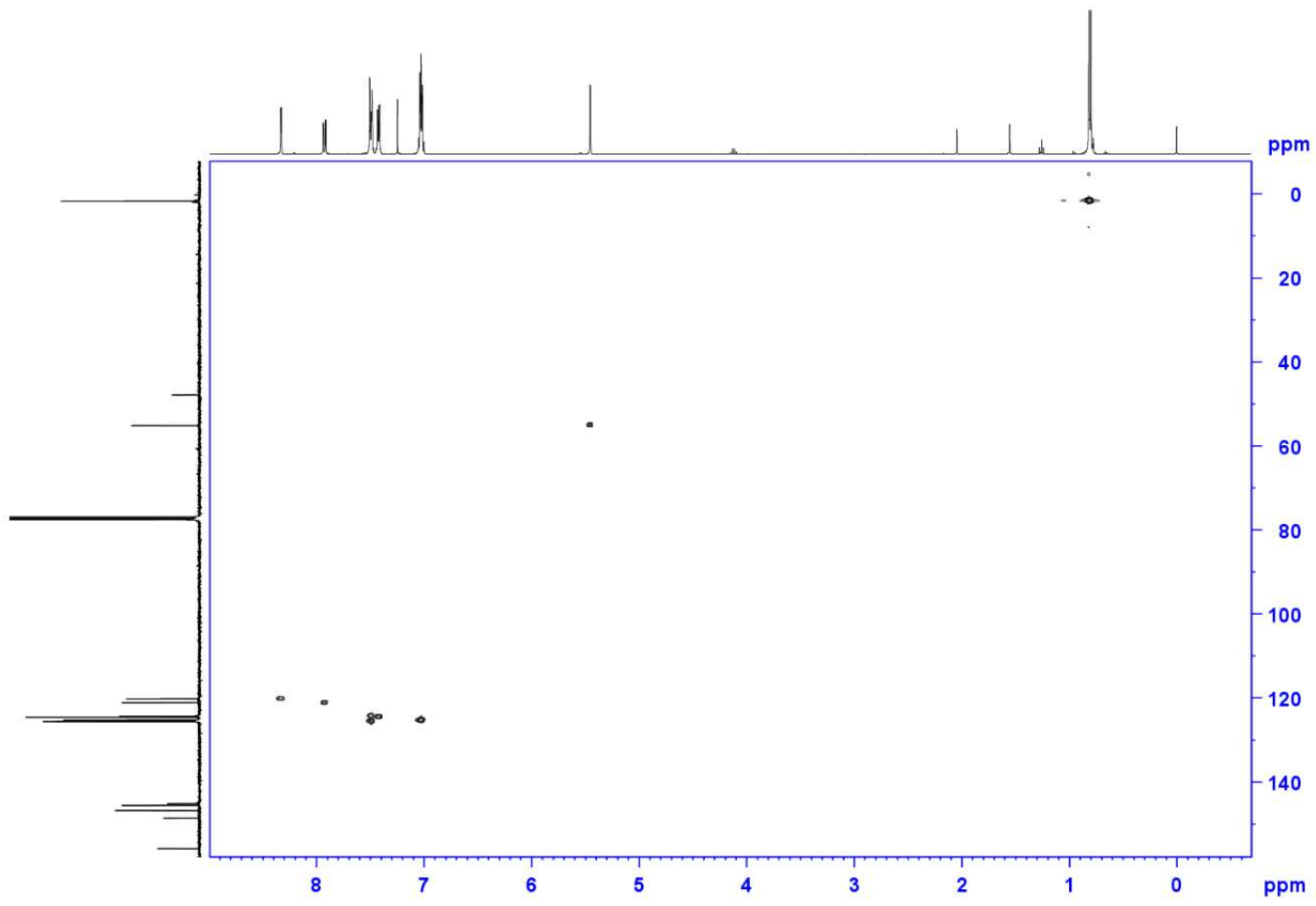

$\mathrm{HMBC}$ of isomer $\mathbf{O}\left(\mathrm{CDCl}_{3}\right)$

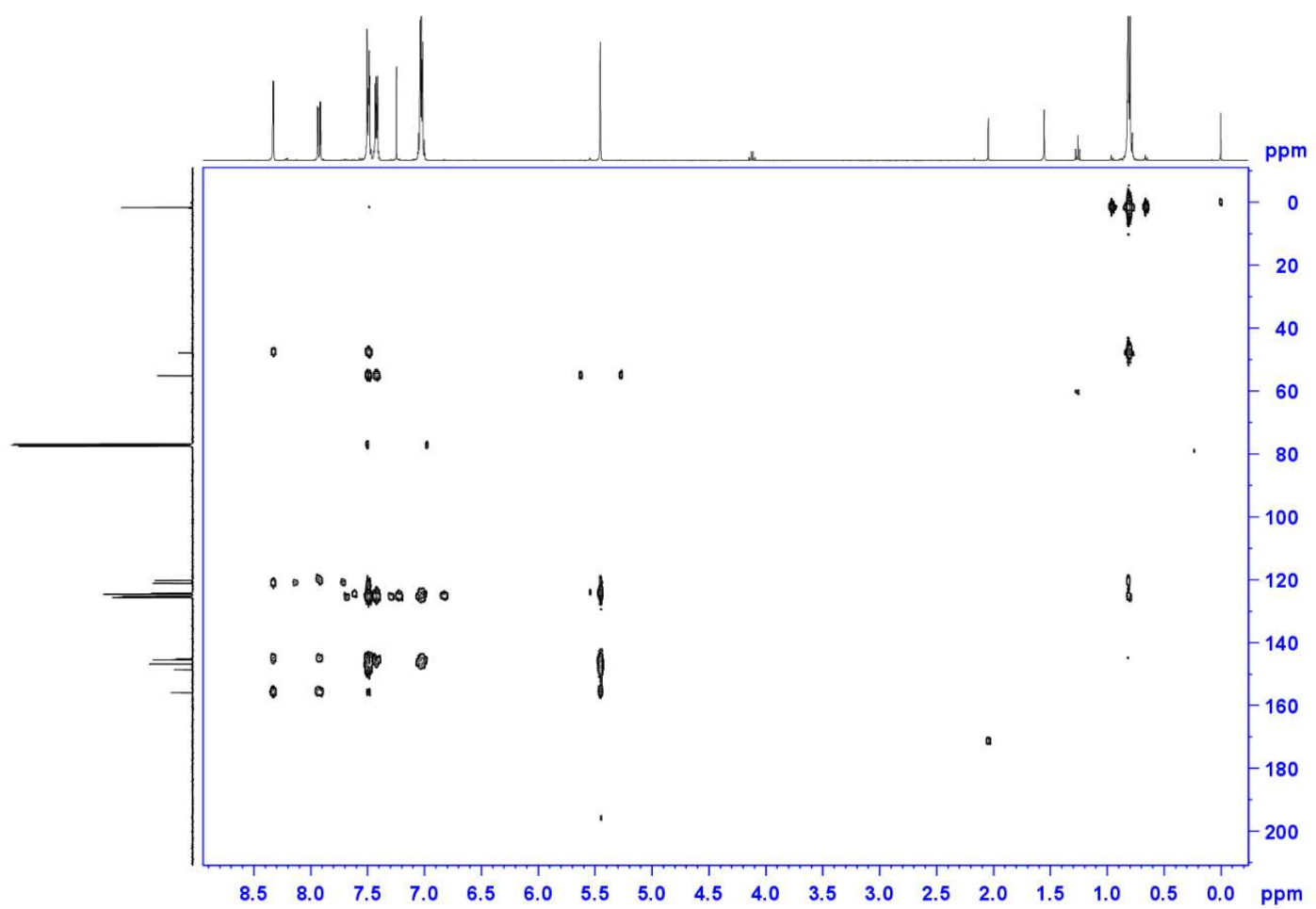


${ }^{1} \mathrm{H}$ NMR of isomer $\mathbf{P}\left(400 \mathrm{MHz}, \mathrm{CDCl}_{3}\right)$
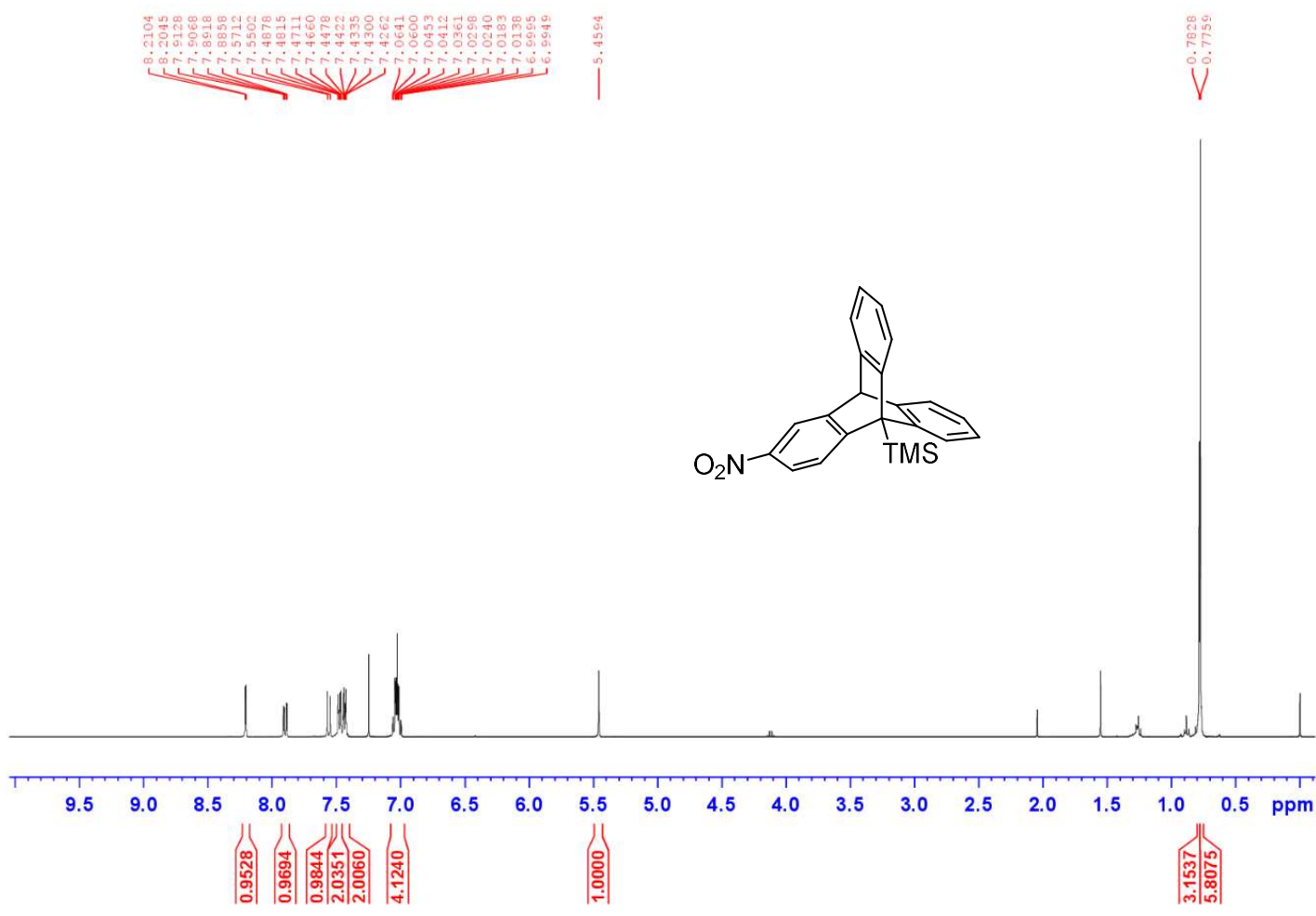

${ }^{13} \mathrm{C} \mathrm{NMR}$ of isomer $\mathbf{P}\left(100 \mathrm{MHz}, \mathrm{CDCl}_{3}\right)$
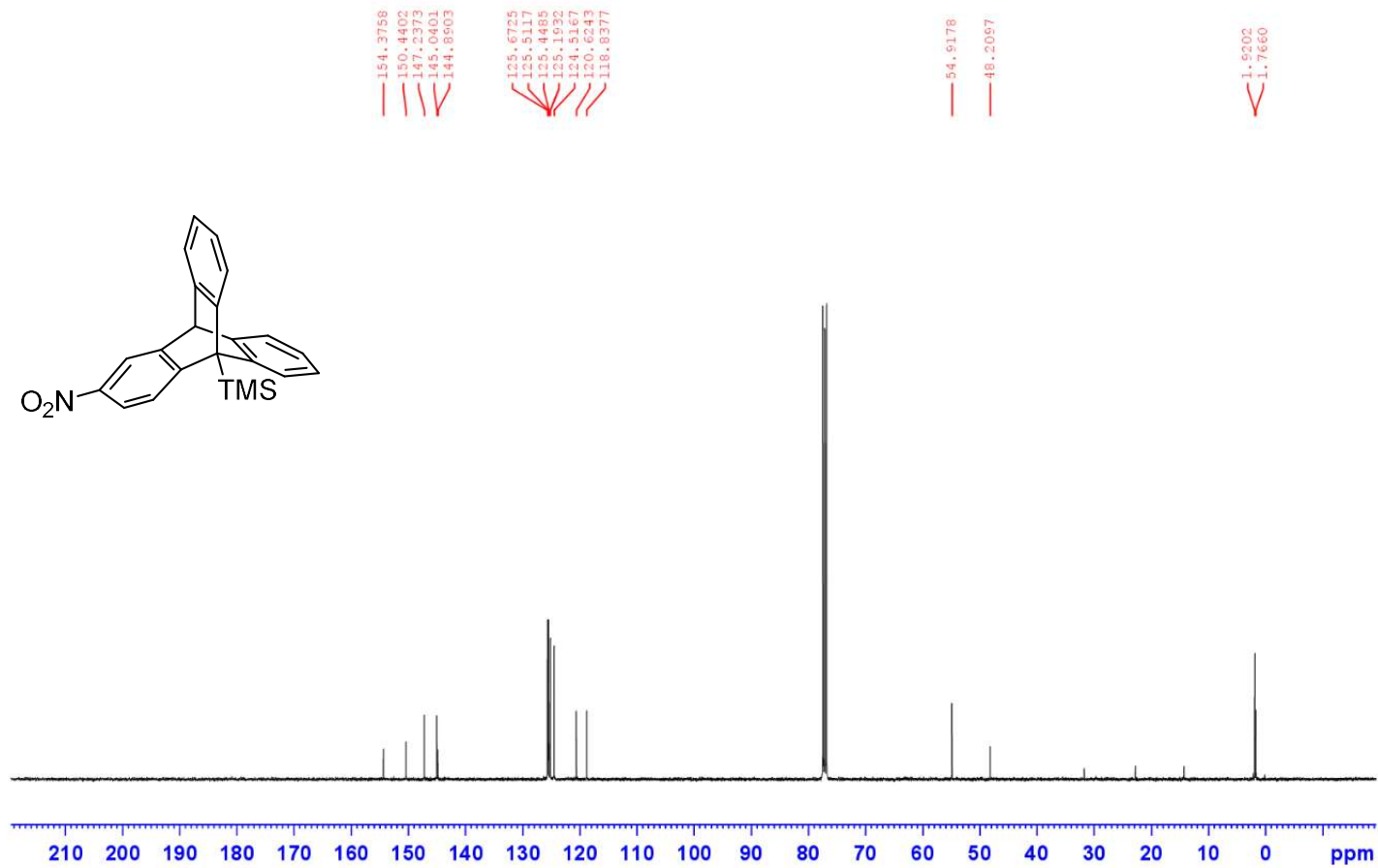
HSQC of isomer $\mathbf{P}\left(\mathrm{CDCl}_{3}\right)$

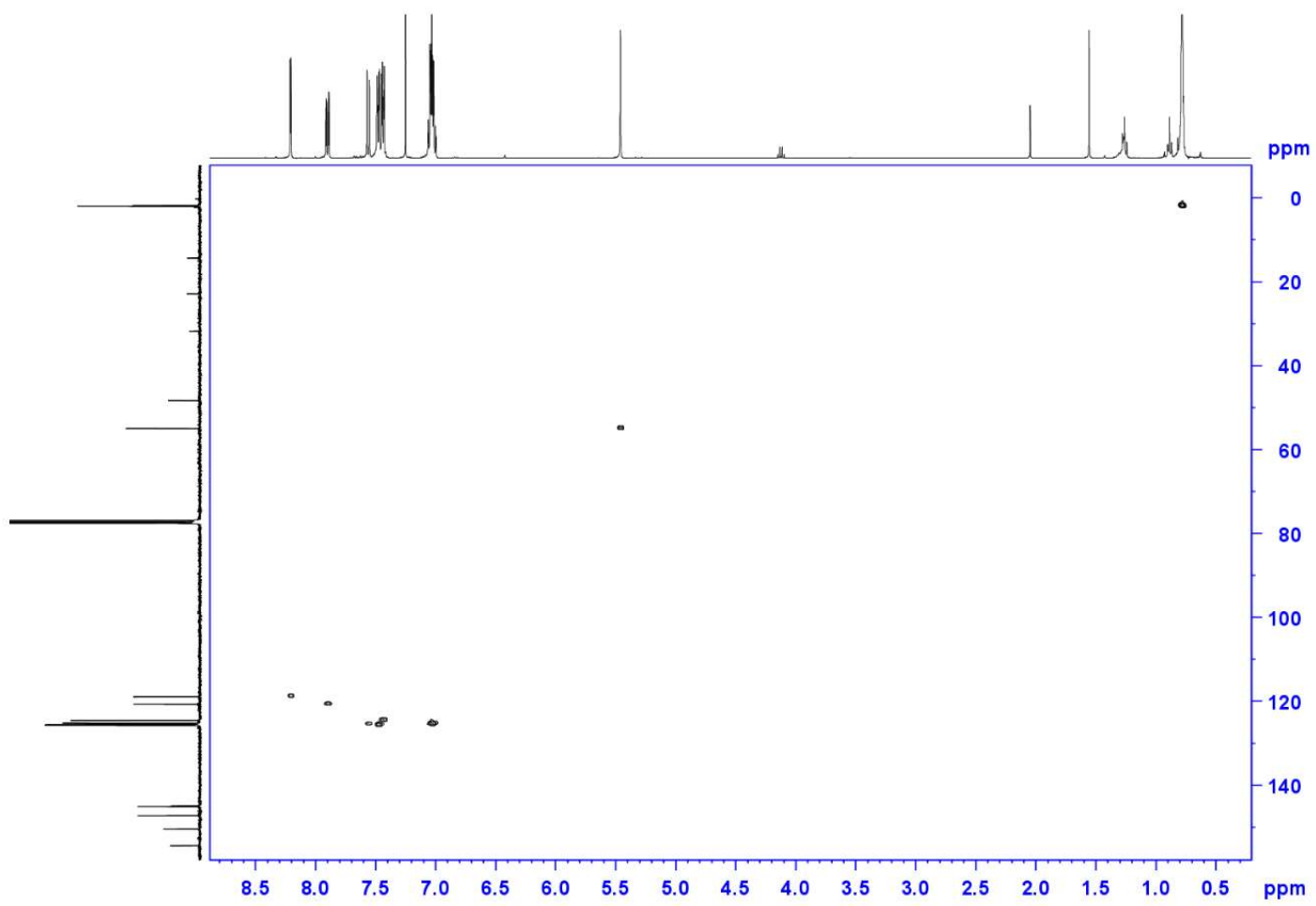

HMBC of isomer $\mathbf{P}\left(\mathrm{CDCl}_{3}\right)$

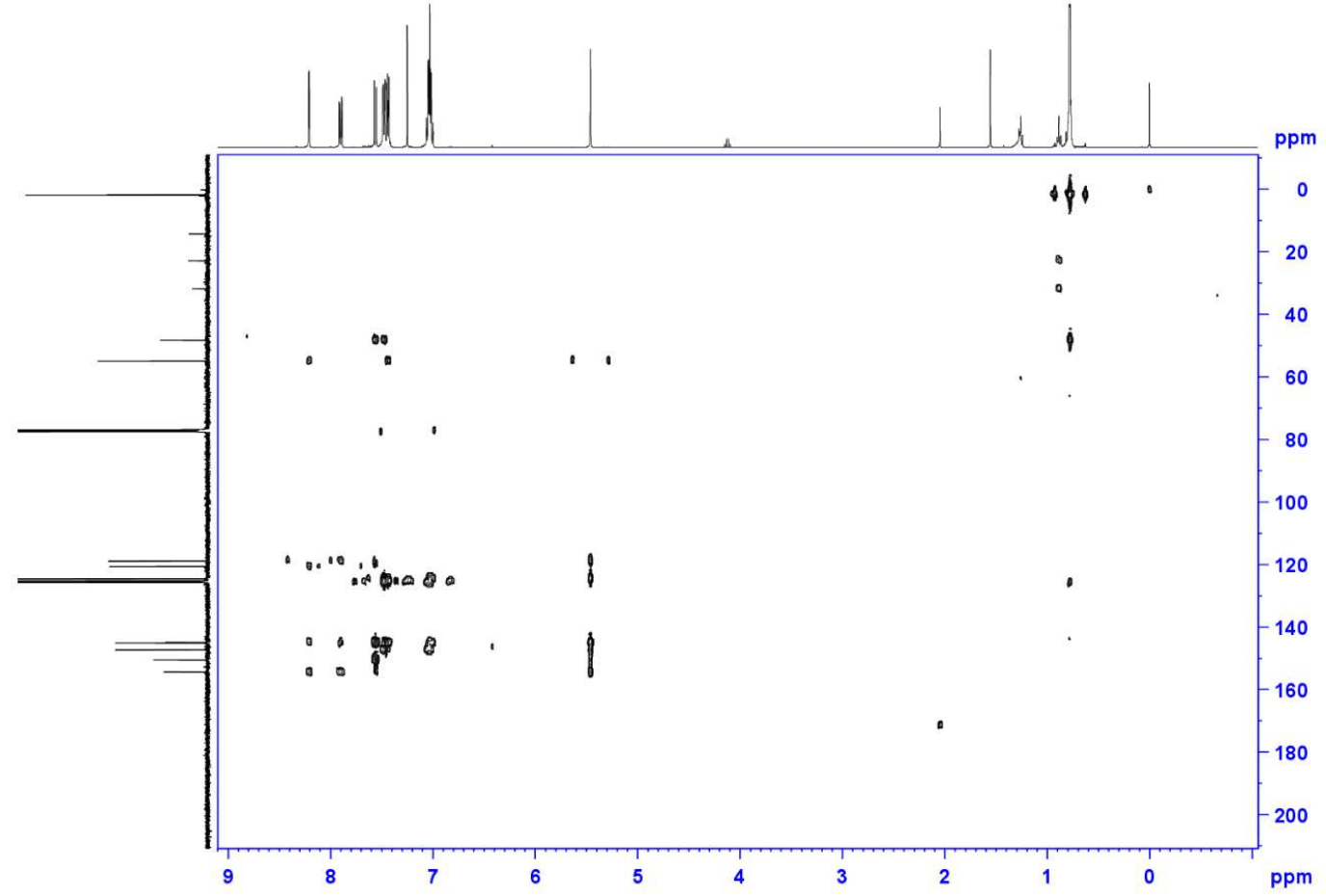




\section{References}

[Supporting references for the main text]

6 (e) Zhang, C.; Liu, Y.; Xiong, X.-Q.; Peng, L.-H.; Gan, L.; Chen, C.-F.; Xu, H.-B. Three-dimensional nanographene based on triptycene: Synthesis and its application in fluorescence imaging. Org. Lett. 2012, 14, 5912-5915. (f) Zhang, C.; Wang, J.-J.; Liu, Y.; Ma, H.; Yang, X.-L.; Xu, H.-B. Main-chain organometallic microporous polymers based on triptycene: Synthesis and catalytic application in the suzuki-miyaura coupling reaction. Chem. - Eur. J. 2013, 19, 5004-5008. (g) Chakraborty, S.; Mondal, S.; Bhowmick, S.; Ma, J.; Tan, H.; Neogi, S.; Das, N. Triptycene based organometallic complexes: A new class of acceptor synthons for supramolecular ensembles. Dalton Trans. 2014, 43, 13270-13277. (h) Synthesis, characterization and DNA interaction studies of new triptycene derivatives. Chakraborty, S.; Mondal, S.; Kumari, R.; Bhowmick, S.; Das, P.; Das, N. Beilstein J. Org. Chem. 2014, 10, 1290-1298. (i) Chakraborty, S.; Mondal, S.; Das, N. Triptycene based chemosensors for selective detection of mercury(II)/iron(III) in aqueous medium. Inorg. Chim. Acta 2014, 413, 214-220. (j) Zhu, P.-C.; Liu, Y.; Peng, L.-H.; Zhang, C. Synthesis and structures of Hexa-peri-hexabenzocoronene-based triptycenes. Tetrahedron Lett. 2014, 55, 521-524. (k) Moylan, C.; Rogers, L.; Shaker, Y. M.; Davis, M.; Eckhardt, H.-G.; Eckert, R.; Ryan, A. A.; Senge, M. O. Preparation of Tri- and Hexasubstituted Triptycene Synthons by Transition Metal Catalyzed Cross-Coupling Reactions for Post-Modifications. Eur. J. Org. Chem. 2016, 2016, 185-195. (1) Huang, X.; Zhao, Y.-C.; Han, B.-H. Supramolecular organic network assembled from quadruple hydrogen-bonding motifs. Chem. Commun. 2016, 52, 6597-6600. (m) Emandi, G.; Browne, M. P.; Lyons, M. E.; Prior, C.; Senge, M. O. Triptycene scaffolds: Synthesis and properties of triptycene-derived Schiff base compounds for the selective and sensitive detection of $\mathrm{CN}^{-}$and $\mathrm{Cu}^{2+}$. Tetrahedron 2017, 73, 2956-2965. (n) Liu, X.; Cai, Y.; Huang, X.; Zhang, R.; Sun, X. A perylene diimide electron acceptor with a triptycene core for organic solar cells. J. Mat. Chem. C 2017, 5, 3188-3194. (o) Chakraborty, S.; Endres, K. J.; Bera, R.; Wojtas, L.; Moorefield, C. N.; Saunders, M. J.; Das, N.; Wesdemiotis, C.; Newkome, G. R. Concentration dependent supramolecular interconversions of triptycene-based cubic, prismatic, and tetrahedral structures. Dalton Trans. 2018, 47, 14189-14194 (p) Jiang, N.; Yuan, Z.; Li, T.; Zhu, Y.; Chen, Y.-S.; Lin, L.; Zhang, J.; Chan, Y.-T.; Wang, J. Synthesis and Characterization of Ferrocene Based Hemicages. J. Org. Chem. 2018, 83, 4824-4830. (q) Wang, L.; Li, W.; Wang, Z.; Luo, Q. Triptycene-scaffolded tetraphenylethylenes with irregular temperaturedependence AIE. Tetrahedron Lett. 2019, 60, 439-443. (r) Reinhard, D.; Rominger, F.; Mastalerz, M. Desymmetrization Strategy to Achieve Triptycene-Based 3,6-Dimethoxytriphenylenes via Oxidative Cyclodehydrogenation. Eur. J. Org. Chem. 2020, 2020, 6255-6258. (s) Jayakumar, J.; Wu, W.-L.; Chang, C.-L.; Han, T.-Y.; Ting, L.-Y.; Yeh, C.-M.; Hung, H.-W.; Chou, H.-H. Highly thermal stable electron-transporting materials using triptycene derivatives for OLEDs. Org. Electron. 2021, 88, 106013. 
[References for the Supporting Information]

${ }^{1}$ Nishii, Y.; Ikeda, M.; Hayashi, Y.; Kawauchi, S.; Miura, M. Triptycenyl Sulfide: A Practical and Active Catalyst for Electrophilic Aromatic Halogenation Using N-Halosuccinimides. J. Am. Chem. Soc. 2020, 142 , 1621-1629.

${ }^{2}$ D. J. Murray, D. D. Patterson, P. Payamyar, R. Bhola, W. Song, M. Lackinger, A. D. Schlüter, B. T. King. Large Area Synthesis of a Nanoporous Two-Dimensional Polymer at the Air/Water Interface $J$. Am. Chem. Soc. 2015, 137, 3450-3453.

${ }^{3}$ Calvo, R.; Zhang, K.; Passera, A.; Katayev, D. Facile access to nitroarenes and nitroheteroarenes using $N$ nitrosaccharin. Nat. Commun. 2019, 10, 3410.

${ }^{4}$ Parish, R. C.; Stock. L. M. 9-t-Butylanthracene. J. Org. Chem. 1966, 31, 4265-4267.

${ }^{5}$ Seki, S.; Morinaga T.; Kikuchi H.; Mitsuhashi T.; Yamamoto G.; Oki M. Reactivities of Stable Rotamers. VI. Manifestation of Differential Reactivities of the Methyls in a $t$-Butyl Group in Radical Halogenations. Bull. Chem. Soc. Jpn. 1981, 54, 1465-1469.

${ }^{6}$ Zhang, C.; Chen, C.-F. Synthesis and Structure of 2,6,14- and 2,7,14-Trisubstituted Triptycene Derivatives. $J$. Org. Chem. 2006, 71, 6626-6629.

${ }^{7}$ Chakraborty, S.; Mondal, S.; Kumari, R.; Bhowmick, S.; Das, P.; Das, N. Synthesis, characterization and DNA interaction studies of new triptycene derivatives. Beilstein J. Org. Chem. 2014, 10, 1290-1298.

${ }^{8}$ Sheldrick, G. M. A short history of SHELX. Acta Crystallogr. Sect. A 2008, 64, 112-122.

${ }^{9}$ Farrugia, L. J. WinGX and ORTEP for Windows: an update. J. Appl. Cryst. 2012, 45, 849-854.

${ }^{10}$ Frisch, M. J.; Trucks, G. W.; Schlegel, H. B.; Scuseria, G. E.; Robb, M. A.; Cheeseman, J. R.; Scalmani, G.; Barone, V.; Petersson, G. A.; Nakatsuji, H.; Li, X.; Caricato, M.; Marenich, A. V.; Bloino, J.; Janesko, B. G.; Gomperts, R.; Mennucci, B.; Hratchian, H. P.; Ortiz, J. V.; Izmaylov, A. F.; Sonnenberg, J. L.; Williams-Young, D.; Ding, F.; Lipparini, F.; Egidi, F.; Goings, J.; Peng, B.; Petrone, A.; Henderson, T.; Ranasinghe, D.; Zakrzewski, V. G.; Gao, J.; Rega, N.; Zheng, G.; Liang, W.; Hada, M.; Ehara, M.; Toyota, K.; Fukuda, R.; Hasegawa, J.; Ishida, M.; Nakajima, T.; Honda, Y.; Kitao, O.; Nakai, H.; Vreven, T.; Throssell, K.; Montgomery, J. A., Jr.; Peralta, J. E.; Ogliaro, F.; Bearpark, M. J.; Heyd, J. J.; Brothers, E. N.; Kudin, K. N.; Staroverov, V. N.; Keith, T. A.; Kobayashi, R.; Normand, J.; Raghavachari, K.; Rendell, A. P.; Burant, J. C.; Iyengar, S. S.; Tomasi, J.; Cossi, M.; Millam, J. M.; Klene, M.; Adamo, C.; Cammi, R.; Ochterski, J. W.; Martin, R. L.; Morokuma, K.; Farkas, O.; Foresman, J. B.; Fox, D. J. Gaussian, Inc., Wallingford CT, 2016. 